\title{
Contact Geometry of Hyperbolic Equations of Generic Type
}

Dennis THE

McGill University, 805 Sherbrooke Street West, Montreal, QC, H3A 2K6, Canada

E-mail: dthe@math.mcgill.ca

Received April 10, 2008, in final form August 11, 2008; Published online August 19, 2008

Original article is available at http://www.emis.de/journals/SIGMA/2008/058/

\begin{abstract}
We study the contact geometry of scalar second order hyperbolic equations in the plane of generic type. Following a derivation of parametrized contact-invariants to distinguish Monge-Ampère (class 6-6), Goursat (class 6-7) and generic (class 7-7) hyperbolic equations, we use Cartan's equivalence method to study the generic case. An intriguing feature of this class of equations is that every generic hyperbolic equation admits at most a nine-dimensional contact symmetry algebra. The nine-dimensional bound is sharp: normal forms for the contact-equivalence classes of these maximally symmetric generic hyperbolic equations are derived and explicit symmetry algebras are presented. Moreover, these maximally symmetric equations are Darboux integrable. An enumeration of several submaximally symmetric (eight and seven-dimensional) generic hyperbolic structures is also given.
\end{abstract}

Key words: contact geometry; partial differential equations; hyperbolic; generic; nonlinear

2000 Mathematics Subject Classification: 35A30; 35L70; 58J70

\section{Introduction}

The purpose of this paper is to give a reasonably self-contained account of some key geometric features of a class of (nonlinear) scalar second order hyperbolic partial differential equations (PDE) in the plane (i.e. in two independent variables) that has received surprisingly very little attention in the literature, namely hyperbolic PDE of generic (also known as class 7-7) type. Even this terminology is not well-known, and so it deserves some clarification.

In the geometric study of differential equations, there is a natural notion of equivalence associated with the pseudo-group of local point transformations, i.e. local diffeomorphisms which mix the independent and dependent variables. Another natural (but coarser) notion is to define equivalence up to the larger pseudo-group of local contact transformations and one of the principal goals of the geometric theory is to find invariants to distinguish different contact-equivalence classes. Restricting now (and for the remainder of this paper) to scalar second order PDE in the plane, we have that given certain nondegeneracy conditions (i.e. one can locally solve the equation for one of the highest derivatives), there is a contact-invariant trichotomy into equations of elliptic, parabolic and hyperbolic type. In the quasi-linear case, invariance of these classes under point transformations appears in [7]. (Inequivalent normal forms are derived in each case.) An elegant geometric proof of invariance under contact transformations in the general case was given by R.B. Gardner [9]. In the hyperbolic case, there exist two characteristic subsystems which give rise to a finer contact-invariant trichotomy into equations of Monge-Ampère (class 6-6), Goursat (class 6-7), and generic (class 7-7) type. While this was known to Vranceanu and almost certainly to E. Cartan and Lie, a modern exposition of these ideas first appeared in [11]. To keep our exposition as self-contained as possible, we include

\footnotetext{
*This paper is a contribution to the Special Issue "Élie Cartan and Differential Geometry". The full collection is available at http://www.emis.de/journals/SIGMA/Cartan.html
} 
the details of these classifications in this paper. For hyperbolic equations given in the form $z_{x x}=f\left(x, y, z, z_{x}, z_{y}, z_{x y}, z_{y y}\right)$, the (relative) invariants characterizing the three types of hyperbolic equations were calculated parametrically by Vranceanu (c.f. the $B, C$ invariants in [26]). For a general equation $F\left(x, y, z, z_{x}, z_{y}, z_{x x}, z_{x y}, z_{y y}\right)=0$, these invariants appeared in Chapter 9 of Juráś' thesis in his characterization of the Monge-Ampère class (c.f. $M_{\sigma}, M_{\tau}$ in [13]). Our derivation of these invariants (labelled $I_{1}, I_{2}$ in this article) is quite different and the novelty in our exposition (see Theorem 3.3) is obtaining simpler expressions expressed in terms of certain determinants. Moreover, we use these invariants to give general examples of hyperbolic equations of Goursat and generic type (see Table 3).

Hyperbolic equations of Monge-Ampère type have been well-studied in the literature from a geometric point of view (e.g. see [16, 17, 19, 4, 5, 15, 2, 14, 18] and references therein). This class of equations includes the Monge-Ampère, wave, Liouville, Klein-Gordon and general $f$-Gordon equations. At the present time and to the best of our knowledge, there exists only one paper in the literature that has been devoted to the study of hyperbolic equations of generic type. This paper, "La géométrisation des équations aux dérivées partielles du second ordre" [25], was published by Vranceanu in 1937. Despite its appearance over 70 years ago, and much attention having been focused on applications of Cartan's equivalence method in the geometric theory of PDE, very few references to [25] exist. Currently, the paper does not appear on MathSciNet; the only reference to it by internet search engines appears on Zentralblatt Math.

In [25], Vranceanu uses the exterior calculus and Cartan's method of equivalence to study generic hyperbolic equations. One of the most intriguing results of the paper is that all equations of generic type admit at most a nine-dimensional local Lie group of (contact) symmetries. This is in stark contrast to the Monge-Ampère class, where the wave equation is well-known to admit an infinite-dimensional symmetry group. Vranceanu is able to isolate the corresponding maximally symmetric structure equations as well as some submaximally symmetric structures. Furthermore, he is able to integrate these abstract structure equations to obtain an explicit parametrization of the corresponding coframe, leading to normal forms for the contactequivalence classes. As any practitioner of the Cartan equivalence method can probably attest, this is an impressive computational feat. Nevertheless, as in the style of Cartan's writings, Vranceanu's arguments are at times difficult to decipher, hypotheses are not clearly stated or are difficult to discern amidst the quite lengthy calculations, and some of his results are not quite correct. In this paper, we reexamine, clarify, and sharpen some of Vranceanu's results with the perspective of our modern understanding of the geometric theory of differential equations through exterior differential systems and Cartan's equivalence method. The hope is that this exposition will provide a clearer understanding of the geometry of this class of equations for a contemporary audience.

In Section 2 we recall the contact-invariant classification of second order scalar PDE into elliptic, parabolic, and hyperbolic classes based on invariants of a (conformal class of a) symmetric bilinear form, and define the $M_{1}$ and $M_{2}$ characteristics in the hyperbolic case. This leads to a preliminary set of structure equations for hyperbolic equations. In Section 3, the structure equations are further tightened, and using them we show how the class of $M_{1}$ and $M_{2}$ leads to the finer classification into equations of Monge-Ampère, Goursat, and generic types. In Theorem 3.3, these subclasses of hyperbolic equations are characterized by means of the relative invariants $I_{1}, I_{2}$. We then restrict to the generic case and derive the generic hyperbolic structure equations. We note that in Vranceanu's derivation of the generic hyperbolic structure equations (c.f. (3.2) in this paper), the $\epsilon=\operatorname{sgn}\left(I_{1} I_{2}\right)= \pm 1$ contact invariant was overlooked. This carries through to the normal forms for the contact-equivalence classes. Section 4 formulates the equivalence problem for generic hyperbolic equations and recalls some facts from Cartan's theory of $G$-structures applied to our situation. The structure group that we consider here is strictly larger than Vranceanu's, differing by certain discrete components. These naturally arise when 
considering automorphisms which interchange the $M_{1}$ and $M_{2}$ characteristics. Both Vranceanu and Gardner-Kamran consider only automorphisms which preserve each of $M_{1}$ and $M_{2}$. The nine-dimensional bound on the symmetry group of any generic hyperbolic equation is established in Section 5.

In Section 6, we give a clear enumeration of several generic hyperbolic structures which result from Vranceanu's reduction of the structure equations. These include the aforementioned maximally symmetric (nine-dimensional) structure equations as well as some new submaximally symmetric (eight and seven-dimensional) structures including some with nonconstant torsion. (Vranceanu derived two eight-dimensional structures with constant torsion in addition to the maximally symmetric structures.) Finally, Section 7 gives a detailed account of the maximally symmetric case. Integration of the abstract maximally symmetric structure equations leads to the contact-equivalence classes of maximally symmetric generic hyperbolic PDE being parametrized by $(\epsilon, a) \in\{ \pm 1\} \times(0,1]$, with normal forms given by

$$
\begin{array}{ll}
(\epsilon, a)=(1,1): & 3 z_{x x}\left(z_{y y}\right)^{3}+1=0, \\
(\epsilon, a) \neq(1,1): & (\epsilon+a)^{2}\left(2 z_{x y}-\left(z_{y y}\right)^{2}\right)^{3}+\epsilon a\left(3 z_{x x}-6 z_{x y} z_{y y}+2\left(z_{y y}\right)^{3}\right)^{2}=0 .
\end{array}
$$

The isomorphism type of the symmetry algebra for the second equation is independent of $(\epsilon, a)$ and is non-isomorphic to the symmetry algebra of the first equation. Thus, there are precisely two non-isomorphic (abstract) symmetry algebras that arise for maximally symmetric generic hyperbolic equations. These equations are further distinguished in a contact-invariant way using a contact invariant $\Delta_{1}$ and a relative contact invariant $\Delta_{2}$. Both equations satisfy $\Delta_{1}=0$, but the former satisfies $\Delta_{2}=0$ while the latter satisfies $\Delta_{2} \neq 0$.

Let us point out two additional points of discrepancy with Vranceanu's calculations: (1) the restriction of the range of the parameter $a$ to $(0,1]$, and $(2)$ a missing factor of 2 for the $z_{x y} z_{y y}$ term in the second equation above. The first point is a consequence of the aforementioned larger structure group used in our formulation of the equivalence problem. The additional discrete factors lead to identifications of different parameter values. The second point was minor and the error was only introduced by Vranceanu in the last step of his derivation. To give added justification to the calculation of the normal forms above, we give explicitly the nine-dimensional symmetry algebras for the normal forms listed above. Both equations admit the symmetries

$$
\begin{aligned}
& X_{1}=\frac{\partial}{\partial x}, \quad X_{2}=\frac{\partial}{\partial y}, \quad X_{3}=\frac{\partial}{\partial z}, \quad X_{4}=x \frac{\partial}{\partial z}, \quad X_{5}=y \frac{\partial}{\partial z}, \\
& X_{6}=x \frac{\partial}{\partial x}+y \frac{\partial}{\partial y}+2 z \frac{\partial}{\partial z} .
\end{aligned}
$$

The former admits the additional symmetries

$$
X_{7}=x y \frac{\partial}{\partial z}, \quad X_{8}=2 y \frac{\partial}{\partial y}+3 z \frac{\partial}{\partial z}, \quad X_{9}=x^{2} \frac{\partial}{\partial x}+x z \frac{\partial}{\partial z},
$$

while the latter admits the additional symmetries

$$
X_{7}=y \frac{\partial}{\partial y}+3 z \frac{\partial}{\partial z}, \quad X_{8}=x \frac{\partial}{\partial y}-\frac{1}{2} y^{2} \frac{\partial}{\partial z}, \quad X_{9}=x^{2} \frac{\partial}{\partial x}+x y \frac{\partial}{\partial y}+\left(x z-\frac{1}{6} y^{3}\right) \frac{\partial}{\partial z} .
$$

The calculation of these symmetries (especially in the latter case) is in general a nontrivial task considering the complexity of the equation.

Numerous appendices provide the details of the proofs of the main statements in the body of this article.

All considerations in this paper are local, and we will work in the smooth category. We use the Einstein summation convention throughout. We will make the convention of using 
braces enclosing 1-forms to denote the corresponding submodule generated by those 1-forms. In general, we will abuse notation and not distinguish between a submodule of 1 -forms and its corresponding algebraic ideal (i.e. with respect to the wedge product) in the exterior algebra. This is useful when stating structure equations, e.g. $d \omega^{1} \equiv 0 \bmod I_{F}$.

\section{Contact-equivalence of PDE}

Consider a scalar second order PDE

$$
F\left(x, y, z, \frac{\partial z}{\partial x}, \frac{\partial z}{\partial y}, \frac{\partial^{2} z}{\partial x^{2}}, \frac{\partial^{2} z}{\partial x \partial y}, \frac{\partial^{2} z}{\partial y^{2}}\right)=0
$$

in two independent variables $x, y$ and one dependent variable $z$. A natural geometrical setting for $(2.1)$ is the space of 2 -jets $J^{2}\left(\mathbb{R}^{2}, \mathbb{R}\right)$ with standard local coordinates $(x, y, z, p, q, r, s, t)$ (Monge coordinates), and the equation above yields a locus

$$
L_{F}=\left\{(x, y, z, p, q, r, s, t) \in J^{2}\left(\mathbb{R}^{2}, \mathbb{R}\right): F(x, y, z, p, q, r, s, t)=0\right\} .
$$

We assume that $L_{F}$ is the image of an open subset $\Sigma_{7} \subset \mathbb{R}^{7}$ under a smooth map $i_{F}: \Sigma_{7} \rightarrow$ $J^{2}\left(\mathbb{R}^{2}, \mathbb{R}\right)$.

Definition 2.1. We will say that $i_{F}$ is a nondegenerate parametrization of the equation $F=0$ if $i_{F}$ has maximal rank and $L_{F}$ is everywhere transverse to the fibers of the natural projection

$$
\pi_{1}^{2}: J^{2}\left(\mathbb{R}^{2}, \mathbb{R}\right) \rightarrow J^{1}\left(\mathbb{R}^{2}, \mathbb{R}\right),
$$

i.e. $\operatorname{im}\left(i_{F *}\right)+\operatorname{ker}\left(\pi_{1 *}^{2}\right)=T J^{2}\left(\mathbb{R}^{2}, \mathbb{R}\right)$.

We will always work with nondegenerate parametrizations in this paper. By the transversality assumption $\left(F_{r}, F_{s}, F_{t}\right) \neq 0$, and so by the implicit function theorem, one can locally solve (2.1) for one of the highest-order derivatives. Since $\operatorname{im}\left(\left(\pi_{1}^{2} \circ i_{F}\right)_{*}\right)=T J^{1}\left(\mathbb{R}^{2}, \mathbb{R}\right)$, then $\left(\pi_{1}^{2} \circ i_{F}\right)^{*}(d x \wedge$ $d y \wedge d z \wedge d p \wedge d q) \neq 0$ and so the standard coordinates $(x, y, z, p, q)$ on $J^{1}\left(\mathbb{R}^{2}, \mathbb{R}\right)$ along with two additional coordinates $u, v$ may be taken as coordinates on $\Sigma_{7}$. Thus, without loss of generality, we may assume the parametrization $i_{F}$ has the form $i_{F}(x, y, z, p, q, u, v)=(x, y, z, p, q, r, s, t)$, expressing $r, s, t$ as functions of $(x, y, z, p, q, u, v)$.

The contact system $\mathcal{C}^{(2)}$ on $J^{2}\left(\mathbb{R}^{2}, \mathbb{R}\right)$ is generated by the standard contact forms

$$
\theta^{1}=d z-p d x-q d y, \quad \theta^{2}=d p-r d x-s d y, \quad \theta^{3}=d q-s d x-t d y
$$

and pulling back by $i_{F}$, we obtain a Pfaffian system (i.e. generated by 1 -forms) $I_{F}$ on $\Sigma_{7}$,

$$
I_{F}=i_{F}^{*}\left(\mathcal{C}^{(2)}\right)=\left\{\omega^{1}, \omega^{2}, \omega^{3}\right\},
$$

where $\omega^{\alpha}=i_{F}^{*} \theta^{\alpha}$. There is a correspondence between local solutions of (2.1) and local integral manifolds of $I_{F}$.

Definition 2.2. The equations (2.1) and

$$
\bar{F}\left(\bar{x}, \bar{y}, \bar{z}, \frac{\partial \bar{z}}{\partial \bar{x}}, \frac{\partial \bar{z}}{\partial \bar{y}}, \frac{\partial^{2} \bar{z}}{\partial \bar{x}^{2}}, \frac{\partial^{2} \bar{z}}{\partial \bar{x} \partial \bar{y}}, \frac{\partial^{2} \bar{z}}{\partial \bar{y}^{2}}\right)=0, \quad\left(\text { with } i_{\bar{F}}: \bar{\Sigma}_{7} \rightarrow J^{2}\left(\mathbb{R}^{2}, \mathbb{R}\right)\right)
$$

are contact-equivalent if there exists a local diffeomorphism $\phi: \Sigma_{7} \rightarrow \bar{\Sigma}_{7}$ such that $\phi^{*} I_{\bar{F}}=I_{F}$. The collection of all such maps will be denoted Contact $\left(\Sigma_{7}, \bar{\Sigma}_{7}\right)$. A contact symmetry is a selfequivalence. 
Remark 2.1. More precisely, the above definition refers to internal contact-equivalence. There is another natural notion of equivalence: namely, (2.1) and (2.2) are externally contact-equivalent if there exists a local diffeomorphism $\rho: J^{2}\left(\mathbb{R}^{2}, \mathbb{R}\right) \rightarrow J^{2}\left(\mathbb{R}^{2}, \mathbb{R}\right)$ that restricts to a local diffeomorphism $\tilde{\rho}: i_{F}\left(\Sigma_{7}\right) \rightarrow i_{\bar{F}}\left(\bar{\Sigma}_{7}\right)$ and preserves the contact system, i.e. $\rho^{*}\left(\mathcal{C}^{(2)}\right)=\mathcal{C}^{(2)}$. It is clear that any external equivalence induces a corresponding internal equivalence, but in general the converse need not hold. The difference between these two natural notions of equivalence is in general quite subtle and has been investigated in detail in [1]. A corollary of their results (c.f. Theorem 18 therein) is that for (2.1), under the maximal rank and transversality conditions, any internal equivalence extends to an external equivalence, and thus the correspondence is one-to-one.

As shown by Gardner [9], the (pointwise) classification of (2.1) into mutually exclusive elliptic, parabolic and hyperbolic classes is in fact a contact-invariant classification which arises through invariants (i.e. rank and index) of a (conformal class of a) symmetric $C^{\infty}\left(\Sigma_{7}\right)$-bilinear form $\langle\cdot, \cdot\rangle_{7}$ on $I_{F}$, namely

$$
\langle\varphi, \psi\rangle_{7} \operatorname{Vol}_{\Sigma_{7}}:=d \varphi \wedge d \psi \wedge \omega^{1} \wedge \omega^{2} \wedge \omega^{3}
$$

where $\operatorname{Vol}_{\Sigma_{7}}$ denotes any volume form on $\Sigma_{7}$. Since $i_{F}^{*}$ is surjective, there exists a 7 -form $\nu$ on $J^{2}\left(\mathbb{R}^{2}, \mathbb{R}\right)$ such that $i_{F}^{*} \nu=\operatorname{Vol}_{\Sigma_{7}}$, and so

$$
\langle\varphi, \psi\rangle_{7} i_{F}^{*} \nu=i_{F}^{*}\left(d \tilde{\varphi} \wedge d \tilde{\psi} \wedge \theta^{1} \wedge \theta^{2} \wedge \theta^{3}\right),
$$

where $\tilde{\varphi}$ and $\tilde{\psi}$ are any forms on $J^{2}\left(\mathbb{R}^{2}, \mathbb{R}\right)$ such that $\varphi=i_{F}^{*} \tilde{\varphi}$ and $\psi=i_{F}^{*} \tilde{\psi}$. Since $i_{F *}: T \Sigma_{7} \rightarrow$ $T J^{2}\left(\mathbb{R}^{2}, \mathbb{R}\right)$ is rank 7 (as is $\left.i_{F}^{*}: T^{*} J^{2}\left(\mathbb{R}^{2}, \mathbb{R}\right) \rightarrow T^{*} \Sigma_{7}\right)$ and $i_{F}^{*} d F=0$, then $\operatorname{ker}\left(i_{F}^{*}\right)=\{d F\}$, and

$$
i_{F}^{*} \eta=0 \quad \text { iff } \quad \eta \wedge d F=0, \quad \forall \eta \in \Omega^{*}\left(J^{2}\left(\mathbb{R}^{2}, \mathbb{R}\right)\right) .
$$

Consequently, letting $\operatorname{Vol}_{J^{2}\left(\mathbb{R}^{2}, \mathbb{R}\right)}=\nu \wedge d F$, we see that (2.3) is equivalent to

$$
\left(\langle\varphi, \psi\rangle_{7}\right)_{p}\left(\operatorname{Vol}_{J^{2}\left(\mathbb{R}^{2}, \mathbb{R}\right)}\right)_{i_{F}(p)}=\left(d \tilde{\varphi} \wedge d \tilde{\psi} \wedge \theta^{1} \wedge \theta^{2} \wedge \theta^{3} \wedge d F\right)_{i_{F}(p)},
$$

where $p \in \Sigma_{7}$. This definition is well-defined: it is independent of the choice of $\tilde{\varphi}$ and $\tilde{\psi}$ so long as $\varphi=i_{F}^{*} \tilde{\varphi}$ and $\psi=i_{F}^{*} \tilde{\psi}$.

A computation in the basis $\omega^{1}, \omega^{2}, \omega^{3}$ reveals that a volume form may be chosen so that

$$
\left(\left\langle\omega^{\alpha}, \omega^{\beta}\right\rangle_{7}\right)_{p}=\left(\begin{array}{ccc}
0 & 0 & 0 \\
0 & F_{t} & -\frac{1}{2} F_{s} \\
0 & -\frac{1}{2} F_{s} & F_{r}
\end{array}\right)_{i_{F}(p)} .
$$

Our assumption that $i_{F}$ have maximal rank implies that $\langle\cdot, \cdot\rangle_{7}$ cannot have rank zero. Defining

$$
\Delta=i_{F}^{*}\left(F_{r} F_{t}-\frac{1}{4} F_{s}^{2}\right)
$$

we have the following mutually exclusive cases at each point $p \in \Sigma_{7}$ :

Table 1. Contact-invariant classification of scalar second order PDE in the plane.

\begin{tabular}{|c|c|c|}
\hline elliptic & parabolic & hyperbolic \\
\hline \hline$\Delta(p)>0$ & $\Delta(p)=0$ & $\Delta(p)<0$ \\
\hline
\end{tabular}

By the commutativity of pullbacks with $d$, it is clear that this classification is a priori contactinvariant. We remark that in the classical literature on the geometry of hyperbolic equations, 
the terminology Monge characteristics appears. These are determined by the roots of the characteristic equation

$$
\lambda^{2}-F_{s} \lambda+F_{t} F_{r}=0 .
$$

The discriminant of this equation (with the coefficients evaluated on $F=0$ ) is precisely $-\frac{1}{4} \Delta$, and so the elliptic, parabolic, and hyperbolic cases correspond to the existence of no roots, a double root, and two distinct roots respectively.

In the analysis to follow, all constructions for a PDE $F=0$ will implicitly be repeated for a second $\operatorname{PDE} \bar{F}=0$ (if present). We will concern ourselves exclusively with the hyperbolic case, that is, an open subset of $\Sigma_{7}$ on which $F=0$ is hyperbolic. By the hyperbolicity condition, the two nonzero eigenvalues of $\langle\cdot, \cdot\rangle_{7}$ have opposite sign, and hence there exists a pair of rank two maximally isotropic subsystems $M_{1}$ and $M_{2}$ of $I_{F}$ at every point of consideration.

Definition 2.3. Given hyperbolic PDE $F=0$ and $\bar{F}=0$, define

$$
\begin{aligned}
& \operatorname{Contact}^{+}\left(\Sigma_{7}, \bar{\Sigma}_{7}\right)=\left\{\phi \in \operatorname{Contact}\left(\Sigma_{7}, \bar{\Sigma}_{7}\right): \phi^{*} \bar{M}_{1}=M_{1}, \phi^{*} \bar{M}_{2}=M_{2}\right\}, \\
& \operatorname{Contact}^{-}\left(\Sigma_{7}, \bar{\Sigma}_{7}\right)=\left\{\phi \in \operatorname{Contact}\left(\Sigma_{7}, \bar{\Sigma}_{7}\right): \phi^{*} \bar{M}_{1}=M_{2}, \phi^{*} \bar{M}_{2}=M_{1}\right\} .
\end{aligned}
$$

If $\bar{F}=F$, we take $\bar{\Sigma}_{7}=\Sigma_{7}$ and use the notation $\operatorname{Aut}\left(I_{F}\right):=\operatorname{Contact}\left(\Sigma_{7}, \Sigma_{7}\right)$, etc.

Remark 2.2. Implicitly, given the Pfaffian system $I_{F}$ corresponding to a hyperbolic PDE $F=0$, we assume that a choice of labelling for the $M_{1}$ and $M_{2}$ characteristics has been made. This is of course not intrinsic. All of our final results will not depend on this choice.

Both Vranceanu [25] and Gardner-Kamran [11] consider only local diffeomorphisms which preserve each of $M_{1}$ and $M_{2}$.

Example 2.1. For the wave equation written as $z_{x y}=0$, we have the pullbacks of the contact forms on $J^{2}\left(\mathbb{R}^{2}, \mathbb{R}\right)$ to the parameter space $\Sigma_{7}:(x, y, z, p, q, r, t)$,

$$
\omega^{1}=d z-p d x-q d y, \quad \omega^{2}=d p-r d x, \quad \omega^{3}=d q-t d y
$$

and

$$
\left(\left\langle\omega^{\alpha}, \omega^{\beta}\right\rangle_{7}\right)_{p}=\left(\begin{array}{ccc}
0 & 0 & 0 \\
0 & 0 & -\frac{1}{2} \\
0 & -\frac{1}{2} & 0
\end{array}\right) .
$$

Thus, $M_{1}=\left\{\omega^{1}, \omega^{2}\right\}$ and $M_{2}=\left\{\omega^{1}, \omega^{3}\right\}$. Interchanging the independent variables induces $\phi_{0}: \Sigma_{7} \rightarrow \Sigma_{7},(x, y, z, p, q, r, t) \mapsto(y, x, z, q, p, t, r)$, which satisfies

$$
\phi_{0}^{*} \omega^{1}=\omega^{1}, \quad \phi_{0}^{*} \omega^{2}=\omega^{3}, \quad \phi_{0}^{*} \omega^{3}=\omega^{2},
$$

and hence $\phi_{0} \in \operatorname{Aut}^{-}\left(I_{F}\right)$.

The hyperbolicity condition implies that there exists a local basis of $I_{F}$ which by abuse of notation we also denote $\omega^{1}, \omega^{2}, \omega^{3}$ such that

$$
M_{1}=\left\{\omega^{1}, \omega^{2}\right\}, \quad M_{2}=\left\{\omega^{1}, \omega^{3}\right\}
$$

and the matrix representing $\langle\cdot, \cdot\rangle_{7}$ is in Witt normal form

$$
\left(\left\langle\omega^{\alpha}, \omega^{\beta}\right\rangle_{7}\right)_{p}=\left(\begin{array}{ccc}
0 & 0 & 0 \\
0 & 0 & 1 \\
0 & 1 & 0
\end{array}\right) .
$$


Lemma 2.1 (Preliminary hyperbolic structure equations). There exists a (local) coframe $\boldsymbol{\omega}=\left\{\omega^{i}\right\}_{i=1}^{7}$ on $\Sigma_{7}$ such that $I_{F}=\left\{\omega^{1}, \omega^{2}, \omega^{3}\right\}$ and

$$
\begin{aligned}
& d \omega^{1} \equiv 0, \\
& d \omega^{2} \equiv \omega^{4} \wedge \omega^{5}, \\
& d \omega^{3} \equiv \omega^{6} \wedge \omega^{7},
\end{aligned}
$$

with

$$
\omega^{1} \wedge \omega^{2} \wedge \omega^{3} \wedge \omega^{4} \wedge \omega^{5} \wedge \omega^{6} \wedge \omega^{7} \neq 0 .
$$

Proof. In Theorem 1.7 in [3], an algebraic normal form for a 2 -form $\Omega$ is given. In particular, if $\Omega \wedge \Omega=0$, then $\Omega=\sigma^{1} \wedge \sigma^{2}$ is decomposable. This statement is also true in a relative sense: if $\Omega \wedge \Omega \equiv 0 \bmod I$, then $\Omega \equiv \sigma^{1} \wedge \sigma^{2} \bmod I$, where $I$ is an ideal in the exterior algebra.

Using this fact, let us deduce consequences of the Witt normal form. By definition of $\langle\cdot, \cdot\rangle_{7}$, we have (taking congruences below modulo $I_{F}$ )

$$
\begin{array}{ll}
\left\langle\omega^{2}, \omega^{2}\right\rangle_{7}=0 & \Leftrightarrow \quad d \omega^{2} \wedge d \omega^{2} \equiv 0 \quad \Leftrightarrow \quad d \omega^{2} \equiv \omega^{4} \wedge \omega^{5} \\
\left\langle\omega^{3}, \omega^{3}\right\rangle_{7}=0 & \Leftrightarrow \quad d \omega^{3} \wedge d \omega^{3} \equiv 0 \quad \Leftrightarrow \quad d \omega^{3} \equiv \omega^{6} \wedge \omega^{7}, \\
\left\langle\omega^{2}, \omega^{3}\right\rangle_{7}=1 & \Leftrightarrow \quad d \omega^{2} \wedge d \omega^{3} \wedge \omega^{1} \wedge \omega^{2} \wedge \omega^{3}=\omega^{4} \wedge \omega^{5} \wedge \omega^{6} \wedge \omega^{7} \wedge \omega^{1} \wedge \omega^{2} \wedge \omega^{3} \neq 0 .
\end{array}
$$

Using $\left\langle\omega^{1}, \omega^{2}\right\rangle_{7}=\left\langle\omega^{1}, \omega^{3}\right\rangle_{7}=0$, we have

$$
\begin{aligned}
& 0=d \omega^{1} \wedge d \omega^{2} \wedge \omega^{1} \wedge \omega^{2} \wedge \omega^{3}=d \omega^{1} \wedge \omega^{4} \wedge \omega^{5} \wedge \omega^{1} \wedge \omega^{2} \wedge \omega^{3}, \\
& 0=d \omega^{1} \wedge d \omega^{3} \wedge \omega^{1} \wedge \omega^{2} \wedge \omega^{3}=d \omega^{1} \wedge \omega^{6} \wedge \omega^{7} \wedge \omega^{1} \wedge \omega^{2} \wedge \omega^{3},
\end{aligned}
$$

and thus $d \omega^{1} \equiv 0$.

Consequently, $\left\{\omega^{i}\right\}_{i=1}^{7}$ is a (local) coframe on $\Sigma_{7}$, and the structure equations can be written

$$
d \omega^{i}=\frac{1}{2} \gamma^{i}{ }_{j k} \omega^{j} \wedge \omega^{k}
$$

\section{Monge-Ampère, Goursat and generic hyperbolic equations}

The congruences appearing in the preliminary hyperbolic structure equations can be tightened with a more careful study of integrability conditions and further adaptations of the coframe. The details are provided in Appendix A.

Theorem 3.1 (Hyperbolic structure equations). Given any hyperbolic equation $F=0$ with nondegenerate parametrization $i_{F}: \Sigma_{7} \rightarrow J^{2}\left(\mathbb{R}^{2}, \mathbb{R}\right)$, there is an associated coframe $\boldsymbol{\omega}=\left\{\omega^{i}\right\}_{i=1}^{7}$ on $\Sigma_{7}$ such that

1. $I_{F}=\left\{\omega^{1}, \omega^{2}, \omega^{3}\right\}, M_{1}=\left\{\omega^{1}, \omega^{2}\right\}, M_{2}=\left\{\omega^{1}, \omega^{3}\right\}$.

2. We have the structure equations

$$
\begin{aligned}
& d \omega^{1} \equiv \omega^{3} \wedge \omega^{6}+\omega^{2} \wedge \omega^{4} \quad \bmod \left\{\omega^{1}\right\}, \\
& d \omega^{2} \equiv \omega^{4} \wedge \omega^{5}+U_{1} \omega^{3} \wedge \omega^{7} \quad \bmod \left\{\omega^{1}, \omega^{2}\right\}, \\
& d \omega^{3} \equiv \omega^{6} \wedge \omega^{7}+U_{2} \omega^{2} \wedge \omega^{5} \quad \bmod \left\{\omega^{1}, \omega^{3}\right\}
\end{aligned}
$$

for some functions $U_{1}, U_{2}$ on $\Sigma_{7}$. 
A finer contact-invariant classification of hyperbolic equations arises from the study of the class of $M_{1}$ and $M_{2}$. Let us recall some basic definitions.

Definition 3.1. Let $I$ be a Pfaffian system on a manifold $\Sigma$. Define the

1. Cauchy characteristic space $\left.\mathcal{A}(I)=\left\{X \in \mathfrak{X}(\Sigma): X \in I^{\perp}, X\right\lrcorner d I \subset I\right\}$.

2. Cartan system $\mathcal{C}(I)=\mathcal{A}(I)^{\perp}$. The class of $I$ is the rank of $\mathcal{C}(I)$ (as a $C^{\infty}(\Sigma)$-module).

Here, $\perp$ refers to the annihilator submodule.

The hyperbolic structure equations indicate that there are only two possibilities for the class of $M_{1}$ and $M_{2}$.

Corollary 3.1. For $i=1,2$, $\operatorname{class}\left(M_{i}\right)=6$ or 7 . Moreover, $\operatorname{class}\left(M_{i}\right)=6$ iff $U_{i}=0$.

Proof. Let $\left\{\frac{\partial}{\partial \omega^{i}}\right\}_{i=1}^{7}$ denote the dual basis to $\left\{\omega^{i}\right\}_{i=1}^{7}$. From (3.1), we have

$$
\mathcal{A}\left(M_{1}\right) \subset\left\{\frac{\partial}{\partial \omega^{7}}\right\}, \quad \mathcal{A}\left(M_{2}\right) \subset\left\{\frac{\partial}{\partial \omega^{5}}\right\} .
$$

Moreover, $\operatorname{class}\left(M_{1}\right)=6$ iff $\frac{\partial}{\partial \omega^{7}} \in \mathcal{A}\left(M_{1}\right)$ iff $U_{1}=0$. Similarly for $M_{2}$.

Consequently, we obtain the subclassification of hyperbolic equations given in Table 2.

Table 2. Contact-invariant classification of hyperbolic PDE.

\begin{tabular}{|c|c|}
\hline Type & Contact-invariant classification \\
\hline \hline Monge-Ampère (6-6) & $\operatorname{class}\left(M_{1}\right)=\operatorname{class}\left(M_{2}\right)=6$ \\
Goursat (6-7) & $\left\{\operatorname{class}\left(M_{1}\right), \operatorname{class}\left(M_{2}\right)\right\}=\{6,7\}$ \\
generic $(7-7)$ & $\operatorname{class}\left(M_{1}\right)=\operatorname{class}\left(M_{2}\right)=7$ \\
\hline
\end{tabular}

Example 3.1. We give some known examples of each type of hyperbolic equation:

- Monge-Ampère: wave equation $z_{x y}=0$, Liouville equation $z_{x y}=e^{z}$, Klein-Gordon equation $z_{x y}=z$, or more generally the $f$-Gordon equation $z_{x y}=f\left(x, y, z, z_{x}, z_{y}\right)$, and MongeAmpère equation $z_{x x} z_{y y}-\left(z_{x y}\right)^{2}=f(x, y)$.

- Goursat: $z_{x x}=f\left(z_{x y}\right)$ where $f^{\prime \prime} \neq 0$.

- generic: $z_{x y}=\frac{1}{2} \sin \left(z_{x x}\right) \cos \left(z_{y y}\right)$, or $3 z_{x x}\left(z_{y y}\right)^{3}+1=0$.

The terminology for class 6-6 equations is justified by the following result, known to Vranceanu [26]. We refer the reader to Gardner-Kamran [11] for a modern proof.

Theorem 3.2. A second-order hyperbolic equation has $\operatorname{class}\left(M_{i}\right)=6, i=1,2$ if and only if its locus can be given by an equation of the form

$$
a\left(z_{x x} z_{y y}-\left(z_{x y}\right)^{2}\right)+b z_{x x}+2 c z_{x y}+d z_{y y}+e=0,
$$

where $a, b, c, d$, e are functions of $x, y, z, z_{x}, z_{y}$.

The examples given above were obtained by constructing explicit coframes which realize the abstract structure equations given in Theorem 3.1, which in general is a very tedious task and is equation-specific. We state here two relative invariants $I_{1}, I_{2}$ (which are related to the two 
relative invariants $U_{1}, U_{2}$ ) whose vanishing/nonvanishing determine the type of any hyperbolic equation. Given any hyperbolic equation $F=0$, define

$$
\lambda_{ \pm}=\frac{F_{s}}{2} \pm \sqrt{|\Delta|}
$$

which are the roots of the characteristic equation (2.6). Without loss of generality, we may assume that $F_{s} \geq 0$. (If not, take $\hat{F}=-F$ instead.) By the hyperbolicity assumption $\lambda_{+}>0$. The proof of the following theorem is given in Appendix B.

Theorem 3.3 (Relative contact invariants for hyperbolic equations). Suppose that $F=$ 0 is a hyperbolic equation with $F_{s} \geq 0$. Let

$$
\tilde{I}_{1}=\operatorname{det}\left(\begin{array}{ccc}
F_{r} & F_{s} & F_{t} \\
\lambda_{+} & F_{t} & 0 \\
\left(\frac{F_{t}}{\lambda_{+}}\right)_{r} & \left(\frac{F_{t}}{\lambda_{+}}\right)_{s} & \left(\frac{F_{t}}{\lambda_{+}}\right)_{t}
\end{array}\right), \quad \tilde{I}_{2}=\operatorname{det}\left(\begin{array}{ccc}
0 & F_{r} & \lambda_{+} \\
F_{r} & F_{s} & F_{t} \\
\left(\frac{F_{r}}{\lambda_{+}}\right)_{r} & \left(\frac{F_{r}}{\lambda_{+}}\right)_{s} & \left(\frac{F_{r}}{\lambda_{+}}\right)_{t}
\end{array}\right),
$$

and $I_{i}=i_{F}^{*} \tilde{I}_{i}$. Then we have the following classification of $F=0$ :

\begin{tabular}{|c|c|}
\hline Type & Contact-invariant classification \\
\hline \hline Monge-Ampère & $I_{1}=I_{2}=0$ \\
Goursat & exactly one of $I_{1}$ or $I_{2}$ is zero \\
generic & $I_{1} I_{2} \neq 0$ \\
\hline
\end{tabular}

Moreover, we have the scaling property: If $\phi$ is a function on $J^{2}\left(\mathbb{R}^{2}, \mathbb{R}\right)$ such that $i_{F}^{*} \phi>0$, then

$$
\hat{F}=\phi F \quad \Rightarrow \quad \hat{I}_{i}=\left(i_{F}^{*} \phi\right)^{2} I_{i}, \quad i=1,2 .
$$

We note that the scaling property is a fundamental property of these relative invariants: their vanishing/nonvanishing depends only on the equation locus.

Remark 3.1. For a general hyperbolic equation $F(x, y, z, p, q, r, s, t)=0$, Juráš' [13] calculated two (relative) invariants $M_{\sigma}, M_{\tau}$ whose vanishing characterizes the Monge-Ampère class. His invariants were given explicitly in terms of two non-proportional real roots $(\mu, \lambda)=\left(m_{x}, m_{y}\right)$ and $(\mu, \lambda)=\left(n_{x}, n_{y}\right)$ of the characteristic equation

$$
F_{r} \lambda^{2}-F_{s} \lambda \mu+F_{t} \mu^{2}=0,
$$

which he associates to the given PDE. We note here that the characteristic equation (2.6) that we have used differs from that of Juráš (but has the same discriminant). Our invariants $I_{1}, I_{2}$ appear to be simpler written in this determinantal form.

Using the relative contact invariants $I_{1}, I_{2}$ we can identify some more general examples of hyperbolic equations of Goursat and generic type.

Corollary 3.2. The classification of hyperbolic equations of the form $F(x, y, z, p, q, r, t)=0$, $G(x, y, z, p, q, r, s)=0$, and $r t=f(x, y, z, p, q, s)$ is given in Table 3 below.

Proof. The hyperbolicity condition in each case is clear. Define the function

$$
\Delta_{r, t}^{F}=F_{r}^{2} F_{t t}-2 F_{r} F_{t} F_{r t}+F_{t}^{2} F_{r r}
$$

and similarly for $\Delta_{r, s}^{G}$. Without loss of generality $G_{s}, f_{s} \geq 0$. The calculation of $\tilde{I}_{1}, \tilde{I}_{2}$ leads to

$$
F(x, y, z, p, q, r, t)=0: \quad \tilde{I}_{1}=\frac{-F_{t}^{2} \Delta_{r, t}^{F}}{2\left(-F_{t} F_{r}\right)^{3 / 2}}, \quad \tilde{I}_{2}=\frac{-F_{r}{ }^{2} \Delta_{r, t}^{F}}{2\left(-F_{t} F_{r}\right)^{3 / 2}},
$$




$$
\begin{array}{lll}
G(x, y, z, p, q, r, s)=0: & \tilde{I}_{1}=0, & \tilde{I}_{2}=\frac{-\Delta_{r, s}^{G}}{G_{s}} \\
r t=f(x, y, z, p, q, s): & \tilde{I}_{1}=\frac{\left(f_{s s}-2\right) r^{2}}{\sqrt{f_{s}^{2}-4 r t}}, & \tilde{I}_{2}=\frac{\left(f_{s s}-2\right) t^{2}}{\sqrt{f_{s}^{2}-4 r t}}, \\
r t=-f(x, y, z, p, q, s): & \tilde{I}_{1}=\frac{-\left(f_{s s}+2\right) r^{2}}{\sqrt{f_{s}^{2}-4 r t}}, & \tilde{I}_{2}=\frac{-\left(f_{s s}+2\right) t^{2}}{\sqrt{f_{s}^{2}-4 r t}} .
\end{array}
$$

For $F(x, y, z, p, q, r, t)=0$ : Since $i_{F}^{*}\left(F_{t} F_{r}\right)<0$, then either $I_{1}, I_{2}$ both vanish or both do not vanish, i.e. either class 6-6 or class 7-7. The vanishing of $I_{1}, I_{2}$ is completely characterized by the vanishing of $i_{F}^{*}\left(\Delta_{r, t}^{F}\right)$. By Theorem 3.2, we know what all class 6-6 equations of the form $F(x, y, z, p, q, r, t)=0$ look like. Hence,

$$
i_{F}^{*}\left(\Delta_{r, t}^{F}\right)=0 \quad \text { iff its locus can be given by } \quad F(x, y, z, p, q, r, t)=a r+b t+c=0,
$$

where $a, b, c$ are functions of $x, y, z, p, q$ only. The proof for $G(x, y, z, p, q, r, s)=0$ is similar and the result for the last equation is immediate.

Table 3. General examples of hyperbolic equations and their types.

\begin{tabular}{|c|c|c|}
\hline Equation & $\begin{array}{c}\text { Hyperbolicity } \\
\text { condition }\end{array}$ & Type \\
\hline \hline$F(x, y, z, p, q, r, t)=0$ & $i_{F}^{*}\left(F_{r} F_{t}\right)<0$ & $\begin{array}{c}\text { 6-6 iff } F \text { is an affine function of } r, t\left(^{*}\right) \\
7-7 \text { otherwise }\end{array}$ \\
\hline$G(x, y, z, p, q, r, s)=0$ & $i_{G}^{*}\left(G_{s}\right) \neq 0$ & $\begin{array}{c}6-6 \text { iff } G \text { is an affine function of } r, s\left(^{*}\right) \\
6-7 \text { otherwise }\end{array}$ \\
\hline$r t=f(x, y, z, p, q, s)$ & $4 f<f_{s}{ }^{2}$ & $\begin{array}{c}\text { Assuming } r t \neq 0: \\
6-6 \text { iff } f_{s s}=2 \\
\end{array}$ \\
& & $7-7$ iff $f_{s s} \neq 2$ \\
\hline
\end{tabular}

$(*)$ More precisely, it is the zero-locus of such a function.

Remark 3.2. Hyperbolic equations of Goursat and generic type are necessarily non-variational. This is because a variational formulation for a second order PDE requires a first order Lagrangian (density) $L(x, y, z, p, q)$ and the corresponding Euler-Lagrange equation is

$$
\frac{\partial L}{\partial z}-D_{x}\left(\frac{\partial L}{\partial p}\right)-D_{y}\left(\frac{\partial L}{\partial q}\right)=0
$$

where $D_{x}$ and $D_{y}$ are total derivative operators

$$
D_{x}=\frac{\partial}{\partial x}+p \frac{\partial}{\partial z}+r \frac{\partial}{\partial p}+s \frac{\partial}{\partial q}, \quad D_{y}=\frac{\partial}{\partial y}+q \frac{\partial}{\partial z}+s \frac{\partial}{\partial p}+t \frac{\partial}{\partial q} .
$$

Thus, the Euler-Lagrange equation is quasi-linear and, if hyperbolic, is of Monge-Ampère type.

For the remainder of the paper we will deal exclusively with the generic case. In this case $U_{1}, U_{2}$ in (3.1) are nonzero and can be further normalized through a coframe adaptation. Before carrying out this normalization, we recall some more basic definitions.

Definition 3.2. Given a Pfaffian system $I$ on a manifold $\Sigma$, recall that the first derived system $I^{(1)} \subset I$ is the Pfaffian system defined by the short exact sequence

$$
0 \longrightarrow I^{(1)} \longleftrightarrow I \stackrel{\pi \circ d}{\longrightarrow} d I \bmod I \longrightarrow 0,
$$


where $\pi: \Omega^{*}(\Sigma) \rightarrow \Omega^{*}(\Sigma) / I$ be the canonical surjection. (Here we abuse notation and identify $I$ with the algebraic ideal in $\Omega^{*}(\Sigma)$ that it generates.) Iteratively, we define the derived flag $\cdots \subset I^{(k)} \subset \cdots \subset I^{(1)} \subset I$.

Remark 3.3. $I$ is completely integrable (in the Frobenius sense) iff $I^{(1)}=I$.

Since $d$ commutes with pullbacks, each derived system $I^{(k)}$ is invariant under any automorphism of $I$, i.e. if $\phi \in \operatorname{Aut}(I)$, then $\phi^{*} I^{(k)}=I^{(k)}$.

Definition 3.3. For hyperbolic equations, define

$$
\begin{aligned}
& \left.\operatorname{Char}\left(I_{F}, d M_{i}\right)=\left\{X \in \mathfrak{X}\left(\Sigma_{7}\right): X \in I_{F}^{\perp}, X\right\lrcorner d M_{i} \subset I_{F}\right\}, \\
& C\left(I_{F}, d M_{i}\right)=\operatorname{Char}\left(I_{F}, d M_{i}\right)^{\perp} .
\end{aligned}
$$

We now normalize the coefficients $U_{1}, U_{2}$ in the generic case. Moreover, explicit generators for the first few systems in the derived flag of $C\left(I_{F}, d M_{1}\right)$ and $C\left(I_{F}, d M_{2}\right)$ are obtained. The proofs of the following theorem and subsequent corollaries are provided in Appendix $\mathrm{C}$.

Theorem 3.4 (Generic hyperbolic structure equations). Given any generic hyperbolic equation $F=0$ with nondegenerate parametrization $i_{F}: \Sigma_{7} \rightarrow J^{2}\left(\mathbb{R}^{2}, \mathbb{R}\right)$, there is an associated coframe $\boldsymbol{\omega}=\left\{\omega^{i}\right\}_{i=1}^{7}$ on $\Sigma_{7}$ such that

1) $I_{F}=\left\{\omega^{1}, \omega^{2}, \omega^{3}\right\}, I_{F}^{(1)}=\left\{\omega^{1}\right\}, M_{1}=\left\{\omega^{1}, \omega^{2}\right\}, M_{2}=\left\{\omega^{1}, \omega^{3}\right\}$,

2) we have the structure equations

$$
\begin{array}{rlrl}
d \omega^{1} & \equiv \omega^{3} \wedge \omega^{6}+\omega^{2} \wedge \omega^{4} & & \bmod I_{F}^{(1)}, \\
d \omega^{2} \equiv \omega^{4} \wedge \omega^{5}+\omega^{3} \wedge \omega^{7} & & \bmod M_{1} \\
d \omega^{3} & \equiv \omega^{6} \wedge \omega^{7}+\epsilon \omega^{2} \wedge \omega^{5} & & \bmod M_{2},
\end{array}
$$

where $\epsilon= \pm 1$,

3) for some choice of coframe satisfying the above structure equations, we have

$$
\begin{array}{ll}
C\left(I_{F}, d M_{1}\right)=\left\{\omega^{1}, \omega^{2}, \omega^{3}, \omega^{4}, \omega^{5}\right\}, & C\left(I_{F}, d M_{2}\right)=\left\{\omega^{1}, \omega^{2}, \omega^{3}, \omega^{6}, \omega^{7}\right\}, \\
C\left(I_{F}, d M_{1}\right)^{(1)}=\left\{\omega^{1}, \omega^{2}, \omega^{4}, \omega^{5}\right\}, & C\left(I_{F}, d M_{2}\right)^{(1)}=\left\{\omega^{1}, \omega^{3}, \omega^{6}, \omega^{7}\right\}, \\
C\left(I_{F}, d M_{1}\right)^{(2)}=\left\{\omega^{4}, \omega^{5}\right\}, & C\left(I_{F}, d M_{2}\right)^{(2)}=\left\{\omega^{6}, \omega^{7}\right\} .
\end{array}
$$

Corollary 3.3. For the choice of coframe as in Theorem 3.4, we have the additional structure equations

$$
\begin{array}{ll}
d \omega^{4} \equiv \epsilon \omega^{5} \wedge \omega^{6} & \bmod \left\{\omega^{1}, \omega^{2}, \omega^{4}\right\}, \\
d \omega^{5} \equiv 0 & \bmod \left\{\omega^{1}, \omega^{2}, \omega^{4}, \omega^{5}\right\}, \\
d \omega^{6} \equiv-\omega^{4} \wedge \omega^{7} & \bmod \left\{\omega^{1}, \omega^{3}, \omega^{6}\right\}, \\
d \omega^{7} \equiv 0 & \bmod \left\{\omega^{1}, \omega^{3}, \omega^{6}, \omega^{7}\right\} .
\end{array}
$$

We will refer to (3.2) and (3.4) collectively as the generic hyperbolic structure equations.

Corollary 3.4. $\epsilon$ is a contact invariant, and moreover $\epsilon=\operatorname{sgn}\left(I_{1} I_{2}\right)$.

Example 3.2. From Table 3 and the proof of Corollary 3.2, we see $\epsilon=1$ for:

- $F(x, y, z, p, q, r, t)=0$ whenever $F$ is not an affine function of $r, t$.

- $r t=f(x, y, z, p, q, s)$ whenever $f_{s s} \neq 2$. 
Remark 3.4. We have the following dictionary of notations for the adapted coframe labelling:

\begin{tabular}{c|c|c|c} 
& Gardner-Kamran [11] & Vranceanu [25] & The \\
\hline$I_{F}$ & $\omega^{1}, \pi^{2}, \pi^{3}$ & $d s^{1}, d s^{2}, d s^{3}$ & $\omega^{1}, \omega^{2}, \omega^{3}$ \\
$M_{1}$ & $\omega^{1}, \pi^{2}$ & $d s^{1}, d s^{2}$ & $\omega^{1}, \omega^{2}$ \\
$M_{2}$ & $\omega^{1}, \pi^{3}$ & $d s^{1}, d s^{3}$ & $\omega^{1}, \omega^{3}$ \\
$C\left(I_{F}, d M_{1}\right)$ & $\omega^{1}, \pi^{2}, \pi^{3}, \omega^{4}, \omega^{5}$ & $d s^{1}, d s^{2}, d s^{3}, d s^{5}, d s^{6}$ & $\omega^{1}, \omega^{2}, \omega^{3}, \omega^{4}, \omega^{5}$ \\
$C\left(I_{F}, d M_{2}\right)$ & $\omega^{1}, \pi^{2}, \pi^{3}, \omega^{6}, \omega^{7}$ & $d s^{1}, d s^{2}, d s^{3}, d s^{4}, d s^{7}$ & $\omega^{1}, \omega^{2}, \omega^{3}, \omega^{6}, \omega^{7}$
\end{tabular}

\section{The structure group and the Cartan equivalence problem}

In this section, we reformulate the problem of contact-equivalence of PDE as a Cartan equivalence problem. The reader will notice the similarities in the calculation of the structure group in this section and in the calculations in the proof of Corollary 3.4 provided in Appendix C.

For any $\phi \in \operatorname{Contact}^{+}\left(\Sigma_{7}, \bar{\Sigma}_{7}\right)$,

$$
\phi^{*} I_{\bar{F}}^{(1)}=I_{F}^{(1)}, \quad \phi^{*}\left(C\left(I_{\bar{F}}, d \bar{M}_{i}\right)^{(k)}\right)=C\left(I_{F}, d M_{i}\right)^{(k)}, \quad i=1,2, \quad \forall k \geq 0 .
$$

Consequently, with respect to the adapted coframe $\boldsymbol{\omega}$ on $\Sigma_{7}$ (as specified in Theorem 3.4) and corresponding coframe $\overline{\boldsymbol{\omega}}$ on $\bar{\Sigma}_{7}$, we have

$$
\phi^{*}\left(\begin{array}{c}
\bar{\omega}^{1} \\
\bar{\omega}^{2} \\
\bar{\omega}^{3} \\
\bar{\omega}^{4} \\
\bar{\omega}^{5} \\
\bar{\omega}^{6} \\
\bar{\omega}^{7}
\end{array}\right)=\left(\begin{array}{ccccccc}
\lambda_{1} & 0 & 0 & 0 & 0 & 0 & 0 \\
\mu_{1} & \lambda_{2} & 0 & 0 & 0 & 0 & 0 \\
\mu_{2} & 0 & \lambda_{3} & 0 & 0 & 0 & 0 \\
0 & 0 & 0 & \lambda_{4} & \nu_{1} & 0 & 0 \\
0 & 0 & 0 & \mu_{3} & \lambda_{5} & 0 & 0 \\
0 & 0 & 0 & 0 & 0 & \lambda_{6} & \nu_{2} \\
0 & 0 & 0 & 0 & 0 & \mu_{4} & \lambda_{7}
\end{array}\right)\left(\begin{array}{c}
\omega^{1} \\
\omega^{2} \\
\omega^{3} \\
\omega^{4} \\
\omega^{5} \\
\omega^{6} \\
\omega^{7}
\end{array}\right) .
$$

Applying $\phi^{*}$ to the $d \bar{\omega}^{1}$ structure equation in (3.2) yields

$$
\phi^{*} d \bar{\omega}^{1}=d \phi^{*} \bar{\omega}^{1}=d\left(\lambda_{1} \omega^{1}\right) \equiv \lambda_{1}\left(\omega^{3} \wedge \omega^{6}+\omega^{2} \wedge \omega^{4}\right) \quad \bmod I_{F}^{(1)},
$$

and also

$$
\phi^{*} d \bar{\omega}^{1} \equiv \lambda_{3} \omega^{3} \wedge\left(\lambda_{6} \omega^{6}+\nu_{2} \omega^{7}\right)+\lambda_{2} \omega^{2} \wedge\left(\lambda_{4} \omega^{4}+\nu_{1} \omega^{5}\right) \quad \bmod I_{F}^{(1)},
$$

which implies $\nu_{1}=\nu_{2}=0, \lambda_{1}=\lambda_{3} \lambda_{6}=\lambda_{2} \lambda_{4}$. Similarly, using the $d \bar{\omega}^{2}, d \bar{\omega}^{3}$ equations yields

$$
\begin{array}{ll}
\lambda_{1}=\lambda_{3} \lambda_{6}=\lambda_{2} \lambda_{4}, & \nu_{1}=\nu_{2}=0, \\
\lambda_{2}=\lambda_{4} \lambda_{5}=\lambda_{3} \lambda_{7}, & \mu_{1}=\lambda_{3} \mu_{4}, \\
\lambda_{3}=\lambda_{6} \lambda_{7}=\lambda_{2} \lambda_{5}, & \mu_{2}=\epsilon \lambda_{2} \mu_{3} .
\end{array}
$$

Then

$$
\beta:=\frac{\lambda_{6}}{\lambda_{4}}=\frac{\lambda_{2}}{\lambda_{3}}=\frac{1}{\lambda_{5}}=\frac{\lambda_{4}}{\lambda_{2}}, \quad \beta=\frac{\lambda_{2}}{\lambda_{3}}=\lambda_{7}=\frac{\lambda_{3}}{\lambda_{6}} \quad \Rightarrow \quad \beta^{4}=1 \quad \Rightarrow \quad \beta= \pm 1,
$$

and so

$$
\begin{aligned}
& \left(\lambda_{1}, \lambda_{2}, \lambda_{3}, \lambda_{4}, \lambda_{5}, \lambda_{6}, \lambda_{7}\right)=\left(\beta a_{1}^{2}, a_{1}, \beta a_{1}, \beta a_{1}, \beta, a_{1}, \beta\right), \\
& \left(\mu_{1}, \mu_{2}, \mu_{3}, \mu_{4}\right)=\left(\beta a_{1} a_{2}, \epsilon a_{1} a_{3}, a_{3}, a_{2}\right), \quad \forall a_{1} \neq 0, a_{2}, a_{3} \in \mathbb{R} .
\end{aligned}
$$


This leads us to define

$$
S=\operatorname{diag}(-1,1,-1,-1,-1,1,-1),
$$

and the connected matrix Lie group

$$
G^{0}=\left\{M(\mathbf{a}): \mathbf{a} \in \mathbb{R}^{+} \times \mathbb{R}^{2}\right\}, \quad M(\mathbf{a})=\left(\begin{array}{cccccccc}
a_{1}{ }^{2} & 0 & 0 & 0 & 0 & 0 & 0 \\
a_{1} a_{2} & a_{1} & 0 & 0 & 0 & 0 & 0 \\
\epsilon a_{1} a_{3} & 0 & a_{1} & 0 & 0 & 0 & 0 \\
0 & 0 & 0 & a_{1} & 0 & 0 & 0 \\
0 & 0 & 0 & a_{3} & 1 & 0 & 0 \\
0 & 0 & 0 & 0 & 0 & a_{1} & 0 \\
0 & 0 & 0 & 0 & 0 & a_{2} & 1
\end{array}\right) .
$$

Let us also define

$$
R=\left(\begin{array}{ccccccc}
-\epsilon & 0 & 0 & 0 & 0 & 0 & 0 \\
0 & 0 & \epsilon & 0 & 0 & 0 & 0 \\
0 & -\epsilon & 0 & 0 & 0 & 0 & 0 \\
0 & 0 & 0 & 0 & 0 & -1 & 0 \\
0 & 0 & 0 & 0 & 0 & 0 & -\epsilon \\
0 & 0 & 0 & 1 & 0 & 0 & 0 \\
0 & 0 & 0 & 0 & -\epsilon & 0 & 0
\end{array}\right) \Rightarrow R^{2}=\operatorname{diag}(1,-1,-1,-1,1,-1,1)
$$

We note that $R^{4}=S^{2}=1$ and we have the relation $R S=S R^{-1}$, and consequently $R, S$ generate the dihedral group of order 8

$$
D_{8}=\left\langle R, S: R^{4}=S^{2}=S R S R=1\right\rangle .
$$

The results (4.1) of the previous calculations establish that

$$
\phi \in \operatorname{Contact}^{+}\left(\Sigma_{7}, \bar{\Sigma}_{7}\right) \quad \text { iff } \quad \phi^{*} \overline{\boldsymbol{\omega}}=g \boldsymbol{\omega}, \quad \text { where } \quad g: \Sigma_{7} \rightarrow G^{+},
$$

where $G^{+}$is the group generated by $G^{0}, S, R^{2}$, which we can realize as the semi-direct product

$$
G^{+}=G^{0} \rtimes\left\langle S, R^{2}\right\rangle
$$

induced by the adjoint action. If $\phi_{0}^{*} \overline{\boldsymbol{\omega}}=R \boldsymbol{\omega}$, then $\phi_{0} \in \operatorname{Contact}^{-}\left(\Sigma_{7}, \bar{\Sigma}_{7}\right)$. Conversely, given any $\phi \in \operatorname{Contact}^{-}\left(\Sigma_{7}, \bar{\Sigma}_{7}\right)$, we have $\phi=\phi_{0} \circ \tilde{\phi}$, where $\tilde{\phi} \in \operatorname{Aut}^{+}\left(I_{F}\right)$. Thus,

$$
\phi^{*} \overline{\boldsymbol{\omega}}=\tilde{\phi}^{*} \phi_{0}^{*} \overline{\boldsymbol{\omega}}=\tilde{\phi}^{*} R \boldsymbol{\omega}=R g \boldsymbol{\omega}=\operatorname{Ad}_{R}(g) R \boldsymbol{\omega}, \quad \forall g \in G^{+} .
$$

Since $G^{0}$ is $\operatorname{Ad}_{R^{-}}$invariant, and $\operatorname{Ad}_{R}(S)=S R^{2}$, then $G^{+}$is $\operatorname{Ad}_{R}$-invariant and so

$$
\phi \in \operatorname{Contact}^{-}\left(\Sigma_{7}, \bar{\Sigma}_{7}\right) \quad \text { iff } \quad \phi^{*} \overline{\boldsymbol{\omega}}=g \boldsymbol{\omega}, \quad \text { where } \quad g: \Sigma_{7} \rightarrow G^{-} \text {, }
$$

where $G^{-}=G^{+} \cdot R$. (Note that $G^{-}$is not a group.) Consequently, we define

$$
G=G^{0} \rtimes D_{8},
$$

and we have established:

Lemma 4.1. $\phi \in \operatorname{Contact}\left(\Sigma_{7}, \bar{\Sigma}_{7}\right)$ if and only if

$$
\phi^{*} \overline{\boldsymbol{\omega}}=g \boldsymbol{\omega}, \quad \text { for some } \quad g: \Sigma_{7} \rightarrow G \text {. }
$$


The group $G$ will play the role of our initial structure group in the application of the Cartan equivalence method $[6,10,22]$. Specifically, the Cartan equivalence problem for generic hyperbolic equations can be stated as follows: Given the coframes $\boldsymbol{\omega}, \overline{\boldsymbol{\omega}}$ on $\Sigma_{7}, \bar{\Sigma}_{7}$ respectively, find necessary and sufficient conditions for the existence of a local diffeomorphism $\phi: \Sigma_{7} \rightarrow \bar{\Sigma}_{7}$ satisfying (4.3). This is also known as the isomorphism problem for $G$-structures $(\boldsymbol{\omega}, G)$ and $(\bar{\omega}, G)$.

Remark 4.1. Vranceanu (c.f. page 366 in [25]) considered the equivalence problem with respect to a smaller group which, in our notation, is $G^{0} \rtimes\left\langle R^{2}\right\rangle$. This has index 4 in $G$.

The solution of the general Cartan equivalence problem leads to either the structure equations of an $\{e\}$-structure or of an infinite Lie pseudo-group. However, for the equivalence problem for generic hyperbolic equations only the former case occurs. In particular, we will show in the next section that we are led to $\{e\}$-structures on $\Sigma_{7} \times G_{\Gamma}$, where $G_{\Gamma} \subset G$ is a subgroup of dimension at most two. (Different $\{e\}$-structures arise due to normalizations of nonconstant type, and will depend on choices of normal forms $\Gamma$ in different orbits.) For the moment, let us recall the general solution to the coframe ( $\{e\}$-structure) equivalence problem. Our description below is abbreviated from the presentation given in [22].

Let $\boldsymbol{\Theta}, \overline{\boldsymbol{\Theta}}$ be local coframes on manifolds $M, \bar{M}$ respectively of dimension $m$, and let $\Phi$ satisfy $\Phi^{*} \overline{\boldsymbol{\Theta}}=\boldsymbol{\Theta}$. If the structure equations for the $\{e\}$-structures are correspondingly

$$
d \Theta^{a}=\frac{1}{2} T_{b c}^{a} \Theta^{b} \wedge \Theta^{c}, \quad d \bar{\Theta}^{a}=\frac{1}{2} \bar{T}_{b c}^{a} \bar{\Theta}^{b} \wedge \bar{\Theta}^{c}, \quad 1 \leq a, b, c \leq m,
$$

then by commutativity of $\Phi^{*}$ and $d$, the structure functions $T_{b c}^{a}$ are invariants, i.e.

$$
\bar{T}_{b c}^{a} \circ \Phi=T_{b c}^{a} .
$$

For any local function $f$ on $M$, define the coframe derivatives $\frac{\partial f}{\partial \Theta^{a}}$ by

$$
d f=\frac{\partial f}{\partial \Theta^{k}} \Theta^{k}
$$

Let us write the iterated derivatives of the structure functions as

$$
T_{\sigma}=\frac{\partial^{s} T_{b c}^{a}}{\partial \Theta^{k_{s}} \cdots \partial \Theta^{k_{1}}}, \quad \text { where } \quad \sigma=\left(a, b, c, k_{1}, \ldots, k_{s}\right) \quad \text { and } \quad s=\operatorname{order}(\sigma)
$$

and $1 \leq a, b, c, k_{1}, \ldots, k_{s} \leq m$. We repeat this construction for the barred variables. Necessarily, again as a consequence of commutativity of $\Phi^{*}$ and $d$, the derived structure functions $T_{\sigma}$ and $\bar{T}_{\sigma}$ satisfy the invariance equations

$$
\bar{T}_{\sigma}(\bar{x})=T_{\sigma}(x), \quad \text { when } \quad \bar{x}=\Phi(x), \quad \forall \operatorname{order}(\sigma) \geq 0 .
$$

Note that these equations are not independent: there are generalized Jacobi identities (which we will not describe explicitly here) which allow the permutation of the coframe derivatives, so in general only nondecreasing coframe derivative indices are needed.

Definition 4.1. Let $\Theta$ be a coframe with defined on an open set $U \subset M$.

1. Let $\mathbb{K}^{(s)}$ be the Euclidean space of dimension equal to the number of multi-indices

$$
\sigma=\left(a, b, c, k_{1}, \ldots, k_{r}\right), \quad b<c, \quad k_{1} \leq \cdots \leq k_{r}, \quad 0 \leq r \leq s .
$$

2. The $s^{\text {th }}$ order structure map associated to $\Theta$ is

$$
\mathbf{T}^{(s)}: U \rightarrow \mathbb{K}^{(s)}, \quad z_{\sigma}=T_{\sigma}(x), \quad \operatorname{order}(\sigma) \leq s .
$$


3. The coframe $\boldsymbol{\Theta}$ is fully regular if $\mathbf{T}^{(s)}$ is regular for all $s \geq 0$. In this case, let $\rho_{s}=$ $\operatorname{rank}\left(\mathbf{T}^{(s)}\right)$, and define the $\operatorname{rank}$ of $\boldsymbol{\Theta}$ as the minimal $s$ such that $\rho_{s}=\rho_{s+1}$.

4. The $s^{\text {th }}$ order classifying set is $\mathcal{C}^{(s)}(\boldsymbol{\Theta}, U)=\left\{\mathbf{T}^{(s)}(x): x \in U\right\} \subset \mathbb{K}^{(s)}$.

As a consequence of the invariance equations (4.4), if $\boldsymbol{\Theta}$ and $\overline{\boldsymbol{\Theta}}$ are equivalent coframes via $\Phi: U \rightarrow \bar{U}$, then

$$
\mathcal{C}^{(s)}(\boldsymbol{\Theta}, U)=\mathcal{C}^{(s)}(\overline{\mathbf{\Theta}}, \Phi(U)), \quad \forall s \geq 0 .
$$

This is sufficient in the fully regular case. We refer the reader to [22] for a proof of the following theorem.

Theorem 4.1. Suppose $\boldsymbol{\Theta}, \overline{\boldsymbol{\Theta}}$ are fully regular coframes on $U, \bar{U}$ respectively. There exists $\Phi$ satisfying $\Phi^{*} \overline{\boldsymbol{\Theta}}=\boldsymbol{\Theta}$ if and only if for each $s \geq 0, \mathcal{C}^{(s)}(\boldsymbol{\Theta}, U) \cap \mathcal{C}^{(s)}(\overline{\boldsymbol{\Theta}}, \bar{U})$ is nonempty. The set of self-equivalences $\Phi$ (i.e. satisfying $\Phi^{*} \boldsymbol{\Theta}=\boldsymbol{\Theta}$ ) defines a p-dimensional local Lie group of transformations, where $p=m-\operatorname{rank}(\boldsymbol{\Theta}) \geq 0$.

\section{Nine-dimensional maximal symmetry}

The solution to the Cartan equivalence problem (4.3) begins by lifting the problem to the left principal bundles $\Sigma_{7} \times G \stackrel{\pi}{\rightarrow} \Sigma_{7}$ and $\bar{\Sigma}_{7} \times G \stackrel{\bar{\pi}}{\rightarrow} \bar{\Sigma}_{7}$ by defining

$$
\left.\hat{\boldsymbol{\omega}}\right|_{(u, g)}=\left.g \pi^{*} \boldsymbol{\omega}\right|_{u},\left.\quad \hat{\overline{\boldsymbol{\omega}}}\right|_{(\bar{u}, g)}=\left.g \bar{\pi}^{*} \overline{\boldsymbol{\omega}}\right|_{\bar{u}}, \quad \text { where } \quad u \in \Sigma_{7}, \quad \bar{u} \in \bar{\Sigma}_{7}, \quad g \in G,
$$

and noting the following key lemma [10].

Lemma 5.1. There exists an equivalence $\phi$ as in (4.3) if and only if there exists a local diffeomorphism $\Phi: \Sigma_{7} \times G \rightarrow \bar{\Sigma}_{7} \times G$ satisfying $\Phi^{*} \hat{\overline{\boldsymbol{\omega}}}=\hat{\boldsymbol{\omega}}$.

Identifying the coframe $\boldsymbol{\omega}$ on $\Sigma_{7}$ with its pullback by the canonical projection $\Sigma_{7} \times G \rightarrow \Sigma_{7}$, we can write

$$
\hat{\omega}^{i}=g_{j}^{i} \omega^{j}, \quad g \in G .
$$

Using (2.8), the structure equations for these lifted forms are then

$$
d \hat{\omega}^{i}=\left(d g \cdot g^{-1}\right)^{i}{ }_{j} \wedge \hat{\omega}^{j}+\frac{1}{2} \hat{\gamma}^{j}{ }_{k \ell} \hat{\omega}^{k} \wedge \hat{\omega}^{\ell},
$$

where the coefficients $\gamma^{i}{ }_{j k}$ transform tensorially under the $G$-action

$$
\hat{\gamma}_{j k}^{i}:=g^{i}{ }_{\ell} \gamma_{m n}\left(g^{-1}\right)^{m}{ }_{j}\left(g^{-1}\right)^{n}{ }_{k},
$$

and $d g \cdot g^{-1}$ refers to the right-invariant Maurer-Cartan form on $G$. Since $D_{8}$ is discrete, then if $(g, k) \in G^{0} \times D_{8}$

$$
d(g k) \cdot(g k)^{-1}=d g \cdot k \cdot k^{-1} g^{-1}=d g \cdot g^{-1},
$$

and so we can identify the Maurer-Cartan form on $G$ with that on $G^{0}$. For $g=M\left(a_{1}, a_{2}, a_{3}\right) \in$ $G^{0}$ as in (4.2), we have $g^{-1}=M\left(\frac{1}{a_{1}},-\frac{a_{2}}{a_{1}},-\frac{a_{3}}{a_{1}}\right)$ and

$$
d g \cdot g^{-1}=\left(\begin{array}{ccccccc}
2 \alpha^{1} & 0 & 0 & 0 & 0 & 0 & 0 \\
\alpha^{2} & \alpha^{1} & 0 & 0 & 0 & 0 & 0 \\
\epsilon \alpha^{3} & 0 & \alpha^{1} & 0 & 0 & 0 & 0 \\
0 & 0 & 0 & \alpha^{1} & 0 & 0 & 0 \\
0 & 0 & 0 & \alpha^{3} & 0 & 0 & 0 \\
0 & 0 & 0 & 0 & 0 & \alpha^{1} & 0 \\
0 & 0 & 0 & 0 & 0 & \alpha^{2} & 0
\end{array}\right),
$$


where

$$
\alpha^{1}=\frac{d a_{1}}{a_{1}}, \quad \alpha^{2}=\frac{d a_{2}}{a_{1}}, \quad \alpha^{3}=\frac{d a_{3}}{a_{1}}
$$

are a basis for the right-invariant 1-forms on $G^{0}$ and hence $G$. Identifying $\alpha^{i}$ on $G$ with their pullback by the canonical projection $\Sigma_{7} \times G \rightarrow G$, we have the structure equations for the lifted coframe:

$$
\begin{aligned}
& d \hat{\omega}^{1}=2 \alpha^{1} \wedge \hat{\omega}^{1}+\hat{\omega}^{3} \wedge \hat{\omega}^{6}+\hat{\omega}^{2} \wedge \hat{\omega}^{4}+\eta_{1} \wedge \hat{\omega}^{1}, \\
& d \hat{\omega}^{2}=\alpha^{2} \wedge \hat{\omega}^{1}+\alpha^{1} \wedge \hat{\omega}^{2}+\hat{\omega}^{4} \wedge \hat{\omega}^{5}+\hat{\omega}^{3} \wedge \hat{\omega}^{7}+\eta_{2} \wedge \hat{\omega}^{1}+\eta_{3} \wedge \hat{\omega}^{2}, \\
& d \hat{\omega}^{3}=\epsilon \alpha^{3} \wedge \hat{\omega}^{1}+\alpha^{1} \wedge \hat{\omega}^{3}+\hat{\omega}^{6} \wedge \hat{\omega}^{7}+\epsilon \hat{\omega}^{2} \wedge \hat{\omega}^{5}+\eta_{4} \wedge \hat{\omega}^{1}+\eta_{5} \wedge \hat{\omega}^{3}, \\
& d \hat{\omega}^{4}=\alpha^{1} \wedge \hat{\omega}^{4}+\eta_{6} \wedge \hat{\omega}^{1}+\eta_{7} \wedge \hat{\omega}^{2}+\eta_{8} \wedge \hat{\omega}^{4}+\eta_{9} \wedge \hat{\omega}^{5}, \\
& d \hat{\omega}^{5}=\alpha^{3} \wedge \hat{\omega}^{4}+\eta_{10} \wedge \hat{\omega}^{1}+\eta_{11} \wedge \hat{\omega}^{2}+\eta_{12} \wedge \hat{\omega}^{4}+\eta_{13} \wedge \hat{\omega}^{5}, \\
& d \hat{\omega}^{6}=\alpha^{1} \wedge \hat{\omega}^{6}+\eta_{14} \wedge \hat{\omega}^{1}+\eta_{15} \wedge \hat{\omega}^{3}+\eta_{16} \wedge \hat{\omega}^{6}+\eta_{17} \wedge \hat{\omega}^{7}, \\
& d \hat{\omega}^{7}=\alpha^{2} \wedge \hat{\omega}^{6}+\eta_{18} \wedge \hat{\omega}^{1}+\eta_{19} \wedge \hat{\omega}^{3}+\eta_{20} \wedge \hat{\omega}^{6}+\eta_{21} \wedge \hat{\omega}^{7}, \\
& d \alpha^{1}=0 \\
& d \alpha^{2}=-\alpha^{1} \wedge \alpha^{2}, \\
& d \alpha^{3}=-\alpha^{1} \wedge \alpha^{3},
\end{aligned}
$$

where $\eta_{i}$ are semi-basic 1 -forms with respect to the projection $\Sigma_{7} \times G \rightarrow \Sigma_{7}$. The structure equations for the lifted forms $\hat{\omega}^{i}$ can be written

$$
d \hat{\omega}^{i}=a_{\rho j}^{i} \alpha^{\rho} \wedge \hat{\omega}^{j}+\frac{1}{2} \hat{\gamma}^{i}{ }_{j k} \hat{\omega}^{j} \wedge \hat{\omega}^{k}
$$

where $a_{\rho j}^{i}$ are constants (c.f. Maurer-Cartan form) and $\hat{\gamma}_{j k}^{i}$ is defined as in (5.1).

Definition 5.1. The degree of indeterminacy $r^{(1)}$ of a lifted coframe is the number of free variables in the set of transformations $\alpha^{\rho} \mapsto \alpha^{\rho}+\lambda^{\rho}{ }_{i} \hat{\omega}^{i}$ which preserve the structure equations for $d \hat{\omega}^{i}$.

For later use, we note the following:

Lemma 5.2. For our lifted coframe $\boldsymbol{\Theta}=\{\hat{\boldsymbol{\omega}}, \boldsymbol{\alpha}\}$ on $\Sigma_{7} \times G$ satisfying $(5.2)$, we have $r^{(1)}=0$.

Proof. From the $d \hat{\omega}^{1}, d \hat{\omega}^{2}, d \hat{\omega}^{3}$ equation in (5.2), we must have

$$
\alpha^{1} \mapsto \alpha^{1}+\lambda \hat{\omega}^{1}, \quad \alpha^{2} \mapsto \alpha^{2}+\lambda \hat{\omega}^{2}, \quad \alpha^{3} \mapsto \alpha^{3}+\epsilon \lambda \hat{\omega}^{3} .
$$

However, to preserve the form of $d \hat{\omega}^{i}, i=4,5,6,7$, we must have $\lambda=0$. Since there are no free variables, then $r^{(1)}=0$.

The goal in Cartan's solution algorithm is to reduce to an $\{e\}$-structure so that Theorem 4.1 can be invoked. This amounts to essentially adapting the coframes on the base, i.e. fixing a map $g: \Sigma_{7} \rightarrow G$. Using Lemma 5.1, coefficients in the structure equations are candidates for normalization, from which the structure group $G$ can be subsequently reduced. However, we only use those coefficients which are not affected by the choice of any map $g: \Sigma_{7} \rightarrow G$. Note that pulling the Maurer-Cartan forms back to the base by such a map will express each $\alpha^{\rho}$ in terms of the new coframe $\hat{\boldsymbol{\omega}}$ (pulled back to the base). This motivates the following definition. 
Definition 5.2. Given a lifted coframe, Lie algebra valued compatible absorption refers to redefining the right-invariant 1-forms $\alpha^{\rho}$ by $\hat{\alpha}^{\rho}=\alpha^{\rho}+\lambda^{\rho}{ }_{i} \hat{\omega}^{i}$, where $\lambda^{\rho}{ }_{i}$ are functions on the bundle. The terms involving the coefficients $\hat{\gamma}^{i}{ }_{j k}$ which cannot be eliminated by means of Lie algebra valued compatible absorption are called torsion terms and the corresponding coefficients are referred to as torsion coefficients.

From (5.2), the $d \hat{\omega}^{5}$ and $d \hat{\omega}^{7}$ structure equations indicate that $\hat{\gamma}^{5}{ }_{56}, \hat{\gamma}^{5}{ }_{57}, \hat{\gamma}^{7}{ }_{47}, \hat{\gamma}^{7}{ }_{57}$ are torsion coefficients. Using (3.2), (3.4), and the tensor transformation law (5.1) for the $\gamma^{\prime}$ 's, we see that there is a well-defined $G$-action on $\mathbb{R}^{4}$ (i.e. the range of $\left.\left(\gamma_{56}^{5}, \gamma^{5}{ }_{57}, \gamma^{7}{ }_{47}, \gamma^{7}{ }_{57}\right)\right)$ given by the formulas

\begin{tabular}{|l|c|c|c|}
\hline & $G^{0}$-action by $g=M\left(a_{1}, a_{2}, a_{3}\right)$ & $R$-action & $S$-action \\
\hline$\hat{\gamma}^{5}{ }_{56}$ & $\frac{1}{a_{1}}\left(\gamma^{5}{ }_{56}-\gamma^{5}{ }_{57} a_{2}+a_{3} \epsilon\right)$ & $-\gamma^{7}{ }_{47}$ & $\gamma^{5}{ }_{56}$ \\
$\hat{\gamma}^{7}{ }_{47}$ & $\frac{1}{a_{1}}\left(\gamma^{7}{ }_{47}-a_{2}-\gamma^{7}{ }_{57} a_{3}\right)$ & $\gamma^{5}{ }_{56}$ & $-\gamma^{7}{ }_{47}$ \\
$\hat{\gamma}^{5}{ }_{57}$ & $\gamma^{5}{ }_{57}$ & $\epsilon \gamma^{7}{ }_{57}$ & $-\gamma^{5}{ }_{57}$ \\
$\hat{\gamma}^{7}{ }_{57}$ & $\gamma^{7}{ }_{57}$ & $\epsilon \gamma^{5}{ }_{57}$ & $-\gamma^{7}{ }_{57}$ \\
\hline
\end{tabular}

We can always normalize $\hat{\gamma}^{5}{ }_{56}$ to zero by using the $G^{0}$-action and setting

$$
a_{3}=\epsilon\left(-\gamma^{5}{ }_{56}+\gamma^{5}{ }_{57} a_{2}\right) \text {. }
$$

The matrix factorization

$$
M\left(a_{1}, a_{2}, \epsilon\left(-\gamma^{5}{ }_{56}+\gamma^{5}{ }_{57} a_{2}\right)\right)=M\left(a_{1}, a_{2}, \epsilon \gamma^{5}{ }_{57} a_{2}\right) M\left(1,0,-\epsilon \gamma_{56}^{5}\right)
$$

indicates that we can normalize $\gamma^{5}{ }_{56}$ to 0 for the base coframe via

$$
\bar{\omega}^{3}=-\gamma^{5}{ }_{56} \omega^{1}+\omega^{3}, \quad \bar{\omega}^{5}=-\epsilon \gamma^{5}{ }_{56} \omega^{4}+\omega^{5} .
$$

This change of coframe is admissible in the sense that it preserves the form of the structure equations in (3.2) and (3.4). (We henceforth drop the bars.) Thus, we have the normal form $\Gamma=\left(\gamma_{56}^{5}=0, \gamma_{57}^{5}, \gamma^{7}{ }_{47}, \gamma^{7}{ }_{57}\right)$. In general, however, this is a normalization of nonconstant type since $\Gamma$ still may depend on $x \in \Sigma_{7}$. Pointwise, we define the reduced structure group $G_{\Gamma}$ as the stabilizer of $\Gamma$, i.e. it is the subgroup of $G$ preserving the structure equations together with the normalization given by $\Gamma$. Clearly, the 1-parameter subgroup generated $M\left(1,0, a_{3}\right)\left(a_{3} \in \mathbb{R}\right)$ yields a 1-dimensional orbit through $\Gamma$ and so $\operatorname{dim}\left(G_{\Gamma}\right) \leq 2 \operatorname{since} \operatorname{dim}(G)=3$.

The algorithm continues by means of further normalizations and reductions of the structure group until one of two possibilities occurs:

1) the structure group has been reduced to the identity, i.e. get an $\{e\}$-structure on $\Sigma_{7}$, or

2) the structure group has not been reduced to the identity but the structure group acts trivially on the torsion coefficients.

By Theorem 4.1, the former possibility yields a symmetry group of dimension at most seven. In the latter case, the next step in the algorithm is to prolong the problem to the space $\Sigma_{7} \times G_{\Gamma}$. Here, we have abused notation and written $G_{\Gamma}$ also for the structure group in the latter possibility above. Since, by Lemma $5.2, r^{(1)}=0$ with respect to the lifted coframe on $\Sigma \times G$, it is clear that we must have $r^{(1)}=0$ for the lifted coframe on $\Sigma_{7} \times G_{\Gamma}$. Finally, we invoke the following standard theorem (Proposition 12.1 in [22]) written here in our notation:

Proposition 5.1. Let $\hat{\boldsymbol{\omega}}, \hat{\overline{\boldsymbol{\omega}}}$ be lifts of coframes $\boldsymbol{\omega}, \overline{\boldsymbol{\omega}}$ having the same structure group $G_{\Gamma}$, no group dependent torsion coefficients, and $r^{(1)}=0$. Let $\hat{\boldsymbol{\alpha}}$, $\hat{\overline{\boldsymbol{\alpha}}}$ be modified Maurer-Cartan forms obtained by performing a full Lie algebra-valued compatible absorption. Denote $\boldsymbol{\Theta}=\{\hat{\boldsymbol{\omega}}, \hat{\boldsymbol{\alpha}}\}$, $\overline{\boldsymbol{\Theta}}=\{\hat{\overline{\boldsymbol{\omega}}}, \hat{\overline{\boldsymbol{\alpha}}}\}$. Then there exists $\phi: \Sigma_{7} \rightarrow \bar{\Sigma}_{7}$ satisfying $\phi^{*} \overline{\boldsymbol{\omega}}=g \boldsymbol{\omega}$ for some $g: \Sigma_{7} \rightarrow G_{\Gamma}$ if and only if there exists $\Phi: \Sigma_{7} \times G_{\Gamma} \rightarrow \bar{\Sigma}_{7} \times G_{\Gamma}$ satisfying $\Phi^{*} \overline{\boldsymbol{\Theta}}=\mathbf{\Theta}$. 
In other words, we have prolonged to an $\{e\}$-structure on $\Sigma_{7} \times G_{\Gamma}$. Since $\operatorname{dim}\left(G_{\Gamma}\right) \leq 2$ for any choice of $\Gamma$, then the symmetry group of the coframe is at most nine-dimensional. Thus, we have proven:

Theorem 5.1. The (contact) symmetry group of any generic hyperbolic equation is finite dimensional and has maximal dimension 9.

In fact, this upper bound is sharp. We will give explicit normal forms for all contactequivalence classes of generic hyperbolic equations with 9-dimensional symmetry along with their corresponding symmetry generators and corresponding structure equations.

Define

$$
m:=\gamma^{5}{ }_{57} \in C^{\infty}\left(\Sigma_{7}\right), \quad n:=\gamma^{7}{ }_{57} \in C^{\infty}\left(\Sigma_{7}\right),
$$

and note that although $m$ and $n$ are $G^{0}$-invariant, they are not $G$-invariant. However, along each $G$-orbit the product $m n$ is invariant.

We define two functions which will play an important role in the classifications to follow. Define

$$
\Delta_{1}=m n+\epsilon, \quad \Delta_{2}=m^{2}-\epsilon n^{2}
$$

Note that $\Delta_{1}$ is a contact invariant, and $\Delta_{2}$ is a relative contact invariant: it is $G^{+}$-invariant, but under the $R$-action, $\hat{\Delta}_{2}=-\epsilon \Delta_{2}$.

Corollary 5.1. If a generic hyperbolic equation has 9-dimensional symmetry group, then $\Delta_{1}=0$.

Proof. Under the assumption of maximal symmetry, all torsion coefficients must be constant. Thus, $\hat{m}, \hat{n}$ and consequently $m, n$ must be constant. If $\Delta_{1} \neq 0$, then there is a unique solution to the linear system

$$
\left(\begin{array}{cc}
m & -\epsilon \\
1 & n
\end{array}\right)\left(\begin{array}{l}
a_{2} \\
a_{3}
\end{array}\right)=\left(\begin{array}{l}
\gamma^{5} 56 \\
\gamma^{7}{ }_{47}
\end{array}\right),
$$

which yields the normalizations $\hat{\gamma}_{56}^{5}=\hat{\gamma}_{47}^{7}=0$ and a two dimensional reduction of the initial structure group $G$. Consequently, the stabilizer $G_{\Gamma}$ would be at most 1-dimensional and the symmetry group would be at most 8-dimensional. Thus, we must have $\Delta_{1}=0$.

\section{Complete structure equations}

In Appendix D, we provide details of Vranceanu's reduction of the generic hyperbolic structure equations which allowed him to isolate the maximally symmetric and two sets of submaximally symmetric structures.

Theorem 6.1. Let $K^{0}=\left\{\operatorname{diag}\left(a_{1}^{2}, a_{1}, a_{1}, a_{1}, 1, a_{1}, 1\right): a_{1}>0\right\} \subset G$. Consider a coframe $\left\{\omega^{i}\right\}_{i=1}^{7}$ on $\Sigma_{7}$ satisfying the generic hyperbolic structure equations (3.2) and (3.4), and the corresponding lifted coframe on $\Sigma_{7} \times K^{0} \rightarrow \Sigma_{7}$. If:

1) all torsion coefficients on which $K^{0}$ acts nontrivially are constants, and

2) $K^{0}$ cannot be reduced to the identity,

then the structure equations can be put in the form

$$
d \omega^{1}=\omega^{3} \wedge \omega^{6}+\omega^{2} \wedge \omega^{4}
$$




$$
\begin{aligned}
& d \omega^{2}=\omega^{4} \wedge \omega^{5}+\omega^{3} \wedge \omega^{7}+\omega^{2} \wedge\left(-\frac{3 n}{2} \omega^{5}+\frac{m}{2} \omega^{7}\right), \\
& d \omega^{3}=\omega^{6} \wedge \omega^{7}+\epsilon \omega^{2} \wedge \omega^{5}+\omega^{3} \wedge\left(-\frac{n}{2} \omega^{5}+\frac{3 m}{2} \omega^{7}\right), \\
& d \omega^{4}=\epsilon \omega^{5} \wedge \omega^{6}+\omega^{2} \wedge\left(B \omega^{5}+\gamma^{4}{ }_{27} \omega^{7}\right)+\omega^{4} \wedge\left(\frac{3 n}{2} \omega^{5}-\frac{m}{2} \omega^{7}\right), \\
& d \omega^{5}=m \omega^{5} \wedge \omega^{7}, \\
& d \omega^{6}=-\omega^{4} \wedge \omega^{7}+\omega^{3} \wedge\left(\gamma^{6}{ }_{35} \omega^{5}+\epsilon B \omega^{7}\right)+\omega^{6} \wedge\left(\frac{n}{2} \omega^{5}-\frac{3 m}{2} \omega^{7}\right), \\
& d \omega^{7}=n \omega^{5} \wedge \omega^{7},
\end{aligned}
$$

where $m, n, B \in C^{\infty}\left(\Sigma_{7}\right)$,

$$
\begin{aligned}
d m= & m_{5} \omega^{5}+m_{7} \omega^{7}, \quad d n=n_{5} \omega^{5}+n_{7} \omega^{7}, \quad m_{57}=\frac{\partial m_{5}}{\partial \omega^{7}}=\frac{\partial}{\partial \omega^{7}}\left(\frac{\partial m}{\partial \omega^{5}}\right), \quad \text { etc. } \\
d B= & \epsilon\left(-4 m \Delta_{1}-2 n \epsilon B-6 m m_{5}-m n_{7}+n m_{7}+\frac{3}{2} m_{57}+\frac{1}{2} n_{77}\right) \omega^{5} \\
& +\left(4 n \Delta_{1}+2 m B-6 n n_{7}-n m_{5}+m n_{5}-\frac{1}{2} m_{55}-\frac{3}{2} n_{75}\right) \omega^{7}
\end{aligned}
$$

and

$$
\gamma^{4}{ }_{27}=m n+\epsilon-\frac{3}{2} n_{7}-\frac{1}{2} m_{5}, \quad \gamma^{6}{ }_{35}=m n+\epsilon+\frac{1}{2} n_{7}+\frac{3}{2} m_{5} .
$$

Finally, the integrability conditions for (6.1) (i.e. $d^{2} \omega^{i}=0$ for all $i$ ) reduce to the integrability conditions for $d m, d n, d B$ as given above.

Remark 6.1. All structures admitting a 9-dimensional symmetry group are included in (6.1) (since $K^{0}$ cannot be reduced to the identity).

Remark 6.2. For all valid structures arising from (6.1), the function $\Delta_{3}=B:=\gamma^{4}{ }_{25}$ is a relative contact invariant: it is $G^{+}$-invariant, and under the $R$-action, $\hat{\Delta}_{3}=\epsilon \Delta_{3}$.

Corollary 6.1. For all valid structures arising from (6.1), the original $G$-structure on $\Sigma_{7}$ can be reduced to an $H$-structure, where $H=H^{0} \rtimes D_{8}$ and

$$
H^{0}=\left\{\left(\begin{array}{ccccccc}
a_{1}{ }^{2} & 0 & 0 & 0 & 0 & 0 & 0 \\
a_{1} a_{2} & a_{1} & 0 & 0 & 0 & 0 & 0 \\
m a_{1} a_{2} & 0 & a_{1} & 0 & 0 & 0 & 0 \\
0 & 0 & 0 & a_{1} & 0 & 0 & 0 \\
0 & 0 & 0 & \epsilon m a_{2} & 1 & 0 & 0 \\
0 & 0 & 0 & 0 & 0 & a_{1} & 0 \\
0 & 0 & 0 & 0 & 0 & a_{2} & 1
\end{array}\right):\left(a_{1}, a_{2}\right) \in \mathbb{R}^{+} \times \mathbb{R}\right\}
$$

Moreover, wherever $\Delta_{1} \neq 0$, or $B \neq 0$ there is a further reduction to a $K$-structure, where $K=K^{0} \rtimes D_{8}$.

Proof. For all valid structures satisfying (6.1), $\gamma^{5}{ }_{56}=\gamma^{7}{ }_{47}=0$, so from the $G$-action described in (5.4), the stabilizer $G_{\Gamma}$ of $\Gamma=\left(\gamma_{56}^{5}, \gamma^{7}{ }_{47}, \gamma^{5}{ }_{57}, \gamma^{7}{ }_{57}\right)=(0,0, m, n)$ is contained in $H$ (since we can always keep $\hat{\gamma}_{56}^{5}=0$ using $\left.a_{3}=\epsilon m a_{2}\right)$. 
If $\Delta_{1} \neq 0$, then $a_{2}=a_{3}=0$ is the unique solution to (5.6) and $G_{\Gamma} \subset K$. Alternatively, suppose $B \neq 0$. Note that $\hat{\gamma}^{4}{ }_{15}$ and $\hat{\gamma}^{6}{ }_{17}$ are torsion coefficients, and for the structure equations (6.1), we have $\gamma^{4}{ }_{15}=\gamma^{6}{ }_{17}=0$, and the transformation laws (under $H^{0}$ ):

$$
\hat{\gamma}^{4}{ }_{15}=\frac{-B a_{2}}{a_{1}}, \quad \hat{\gamma}^{6}{ }_{17}=\frac{-B \epsilon m a_{2}}{a_{1}} .
$$

Consequently, we can normalize $\hat{\gamma}^{4}{ }_{15}=\hat{\gamma}^{6}{ }_{17}=0$ and reduce the connected component of the structure group to $K^{0}$ by setting $a_{2}=0$. The discrete part of the structure group will preserve this reduction since

$$
\begin{array}{lll}
R \text {-action }: & \hat{\gamma}^{4}{ }_{15}=-\gamma^{6}{ }_{17}, & \hat{\gamma}^{6}{ }_{17}=\gamma^{4}{ }_{15}, \\
S \text {-action }: & \hat{\gamma}^{4}{ }_{15}=-\gamma^{4}{ }_{15}, & \hat{\gamma}^{6}{ }_{17}=\gamma^{6}{ }_{17} .
\end{array}
$$

Let us now examine in detail the case when $m, n$ are constants. Then (6.2) becomes

$$
d B=-2\left(2 \epsilon m \Delta_{1}+n B\right) \omega^{5}+2\left(2 n \Delta_{1}+m B\right) \omega^{7} .
$$

Applying $d$ to (6.4) and simplifying, we obtain the integrability condition

$$
0=-12 \epsilon \Delta_{1} \Delta_{2} \omega^{5} \wedge \omega^{7}
$$

Corollary 6.2. Suppose $m, n$ are constants. Then $\Delta_{1} \Delta_{2}=0$ if and only if (6.1) are valid structure equations. Moreover, in this case:

1) $\sigma=-n \omega^{5}+m \omega^{7}$ is closed, so $\sigma=d h$ for some function $h \in C^{\infty}\left(\Sigma_{7}\right)$;

2) $m=0$ iff $n=0$ iff $\sigma=0$ iff $h$ is constant;

3) if $\Delta_{1}=0$, then $n=-\frac{\epsilon}{m}$, and $d B=2 B \sigma$, so $B=b e^{2 h}$, where $b$ is an arbitrary constant;

4) if $\Delta_{2}=0$, then:

- if $\epsilon=-1$, then $m=n=0$, and $B$ is an arbitrary constant;

- if $\epsilon=1$, then letting $n=\epsilon_{1} m, \epsilon_{1}= \pm 1$ we have $d B=2\left(2\left(m^{2}+\epsilon_{1}\right)+B\right) \sigma$. If $m \neq 0$, then $B=-2\left(m^{2}+\epsilon_{1}\right)+b e^{2 h}$, where $b$ is an arbitrary constant. If $m=0$, then $B$ is an arbitrary constant;

5) If $\Delta_{1}=\Delta_{2}=0$, then $\epsilon=1$, and $(m, n)=(1,-1)$ or $(-1,1)$.

All of the above structures have a symmetry group with dimension at least seven.

Proof. We prove only the final assertion as the others are straightforward to prove. Let $G_{\Gamma}$ be the reduced structure group for which there is no group dependent torsion. By construction (c.f. Theorem 6.1), we must have $K^{0} \subset G_{\Gamma}$, and by Proposition 5.1 we prolong to an $\{e\}$ structure on $\Sigma_{7} \times G_{\Gamma}$. If $B$ is constant, then by Theorem 4.1 the symmetry group has dimension $\operatorname{dim}\left(\Sigma_{7} \times G_{\Gamma}\right) \geq 8$. If $B$ is nonconstant, then by Corollary $6.1, G_{\Gamma} \subset K$. Note that $\hat{B}=B$, so equation (6.4) implies that on $\Sigma_{7} \times G_{\Gamma}$, we have

$$
d B=-2\left(2 \epsilon m \Delta_{1}+n B\right) \hat{\omega}^{5}+2\left(2 n \Delta_{1}+m B\right) \hat{\omega}^{7} .
$$

Thus, the coframe derivatives of $B$ are functions of $B$. Thus, if $B$ is nonconstant, then the rank of the lifted coframe $\boldsymbol{\Theta}$ is 1 and by Theorem 4.1 the symmetry group will be at least $\operatorname{dim}\left(\Sigma_{7} \times G_{\Gamma}\right)-\operatorname{rank}(\boldsymbol{\Theta}) \geq 8-1=7$ dimensional.

Remark 6.3. In the case $\Delta_{2}=0, \epsilon=1$, we note that $\epsilon_{1}$ is a contact invariant. 
Certain values of $m, n, B$ lead to equivalent structures owing to the presence of the $D_{8}$ discrete subgroup of the original structure group $G$. Suppose $\Delta_{1}=0$, so $n=-\frac{\epsilon}{m}$. Then

$$
\begin{array}{ll}
R \text {-action }: & \hat{m}=-\frac{1}{m}, \quad \hat{B}=\epsilon B, \\
S \text {-action }: & \hat{m}=-m, \quad \hat{B}=B .
\end{array}
$$

In this case, by choosing a representative element $m \in(0,1]$, we can reduce $D_{8}$ to $\mathbb{Z}_{2}=\left\langle R^{2}\right\rangle$. If $\epsilon=1$, no further reduction occurs. If $\epsilon=-1$ and $B \neq 0$, we choose a representative out of $\{B,-B\}$ to reduce the discrete subgroup to the identity. A similar argument is used in the case $\Delta_{1} \neq 0$, where $\Delta_{2}=0, n=\epsilon_{1} m$, and

$$
\begin{array}{ll}
R \text {-action }: & \hat{m}=\epsilon \epsilon_{1} m, \quad \hat{B}=\epsilon B, \\
S \text {-action }: & \hat{m}=-m, \quad \hat{B}=B .
\end{array}
$$

\begin{tabular}{|c|c|c|c|c|c|c|}
\hline Sym. grp. & $\Delta_{1}$ & $\Delta_{2}$ & $(\epsilon, m)$ & $n$ & $B$ & Str. grp. $G_{\Gamma}$ \\
\hline 9 & 0 & $\neq 0$ & $\begin{array}{l}\{ \pm 1\} \times(0,1] \\
\text { except }(1,1)\end{array}$ & $-\frac{\epsilon}{m}$ & 0 & $H^{0} \rtimes\left\langle R^{2}\right\rangle$ \\
\hline 9 & 0 & 0 & $(1,1)$ & -1 & 0 & $H^{0} \rtimes\left\langle R^{2}\right\rangle$ \\
\hline 8 & $\neq 0$ & 0 & $(-1,0)$ & 0 & $b>0$ & $K^{0} \rtimes\left\langle R^{2}, S\right\rangle$ \\
\hline 8 & $\neq 0$ & 0 & $(-1,0)$ & 0 & 0 & $K^{0} \rtimes D_{8}$ \\
\hline 8 & $\neq 0$ & 0 & $(1,0)$ & 0 & $b \in \mathbb{R}$ & $K^{0} \rtimes D_{8}$ \\
\hline 8 & $\neq 0$ & 0 & $\{1\} \times(0, \infty)$ & $m$ & $-2\left(m^{2}+1\right)$ & $K^{0} \rtimes\langle R\rangle$ \\
\hline 8 & $\neq 0$ & 0 & $\{1\} \times(0, \infty)$ & $-m$ & $-2\left(m^{2}-1\right)$ & $K^{0} \rtimes\left\langle R^{2}\right\rangle$ \\
\hline 7 & 0 & $\neq 0$ & $\{-1\} \times(0,1]$ & $\frac{1}{m}$ & $b e^{2 h}, b>0$ & $K^{0}$ \\
\hline 7 & 0 & $\neq 0$ & $\{1\} \times(0,1)$ & $-\frac{1}{m}$ & $b e^{2 h}, b \in \mathbb{R}^{\times}$ & $K^{0} \rtimes\left\langle R^{2}\right\rangle$ \\
\hline 7 & 0 & 0 & $(1,1)$ & -1 & $b e^{2 h}, b \in \mathbb{R}^{\times}$ & $K^{0} \rtimes\left\langle R^{2}\right\rangle$ \\
\hline 7 & $\neq 0$ & 0 & $\{1\} \times(0, \infty)$ & $m$ & $-2\left(m^{2}+1\right)+b e^{2 h}, b \in \mathbb{R}$ & $K^{0} \rtimes\langle R\rangle$ \\
\hline 7 & $\neq 0$ & 0 & $\{1\} \times(0, \infty)$ & $-m$ & $-2\left(m^{2}-1\right)+b e^{2 h}, b \in \mathbb{R}$ & $K^{0} \rtimes\left\langle R^{2}\right\rangle$ \\
\hline
\end{tabular}

The results are organized in Table 4 according to the dimension of the symmetry group of the resulting $\{e\}$-structures on $\Sigma_{7} \times G_{\Gamma}$.

Table 4. All generic hyperbolic structures for which $m, n$ are constants and $K^{0} \subset G_{\Gamma}$.

( $h$ is a nonconstant function such that $d h=-n \omega^{5}+m \omega^{7}$ )

Remark 6.4. Vranceanu explicitly derived the following constant torsion cases:

- 9-dim. symmetry: $\epsilon=1, \Delta_{1}=0, B=0$;

- 8-dim. symmetry:

1) $\epsilon=1, \Delta_{1} \neq 0, \Delta_{2}=0, m=n=0$,

2) $\epsilon=1, \Delta_{1} \neq 0, \Delta_{2}=0, n= \pm m, B=-2\left(m^{2} \pm 1\right)$.

Theorem 6.2. All contact-inequivalent generic hyperbolic structures for which:

1) $K^{0}$ is a subgroup of the structure group, and

2) $m, n$ are constants,

are displayed in Table 4. 
For ease of reference, we state below the structure equations explicitly for each of the cases above. For the maximally symmetric cases, we state the structure equations for both the base coframe $\left\{\omega^{1}, \ldots, \omega^{7}\right\}$ and the lifted coframe on $\Sigma_{7} \times G_{\Gamma}$. In the submaximally symmetric cases, we only display structure equations for the lifted coframe. (One can obtain the structure equations on the base simply by setting $\hat{\alpha}^{1}=0$ and removing all hats from the remaining variables.) In each case, we assume that $G_{\Gamma}$ and all parameters are as in Table 4 . Note that $d \hat{\omega}^{i}$ are determined by (5.3). Following potentially some Lie algebra valued compatible absorption, $\hat{\alpha}^{\rho}=\alpha^{\rho}+\lambda^{\rho} i^{i}$, the structure equations $d \hat{\alpha}^{\rho}$ are determined by the integrability conditions $d^{2} \hat{\omega}^{i}=0$. (We only display the final results.)

For those coframes whose structure equations depend explicitly on the (nonconstant) function $h$, we have $m \neq 0$ (c.f. Table 4 ) and the symmetry algebra is determined by restricting to the level set $h=h_{0}$, where $h_{0}$ is a constant. (Note: We will abuse notation and identify $h \in C^{\infty}\left(\Sigma_{7}\right)$ with its pullback to the bundle.) On this level set, we have $0=d h=-n \hat{\omega}^{5}+m \hat{\omega}^{7}$. We can choose (the pullback of) $\left\{\hat{\omega}^{1}, \ldots, \hat{\omega}^{6}, \hat{\alpha}^{1}\right\}$ as a coframe on each level set, and the corresponding structure equations will have constant coefficients. Thus, these are Maurer-Cartan equations for a local Lie group. A well-known fact is that the isomorphism type of the symmetry algebra of a coframe determined in this way is independent of the level set chosen. Consequently, we make the canonical choice and restrict to the level set $h=0$ in these cases.

The structure constants for the (contact) symmetry algebra for each of the structures can be read off from the structure equations for the coframe (or its pullback to the level set $h=0$ if $h$ appears explicitly). Only the symmetry algebras appearing in the 9-dimensional case will be studied in further detail in this article.

\subsection{Case 1: $\Delta_{1}=0, B=0$}

This branch consists of precisely all maximally symmetric generic hyperbolic equations.

Parameters: $\quad(\epsilon, m) \in\{ \pm 1\} \times(0,1]$.

Base coframe:

$$
\begin{aligned}
& d \omega^{1}=\omega^{2} \wedge \omega^{4}+\omega^{3} \wedge \omega^{6} \\
& d \omega^{2}=\omega^{4} \wedge \omega^{5}+\omega^{3} \wedge \omega^{7}+\omega^{2} \wedge\left(\frac{3 \epsilon}{2 m} \omega^{5}+\frac{m}{2} \omega^{7}\right), \\
& d \omega^{3}=\omega^{6} \wedge \omega^{7}+\epsilon \omega^{2} \wedge \omega^{5}+\omega^{3} \wedge\left(\frac{\epsilon}{2 m} \omega^{5}+\frac{3 m}{2} \omega^{7}\right), \\
& d \omega^{4}=\epsilon \omega^{5} \wedge \omega^{6}-\omega^{4} \wedge\left(\frac{3 \epsilon}{2 m} \omega^{5}+\frac{m}{2} \omega^{7}\right), \\
& d \omega^{5}=m \omega^{5} \wedge \omega^{7}, \\
& d \omega^{6}=-\omega^{4} \wedge \omega^{7}-\omega^{6} \wedge\left(\frac{\epsilon}{2 m} \omega^{5}+\frac{3 m}{2} \omega^{7}\right), \\
& d \omega^{7}=-\frac{\epsilon}{m} \omega^{5} \wedge \omega^{7} .
\end{aligned}
$$

Lifted coframe on $\Sigma_{7} \times G_{\Gamma}$ :

$$
\begin{aligned}
& d \hat{\omega}^{1}=2 \hat{\alpha}^{1} \wedge \hat{\omega}^{1}+\hat{\omega}^{2} \wedge \hat{\omega}^{4}+\hat{\omega}^{3} \wedge \hat{\omega}^{6}, \\
& d \hat{\omega}^{2}=\hat{\alpha}^{2} \wedge \hat{\omega}^{1}+\hat{\alpha}^{1} \wedge \hat{\omega}^{2}+\hat{\omega}^{4} \wedge \hat{\omega}^{5}+\hat{\omega}^{3} \wedge \hat{\omega}^{7}+\hat{\omega}^{2} \wedge\left(\frac{3 \epsilon}{2 m} \hat{\omega}^{5}+\frac{m}{2} \hat{\omega}^{7}\right), \\
& d \hat{\omega}^{3}=m \hat{\alpha}^{2} \wedge \hat{\omega}^{1}+\hat{\alpha}^{1} \wedge \hat{\omega}^{3}+\hat{\omega}^{6} \wedge \hat{\omega}^{7}+\epsilon \hat{\omega}^{2} \wedge \hat{\omega}^{5}+\hat{\omega}^{3} \wedge\left(\frac{\epsilon}{2 m} \hat{\omega}^{5}+\frac{3 m}{2} \hat{\omega}^{7}\right),
\end{aligned}
$$




$$
\begin{aligned}
& d \hat{\omega}^{4}=\hat{\alpha}^{1} \wedge \hat{\omega}^{4}+\epsilon \hat{\omega}^{5} \wedge \hat{\omega}^{6}-\hat{\omega}^{4} \wedge\left(\frac{3 \epsilon}{2 m} \hat{\omega}^{5}+\frac{m}{2} \hat{\omega}^{7}\right), \\
& d \hat{\omega}^{5}=\epsilon m \hat{\alpha}^{2} \wedge \hat{\omega}^{4}+m \hat{\omega}^{5} \wedge \hat{\omega}^{7}, \\
& d \hat{\omega}^{6}=\hat{\alpha}^{1} \wedge \hat{\omega}^{6}-\hat{\omega}^{4} \wedge \hat{\omega}^{7}-\hat{\omega}^{6} \wedge\left(\frac{\epsilon}{2 m} \hat{\omega}^{5}+\frac{3 m}{2} \hat{\omega}^{7}\right), \\
& d \hat{\omega}^{7}=\hat{\alpha}^{2} \wedge \hat{\omega}^{6}-\frac{\epsilon}{m} \hat{\omega}^{5} \wedge \hat{\omega}^{7}, \\
& d \hat{\alpha}^{1}=\frac{1}{2} \hat{\alpha}^{2} \wedge\left(\hat{\omega}^{4}+m \hat{\omega}^{6}\right), \\
& d \hat{\alpha}^{2}=\hat{\alpha}^{2} \wedge\left(\hat{\alpha}^{1}+\frac{3}{2}\left(\frac{\epsilon}{m} \hat{\omega}^{5}+m \hat{\omega}^{7}\right)\right) .
\end{aligned}
$$

\subsection{Case 2: $\Delta_{2}=0, B$ constant}

This branch contains two families of equations with 8-dimensional symmetry. All coefficients in both sets of structure equations are constants.

\subsubsection{Case 2a: $m=n=0$}

$$
\begin{aligned}
& d \hat{\omega}^{1}=2 \hat{\alpha}^{1} \wedge \hat{\omega}^{1}+\hat{\omega}^{3} \wedge \hat{\omega}^{6}+\hat{\omega}^{2} \wedge \hat{\omega}^{4}, \\
& d \hat{\omega}^{2}=\hat{\alpha}^{1} \wedge \hat{\omega}^{2}+\hat{\omega}^{4} \wedge \hat{\omega}^{5}+\hat{\omega}^{3} \wedge \hat{\omega}^{7}, \\
& d \hat{\omega}^{3}=\hat{\alpha}^{1} \wedge \hat{\omega}^{3}+\hat{\omega}^{6} \wedge \hat{\omega}^{7}+\epsilon \hat{\omega}^{2} \wedge \hat{\omega}^{5}, \\
& d \hat{\omega}^{4}=\hat{\alpha}^{1} \wedge \hat{\omega}^{4}+\epsilon \hat{\omega}^{5} \wedge \hat{\omega}^{6}+b \hat{\omega}^{2} \wedge \hat{\omega}^{5}+\epsilon \hat{\omega}^{2} \wedge \hat{\omega}^{7}, \\
& d \hat{\omega}^{5}=0, \\
& d \hat{\omega}^{6}=\hat{\alpha}^{1} \wedge \hat{\omega}^{6}-\hat{\omega}^{4} \wedge \hat{\omega}^{7}+\epsilon \hat{\omega}^{3} \wedge \hat{\omega}^{5}+\epsilon b \hat{\omega}^{3} \wedge \hat{\omega}^{7}, \\
& d \hat{\omega}^{7}=0, \\
& d \hat{\alpha}^{1}=0 .
\end{aligned}
$$

\subsubsection{Case 2b: $n=\epsilon_{1} m \neq 0($ and $\epsilon=1)$}

$$
\begin{aligned}
& d \hat{\omega}^{1}=2 \hat{\alpha}^{1} \wedge \hat{\omega}^{1}+\hat{\omega}^{3} \wedge \hat{\omega}^{6}+\hat{\omega}^{2} \wedge \hat{\omega}^{4}, \\
& d \hat{\omega}^{2}=\hat{\alpha}^{1} \wedge \hat{\omega}^{2}+\hat{\omega}^{4} \wedge \hat{\omega}^{5}+\hat{\omega}^{3} \wedge \hat{\omega}^{7}-\hat{\omega}^{2} \wedge\left(\frac{3 \epsilon_{1} m}{2} \hat{\omega}^{5}-\frac{m}{2} \hat{\omega}^{7}\right), \\
& d \hat{\omega}^{3}=\hat{\alpha}^{1} \wedge \hat{\omega}^{3}+\hat{\omega}^{6} \wedge \hat{\omega}^{7}+\epsilon \hat{\omega}^{2} \wedge \hat{\omega}^{5}-\hat{\omega}^{3} \wedge\left(\frac{\epsilon_{1} m}{2} \hat{\omega}^{5}-\frac{3 m}{2} \hat{\omega}^{7}\right), \\
& d \hat{\omega}^{4}=\hat{\alpha}^{1} \wedge \hat{\omega}^{4}+\epsilon \hat{\omega}^{5} \wedge \hat{\omega}^{6}+\left(m^{2}+\epsilon_{1}\right) \hat{\omega}^{2} \wedge\left(-2 \hat{\omega}^{5}+\epsilon_{1} \hat{\omega}^{7}\right)+\hat{\omega}^{4} \wedge\left(\frac{3 \epsilon_{1} m}{2} \hat{\omega}^{5}-\frac{m}{2} \hat{\omega}^{7}\right), \\
& d \hat{\omega}^{5}=m \hat{\omega}^{5} \wedge \hat{\omega}^{7}, \\
& d \hat{\omega}^{6}=\hat{\alpha}^{1} \wedge \hat{\omega}^{6}-\hat{\omega}^{4} \wedge \hat{\omega}^{7}+\left(m^{2}+\epsilon_{1}\right) \hat{\omega}^{3} \wedge\left(\epsilon_{1} \hat{\omega}^{5}-2 \hat{\omega}^{7}\right)+\hat{\omega}^{6} \wedge\left(\frac{\epsilon_{1} m}{2} \hat{\omega}^{5}-\frac{3 m}{2} \hat{\omega}^{7}\right), \\
& d \hat{\omega}^{7}=\epsilon_{1} m \hat{\omega}^{5} \wedge \hat{\omega}^{7}, \\
& d \hat{\alpha}^{1}=0 .
\end{aligned}
$$

\subsection{Case 3: $B$ nonconstant}

This branch contains two families of equations with 7-dimensional symmetry. Note the case $\Delta_{1}=\Delta_{2}=0, \epsilon=m=-n=1$ is contained in both families. 


\subsubsection{Case 3a: $\Delta_{1}=\mathbf{0}, \boldsymbol{B}$ nonconstant}

$$
\begin{aligned}
& d \hat{\omega}^{1}=2 \hat{\alpha}^{1} \wedge \hat{\omega}^{1}+\hat{\omega}^{2} \wedge \hat{\omega}^{4}+\hat{\omega}^{3} \wedge \hat{\omega}^{6}, \\
& d \hat{\omega}^{2}=\hat{\alpha}^{1} \wedge \hat{\omega}^{2}+\hat{\omega}^{4} \wedge \hat{\omega}^{5}+\hat{\omega}^{3} \wedge \hat{\omega}^{7}+\hat{\omega}^{2} \wedge\left(\frac{3 \epsilon}{2 m} \hat{\omega}^{5}+\frac{m}{2} \hat{\omega}^{7}\right), \\
& d \hat{\omega}^{3}=\hat{\alpha}^{1} \wedge \hat{\omega}^{3}+\hat{\omega}^{6} \wedge \hat{\omega}^{7}+\epsilon \hat{\omega}^{2} \wedge \hat{\omega}^{5}+\hat{\omega}^{3} \wedge\left(\frac{\epsilon}{2 m} \hat{\omega}^{5}+\frac{3 m}{2} \hat{\omega}^{7}\right), \\
& d \hat{\omega}^{4}=\hat{\alpha}^{1} \wedge \hat{\omega}^{4}+\epsilon \hat{\omega}^{5} \wedge \hat{\omega}^{6}+b e^{2 h} \hat{\omega}^{2} \wedge \hat{\omega}^{5}-\hat{\omega}^{4} \wedge\left(\frac{3 \epsilon}{2 m} \hat{\omega}^{5}+\frac{m}{2} \hat{\omega}^{7}\right), \\
& d \hat{\omega}^{5}=m \hat{\omega}^{5} \wedge \hat{\omega}^{7}, \\
& d \hat{\omega}^{6}=\hat{\alpha}^{1} \wedge \hat{\omega}^{6}-\hat{\omega}^{4} \wedge \hat{\omega}^{7}+\epsilon b e^{2 h} \hat{\omega}^{3} \wedge \hat{\omega}^{7}-\hat{\omega}^{6} \wedge\left(\frac{\epsilon}{2 m} \hat{\omega}^{5}+\frac{3 m}{2} \hat{\omega}^{7}\right), \\
& d \hat{\omega}^{7}=-\frac{\epsilon}{m} \hat{\omega}^{5} \wedge \hat{\omega}^{7}, \\
& d \hat{\alpha}^{1}=0 .
\end{aligned}
$$

On the level set $\{h=0\}$ : In this case, $\hat{\omega}^{7}=-\frac{\epsilon}{m^{2}} \hat{\omega}^{5}$.

$$
\begin{aligned}
& d \hat{\omega}^{1}=2 \hat{\alpha}^{1} \wedge \hat{\omega}^{1}+\hat{\omega}^{2} \wedge \hat{\omega}^{4}+\hat{\omega}^{3} \wedge \hat{\omega}^{6}, \\
& d \hat{\omega}^{2}=\hat{\alpha}^{1} \wedge \hat{\omega}^{2}+\left(\frac{\epsilon}{m} \hat{\omega}^{2}-\frac{\epsilon}{m^{2}} \hat{\omega}^{3}+\hat{\omega}^{4}\right) \wedge \hat{\omega}^{5}, \\
& d \hat{\omega}^{3}=\hat{\alpha}^{1} \wedge \hat{\omega}^{3}+\left(\epsilon \hat{\omega}^{2}-\frac{\epsilon}{m} \hat{\omega}^{3}-\frac{\epsilon}{m^{2}} \hat{\omega}^{6}\right) \wedge \hat{\omega}^{5}, \\
& d \hat{\omega}^{4}=\hat{\alpha}^{1} \wedge \hat{\omega}^{4}+\left(b \hat{\omega}^{2}-\frac{\epsilon}{m} \hat{\omega}^{4}-\epsilon \hat{\omega}^{6}\right) \wedge \hat{\omega}^{5}, \\
& d \hat{\omega}^{5}=0, \\
& d \hat{\omega}^{6}=\hat{\alpha}^{1} \wedge \hat{\omega}^{6}-\frac{1}{m^{2}}\left(b \hat{\omega}^{3}-\epsilon \hat{\omega}^{4}-\epsilon m \hat{\omega}^{6}\right) \wedge \hat{\omega}^{5}, \\
& d \hat{\alpha}^{1}=0 .
\end{aligned}
$$

\subsubsection{Case 3b: $\Delta_{2}=\mathbf{0}, B$ nonconstant}

$$
\begin{aligned}
d \hat{\omega}^{1}= & 2 \hat{\alpha}^{1} \wedge \hat{\omega}^{1}+\hat{\omega}^{3} \wedge \hat{\omega}^{6}+\hat{\omega}^{2} \wedge \hat{\omega}^{4}, \\
d \hat{\omega}^{2}= & \hat{\alpha}^{1} \wedge \hat{\omega}^{2}+\hat{\omega}^{4} \wedge \hat{\omega}^{5}+\hat{\omega}^{3} \wedge \hat{\omega}^{7}-\hat{\omega}^{2} \wedge\left(\frac{3 \epsilon_{1} m}{2} \hat{\omega}^{5}-\frac{m}{2} \hat{\omega}^{7}\right), \\
d \hat{\omega}^{3}= & \hat{\alpha}^{1} \wedge \hat{\omega}^{3}+\hat{\omega}^{6} \wedge \hat{\omega}^{7}+\hat{\omega}^{2} \wedge \hat{\omega}^{5}-\hat{\omega}^{3} \wedge\left(\frac{\epsilon_{1} m}{2} \hat{\omega}^{5}-\frac{3 m}{2} \hat{\omega}^{7}\right), \\
d \hat{\omega}^{4}= & \hat{\alpha}^{1} \wedge \hat{\omega}^{4}+\hat{\omega}^{5} \wedge \hat{\omega}^{6}+\left(-2\left(m^{2}+\epsilon_{1}\right)+b e^{2 h}\right) \hat{\omega}^{2} \wedge \hat{\omega}^{5}+\left(\epsilon_{1} m^{2}+1\right) \hat{\omega}^{2} \wedge \hat{\omega}^{7} \\
& +\hat{\omega}^{4} \wedge\left(\frac{3 \epsilon_{1} m}{2} \hat{\omega}^{5}-\frac{m}{2} \hat{\omega}^{7}\right), \\
d \hat{\omega}^{5}= & m \hat{\omega}^{5} \wedge \hat{\omega}^{7}, \\
d \hat{\omega}^{6}= & \hat{\alpha}^{1} \wedge \hat{\omega}^{6}-\hat{\omega}^{4} \wedge \hat{\omega}^{7}+\left(\epsilon_{1} m^{2}+1\right) \hat{\omega}^{3} \wedge \hat{\omega}^{5}+\left(-2\left(m^{2}+\epsilon_{1}\right)+b e^{2 h}\right) \hat{\omega}^{3} \wedge \hat{\omega}^{7} \\
& +\hat{\omega}^{6} \wedge\left(\frac{\epsilon_{1} m}{2} \hat{\omega}^{5}-\frac{3 m}{2} \hat{\omega}^{7}\right), \\
d \hat{\omega}^{7}= & \epsilon_{1} m \hat{\omega}^{5} \wedge \hat{\omega}^{7}, \\
d \hat{\alpha}^{1}= & 0 .
\end{aligned}
$$

On the level set $\{h=0\}$ : In this case, $\hat{\omega}^{7}=\epsilon_{1} \hat{\omega}^{5}$.

$$
d \hat{\omega}^{1}=2 \hat{\alpha}^{1} \wedge \hat{\omega}^{1}+\hat{\omega}^{3} \wedge \hat{\omega}^{6}+\hat{\omega}^{2} \wedge \hat{\omega}^{4},
$$




$$
\begin{aligned}
& d \hat{\omega}^{2}=\hat{\alpha}^{1} \wedge \hat{\omega}^{2}+\left(\hat{\omega}^{4}+\epsilon_{1} \hat{\omega}^{3}-\epsilon_{1} m \hat{\omega}^{2}\right) \wedge \hat{\omega}^{5}, \\
& d \hat{\omega}^{3}=\hat{\alpha}^{1} \wedge \hat{\omega}^{3}+\epsilon_{1}\left(\hat{\omega}^{6}+\epsilon_{1} \hat{\omega}^{2}+m \hat{\omega}^{3}\right) \wedge \hat{\omega}^{5}, \\
& d \hat{\omega}^{4}=\hat{\alpha}^{1} \wedge \hat{\omega}^{4}+\left(-\hat{\omega}^{6}+\left(-\left(m^{2}+\epsilon_{1}\right)+b\right) \hat{\omega}^{2}+\epsilon_{1} m \hat{\omega}^{4}\right) \wedge \hat{\omega}^{5}, \\
& d \hat{\omega}^{5}=0, \\
& d \hat{\omega}^{6}=\hat{\alpha}^{1} \wedge \hat{\omega}^{6}+\epsilon_{1}\left(-\hat{\omega}^{4}+\left(-\left(m^{2}+\epsilon_{1}\right)+b\right) \hat{\omega}^{3}-m \hat{\omega}^{6}\right) \wedge \hat{\omega}^{5}, \\
& d \hat{\alpha}^{1}=0 .
\end{aligned}
$$

\section{The maximally symmetric case}

\subsection{A coframing in local coordinates}

For the remainder of the paper we focus on the maximally symmetric generic hyperbolic structures. In Appendix E, we outline how Vranceanu arrived at an explicit coframe $\left\{\omega^{i}\right\}_{i=1}^{7}$ on $\Sigma_{7}$ given in local coordinates which satisfies the structure equations (6.5). In local coordinates $(x, y, z, p, q, u, v)$ on $\Sigma_{7}$, the coframe is given by

$$
\begin{aligned}
\omega^{1}= & d z-p d x-q d y, \\
\omega^{2}= & \left(\frac{\epsilon m^{2}}{6}-\frac{m \alpha v^{3}}{3 u^{3}}+\frac{\alpha v^{2}}{2 u^{2}}\right) \omega^{6}+\left(-\frac{\epsilon m}{3}-\frac{\alpha v^{3}}{3 u^{3}}\right) \omega^{4}-u^{-3 / 2}(d p+v d q), \\
\omega^{3}= & \left(\frac{\epsilon m^{2}}{6}-\frac{m \alpha v^{3}}{3 u^{3}}+\frac{\alpha v^{2}}{2 u^{2}}\right) \omega^{4}+\left(-\frac{\epsilon m^{3}}{3}-\frac{m^{2} \alpha v^{3}}{3 u^{3}}+\frac{m \alpha v^{2}}{u^{2}}-\frac{\alpha v}{u}\right) \omega^{6} \\
& -m u^{-3 / 2}(d p+v d q)+u^{-1 / 2} d q, \\
\omega^{4}= & u^{3 / 2} d x+m \sqrt{u}(d y-v d x), \quad \omega^{5}=\frac{\epsilon m(d u-m d v)}{u}, \\
\omega^{6}= & -\sqrt{u}(d y-v d x), \quad \omega^{7}=\frac{d v}{u},
\end{aligned}
$$

which is valid on the open set $u>0$, and where $\alpha=1-\epsilon m^{4}$. The coordinates $(x, y, z, p, q)$ are identified with the corresponding coordinates on $J^{1}\left(\mathbb{R}^{2}, \mathbb{R}\right)$.

Note that $\alpha$ in the case $\Delta_{1}=0$ is a relative contact invariant since $\alpha=-\epsilon m^{2}\left(m^{2}-\frac{\epsilon}{m^{2}}\right)=$ $-\epsilon m^{2} \Delta_{2}, m \neq 0$, and $\Delta_{2}$ is relative contact invariant. Since the contact-inequivalent structures are parametrized by $(\epsilon, m) \in\{ \pm 1\} \times(0,1]$, then $\alpha \in[0,1) \cup(1,2]$.

\subsection{Normal forms}

Let us determine how the coordinates $(u, v)$ on $\Sigma_{7}$ are related to the standard 2-jet coordinates $(x, y, z, p, q, r, s, t) \in J^{2}\left(\mathbb{R}^{2}, \mathbb{R}\right)$. Let $\chi: \mathbb{R}^{2} \rightarrow \Sigma_{7}$ be any integral manifold of $I_{F}$ with independence condition $\chi^{*}(d x \wedge d y) \neq 0$. Without loss of generality, we identify the coordinates $(x, y)$ on $\mathbb{R}^{2}$ with the $(x, y)$ coordinates on $\Sigma_{7}$. The composition $i_{F} \circ \chi$ is then an integral manifold of the contact system $\mathcal{C}^{(2)}$ and on $\mathbb{R}^{2}$ we can write

$$
d p=r d x+s d y, \quad d q=s d x+t d y,
$$

where for convenience $p$ is identified with $\left(i_{F} \circ \chi\right)^{*} p$, and similarly for the coordinates $q, r, s, t$. Substituting (7.2) into the conditions $0=\chi^{*} \omega^{2}=\chi^{*} \omega^{3}$, and extracting the coefficients of $d x$ and $d y$, we obtain the relations

$$
\begin{aligned}
& 0=6 v s+6 r+2 \epsilon m u^{3}-3 \epsilon m^{2} u^{2} v-\alpha v^{3}, \\
& 0=2 v t+2 s+\epsilon m^{2} u^{2}+\alpha v^{2},
\end{aligned}
$$




$$
\begin{aligned}
& 0=-6 s u+m\left(6 s v+6 r-\alpha v^{3}\right)+3 v \epsilon m^{3} u^{2}+3 \alpha v^{2} u-\epsilon m^{2} u^{3}, \\
& 0=-2 t u+m\left(2 t v+2 s+\alpha v^{2}\right)-\epsilon m^{3} u^{2}-2 \alpha v u
\end{aligned}
$$

or equivalently, using the coordinate $w=u-m v$ instead of $u$, we have

$$
r=-\frac{1}{3}\left(\epsilon m w^{3}+v^{3}\right), \quad s=-\frac{1}{2}\left(\epsilon m^{2} w^{2}-v^{2}\right), \quad t=-\left(\epsilon m^{3} w+v\right) .
$$

Thus, our PDE is of the form

$$
F(r, s, t)=0,
$$

and we have a nondegenerate parametrization $i_{F}: \Sigma_{7} \rightarrow J^{2}\left(\mathbb{R}^{2}, \mathbb{R}\right)($ for $u=w+m v>0$ ).

Consider the case $\alpha=0$, i.e. $(\epsilon, m)=(1,1)$. In this case, it is straightforward to eliminate both parameters $w, v$ and obtain the equation

$$
r t-s^{2}-\frac{t^{4}}{12}=0
$$

Now consider the general case $\alpha \neq 0$. Let us write $u=-\frac{1}{\tilde{u}}, v=\tilde{v}$ and rewrite (7.3) as

$$
\begin{aligned}
& \tilde{u} t=\epsilon m^{3}-\alpha \tilde{u} \tilde{v} \\
& \tilde{u}^{2} s=-\frac{1}{2} \epsilon m^{2}-\epsilon m^{3} \tilde{u} \tilde{v}+\frac{1}{2} \alpha(\tilde{u} \tilde{v})^{2}=-\frac{\epsilon m^{2}}{2 \alpha}+\frac{\tilde{u}^{2} t^{2}}{2 \alpha}, \\
& \tilde{u}^{3} r=\frac{1}{3} \epsilon m+\epsilon m^{2} \tilde{u} \tilde{v}+\epsilon m^{3}(\tilde{u} \tilde{v})^{2}-\frac{1}{3} \alpha(\tilde{u} \tilde{v})^{3}=\frac{m\left(\epsilon+m^{4}\right)}{3 \alpha^{2}}-\frac{\epsilon m^{2} \tilde{u} t}{\alpha^{2}}+\frac{\tilde{u}^{3} t^{3}}{3 \alpha^{2}},
\end{aligned}
$$

and so using $\nu=\left(\epsilon m^{3}-\alpha \tilde{u} \tilde{v}\right)^{-1}$ as a new parameter, we arrive at

$$
2 \alpha s-t^{2}=-\epsilon m^{2} \nu^{2} t^{2}, \quad 3 \alpha^{2} r=m\left(\epsilon+m^{4}\right) \nu^{3} t^{3}-3 \epsilon m^{2} \nu^{2} t^{3}+t^{3} .
$$

Eliminating the parameter $\nu$, we obtain

$$
\left(\epsilon+m^{4}\right)^{2}\left(2 \alpha s-t^{2}\right)^{3}+\epsilon m^{4}\left(3 \alpha^{2} r-6 \alpha s t+2 t^{3}\right)^{2}=0 .
$$

Finally, use the scaling $\bar{x}=\frac{1}{\alpha} x$, which induces

$$
(\bar{r}, \bar{s}, \bar{t})=\left(\alpha^{2} r, \alpha s, t\right)
$$

to eliminate $\alpha$ from (7.5). Dropping bars, and letting $a=m^{4}$ we obtain

$$
(\epsilon+a)^{2}\left(2 s-t^{2}\right)^{3}+\epsilon a\left(3 r-6 s t+2 t^{3}\right)^{2}=0 .
$$

Note that in the case $\epsilon=1$ considered by Vranceanu, the st term has a missing factor of 2 .

Theorem 7.1. The contact-equivalence classes of maximally symmetric generic hyperbolic PDE are parametrized by $(\epsilon, a) \in\{ \pm 1\} \times(0,1]$. Normal forms from each equivalence class are given by (7.4) in the case $(\epsilon, a)=(1,1)$ and $(7.6)$ otherwise.

Remark 7.1. Letting $\epsilon=a=1$ in (7.6), we have $F=4\left(2 s-t^{2}\right)^{3}+\left(3 r-6 s t+2 t^{3}\right)^{2}=0$, and

$$
\Delta=F_{r} F_{t}-\frac{1}{4} F_{s}^{2}=-36\left(2 s-t^{2}\right) F .
$$

On the equation manifold (and hence on $\Sigma_{7}$ ), we have $\Delta=0$ and consequently, this limiting equation is parabolic. 


\subsection{Nine-dimensional symmetry algebras}

The calculations leading to (7.3) are quite long and consequently to confirm the validity of (7.3) (and in turn, Theorem 7.1), it is useful to describe the nine-dimensional (contact) symmetry algebra explicitly for the normal forms given in the previous section. Calculating the symmetry algebra is a nontrivial task however - the standard Lie method of calculating symmetries (by working in $J^{2}\left(\mathbb{R}^{2}, \mathbb{R}\right)$ on the equation locus) is highly impractical owing to the complexity of the equations. In Appendix F, we describe how the symmetry algebra was found by an alternative method. In order to give a unified description of the symmetry algebras, we work with the normal forms (7.4) and (7.5) as these arise from the parametrization (7.3).

Proposition 7.1. Any equation of the form $F(r, s, t)=0$ admits the symmetries

$$
\begin{aligned}
& X_{1}=\frac{\partial}{\partial x}, \quad X_{2}=\frac{\partial}{\partial y}, \quad X_{3}=\frac{\partial}{\partial z}, \quad X_{4}=x \frac{\partial}{\partial z}, \quad X_{5}=y \frac{\partial}{\partial z}, \\
& X_{6}=x \frac{\partial}{\partial x}+y \frac{\partial}{\partial y}+2 z \frac{\partial}{\partial z} .
\end{aligned}
$$

The equations (7.4) and (7.5) have the following additional symmetries:

$$
X_{7}=y \frac{\partial}{\partial y}+3 z \frac{\partial}{\partial z}, \quad X_{8}=x \frac{\partial}{\partial y}-\frac{\alpha}{2} y^{2} \frac{\partial}{\partial z}, \quad X_{9}=x^{2} \frac{\partial}{\partial x}+x y \frac{\partial}{\partial y}+\left(x z-\frac{\alpha}{6} y^{3}\right) \frac{\partial}{\partial z} .
$$

In particular, all of these symmetries are projectable point symmetries.

(Recall that a point symmetry here is a vector field on $J^{0}\left(\mathbb{R}^{2}, \mathbb{R}\right)$. A point symmetry is projectable if it projects to a vector field on the base $\mathbb{R}^{2}$.)

The normalization of (7.5) to (7.6) is carried out by letting $\bar{x}=\frac{1}{\alpha} x$ from which we get:

Corollary 7.1. The generic hyperbolic equation (7.6) has symmetry generators $X_{1}, \ldots, X_{6}$ as in Proposition 7.1 as well as

$$
X_{7}=y \frac{\partial}{\partial y}+3 z \frac{\partial}{\partial z}, \quad X_{8}=x \frac{\partial}{\partial y}-\frac{1}{2} y^{2} \frac{\partial}{\partial z}, \quad X_{9}=x^{2} \frac{\partial}{\partial x}+x y \frac{\partial}{\partial y}+\left(x z-\frac{1}{6} y^{3}\right) \frac{\partial}{\partial z} .
$$

We will denote the corresponding abstract Lie algebras as $\mathfrak{g}_{\alpha}$ and express their commutator relations in a canonical basis. Let

$$
\left(e_{1}, e_{2}, e_{3}, e_{4}, e_{5}, e_{6}, e_{7}, e_{8}, e_{9}\right)=\left(X_{2}, X_{3}, X_{4}, X_{5},-X_{8}, X_{7}, X_{1},-2 X_{6}+X_{7},-X_{9}\right) \text {. }
$$

The commutator relations in this basis are

\begin{tabular}{c|cccccc|ccc} 
& $e_{1}$ & $e_{2}$ & $e_{3}$ & $e_{4}$ & $e_{5}$ & $e_{6}$ & $e_{7}$ & $e_{8}$ & $e_{9}$ \\
\hline$e_{1}$ & $\cdot$ & $\cdot$ & $\cdot$ & $e_{2}$ & $\alpha e_{4}$ & $e_{1}$ & $\cdot$ & $-e_{1}$ & $e_{5}$ \\
$e_{2}$ & & $\cdot$ & $\cdot$ & $\cdot$ & $\cdot$ & $3 e_{2}$ & $\cdot$ & $-e_{2}$ & $-e_{3}$ \\
$e_{3}$ & & & $\cdot$ & $\cdot$ & $\cdot$ & $3 e_{3}$ & $-e_{2}$ & $e_{3}$ & $\cdot$ \\
$e_{4}$ & & & & $\cdot$ & $e_{3}$ & $2 e_{4}$ & $\cdot$ & $\cdot$ & $\cdot$ \\
$e_{5}$ & & & & & $\cdot$ & $e_{5}$ & $e_{1}$ & $e_{5}$ & $\cdot$ \\
$e_{6}$ & & & & & & $\cdot$ & $\cdot$ & $\cdot$ & $\cdot$ \\
\hline$e_{7}$ & & & & & & & $\cdot$ & $-2 e_{7}$ & $e_{8}$ \\
$e_{8}$ & & & & & & & & $\cdot$ & $-2 e_{9}$ \\
$e_{9}$ & & & & & & & & & $\cdot$
\end{tabular}


In the case $\alpha \neq 0$, redefining

$$
\left(\bar{e}_{2}, \bar{e}_{3}, \bar{e}_{4}\right)=\left(\alpha e_{2}, \alpha e_{3}, \alpha e_{4}\right)
$$

and dropping the bars, we have the same commutator relations as above except $\alpha$ has been normalized to 1 . Thus, in the case $\alpha \neq 0$, all symmetry algebras are isomorphic. (This is also obvious from the fact that the symmetry generators in Corollary 7.1 are independent of $\alpha$.)

Let $\mathfrak{g}_{1}$ denote the abstract symmetry algebra in the case $\alpha \neq 0$, although this is a slight abuse of notation since $\alpha \in(0,1) \cup(1,2]$ in this case. We calculate for $\mathfrak{g}=\mathfrak{g}_{\delta}(\delta=0,1)$,

$$
\begin{array}{ll}
\text { Killing form: } & \kappa=\operatorname{diag}\left(0,0,0,0,0,24,\left(\begin{array}{ccc}
0 & 0 & 6 \\
0 & 12 & 0 \\
6 & 0 & 0
\end{array}\right)\right), \\
\text { derived subalgebra: } & \mathfrak{g}^{(1)}=\left\langle e_{1}, e_{2}, e_{3}, e_{4}, e_{5}, e_{7}, e_{8}, e_{9}\right\rangle, \\
\text { radical: } & \mathfrak{r}=\left(\mathfrak{g}^{(1)}\right)^{\perp \kappa}=\left\langle e_{1}, e_{2}, e_{3}, e_{4}, e_{5}, e_{6}\right\rangle, \\
\text { (semi-simple) Levi factor: } & \mathfrak{g}_{s s}=\left\langle e_{7}, e_{8}, e_{9}\right\rangle \cong \mathfrak{s} \mathfrak{l}(2, \mathbb{R}), \\
\text { Levi decomposition: } & \mathfrak{g}=\mathfrak{r} \rtimes \mathfrak{g}_{s s}, \\
\text { nilradical : } & \mathfrak{n}=\left\langle e_{1}, e_{2}, e_{3}, e_{4}, e_{5}\right\rangle, \\
\text { derived series of } \mathfrak{r}: & \mathfrak{r}^{(1)}=\mathfrak{n}, \quad \mathfrak{r}^{(2)}=\left\langle e_{2}, e_{3}, \delta e_{4}\right\rangle, \quad \mathfrak{r}^{(\infty)}=\mathfrak{r}^{(3)}=0, \\
\text { lower central series of } \mathfrak{r}: & \mathfrak{r}^{\infty}=\mathfrak{r}^{1}=\mathfrak{n} .
\end{array}
$$

An isomorphism between two Lie algebras must restrict to an isomorphism of their radicals and the corresponding derived flags of the radicals. Since $\mathfrak{r}^{(2)}$ is two-dimensional for $\mathfrak{g}_{0}$ and three-dimensional for $\mathfrak{g}_{1}$, then we must have $\mathfrak{g}_{0} \neq \mathfrak{g}_{1}$.

Theorem 7.2. The contact symmetry algebra of any maximally symmetric generic hyperbolic $P D E$ is:

1) nine-dimensional,

2) contact-equivalent to a (projectable) point symmetry algebra.

Moreover, there are exactly two isomorphism classes of Lie algebras (represented by $\mathfrak{g}_{0}$ and $\mathfrak{g}_{1}$ ) that arise as such symmetry algebras.

We remark that Mubarakzjanov has classified all five-dimensional real solvable Lie algebras (labelled by $g_{5, *}$ ) [20] and all six-dimensional non-nilpotent real solvable Lie algebras (labelled by $\left.g_{6, *}\right)$ [21]. The nilradicals of $\mathfrak{g}_{0}$ and $\mathfrak{g}_{1}$ can be identified in the former classification as:

$$
\begin{array}{ll}
\mathfrak{n}_{0} \cong g_{5,1}: & \left(\bar{e}_{1}, \bar{e}_{2}, \bar{e}_{3}, \bar{e}_{4}, \bar{e}_{5}\right)=\left(e_{2},-e_{3}, e_{1}, e_{5}, e_{4}\right), \\
\mathfrak{n}_{1} \cong g_{5,3}: & \left(\bar{e}_{1}, \bar{e}_{2}, \bar{e}_{3}, \bar{e}_{4}, \bar{e}_{5}\right)=\left(e_{3}, e_{4},-e_{2}, e_{1}, e_{5}\right) .
\end{array}
$$

The radicals of $\mathfrak{g}_{0}$ and $\mathfrak{g}_{1}$ can be identified in the latter classification as

$$
\begin{aligned}
& \mathfrak{r}_{0} \cong g_{6,54}: \quad\left(\bar{e}_{1}, \bar{e}_{2}, \bar{e}_{3}, \bar{e}_{4}, \bar{e}_{5}, \bar{e}_{6}\right)=\left(e_{2},-e_{3}, e_{1}, e_{5}, e_{4}, \frac{1}{3} e_{6}\right), \quad \text { param.: }(\lambda, \gamma)=\left(1, \frac{2}{3}\right), \\
& \mathfrak{r}_{1} \cong g_{6,76}: \quad\left(\bar{e}_{1}, \bar{e}_{2}, \bar{e}_{3}, \bar{e}_{4}, \bar{e}_{5}, \bar{e}_{6}\right)=\left(e_{3}, e_{4},-e_{2}, e_{1}, e_{5}, \frac{1}{3} e_{6}\right), \quad \text { param.: } h=1 .
\end{aligned}
$$

Let us be more explicit about the direct verification of Proposition 7.1 from the point of view of external symmetries, internal symmetries, and symmetries of the lifted coframe on $\Sigma_{7} \times H$. 


\subsubsection{External symmetries}

Given any vector field $X$ on $J^{0}\left(\mathbb{R}^{2}, \mathbb{R}\right)$, there is a corresponding prolonged vector field $X^{(2)}$ on $J^{2}\left(\mathbb{R}^{2}, \mathbb{R}\right)$. This prolongation is uniquely determined by the condition that $\mathcal{L}_{X^{(2)}} \mathcal{C}^{(2)} \subset \mathcal{C}^{(2)}$, where $\mathcal{C}^{(2)}$ is the contact system on $J^{2}\left(\mathbb{R}^{2}, \mathbb{R}\right)$. See (F.5) for the standard prolongation formula. For the vector fields in Proposition 7.1, we have

$$
\begin{aligned}
& X_{1}^{(2)}=X_{1}, \quad X_{2}^{(2)}=X_{2}, \quad X_{3}^{(2)}=X_{3}, \\
& X_{4}^{(2)}=X_{4}+\frac{\partial}{\partial p}, \quad X_{5}^{(2)}=X_{5}+\frac{\partial}{\partial q}, \quad X_{6}^{(2)}=X_{6}+p \frac{\partial}{\partial p}+q \frac{\partial}{\partial q}, \\
& X_{7}^{(2)}=X_{7}+3 p \frac{\partial}{\partial p}+2 q \frac{\partial}{\partial q}+3 r \frac{\partial}{\partial r}+2 s \frac{\partial}{\partial s}+t \frac{\partial}{\partial t}, \\
& X_{8}^{(2)}=X_{8}-q \frac{\partial}{\partial p}-\alpha y \frac{\partial}{\partial q}-2 s \frac{\partial}{\partial r}-t \frac{\partial}{\partial s}-\alpha \frac{\partial}{\partial t}, \\
& X_{9}^{(2)}=X_{9}+(z-x p-y q) \frac{\partial}{\partial p}-\frac{\alpha}{2} y^{2} \frac{\partial}{\partial q}-(3 x r+2 y s) \frac{\partial}{\partial r}-(2 x s+y t) \frac{\partial}{\partial s}-(x t+\alpha y) \frac{\partial}{\partial t} .
\end{aligned}
$$

For (7.4) or (7.5), we verify the external symmetry condition

$$
\mathcal{L}_{X_{i}(2)} F=0 \quad \text { whenever } \quad F=0 .
$$

Clearly this is satisfied by $X_{i}^{(2)}, i=1, \ldots, 6$ since they have no components in the $\frac{\partial}{\partial r}, \frac{\partial}{\partial s}, \frac{\partial}{\partial t}$ direction and since $F=F(r, s, t)$ for (7.4) and (7.5). For the remaining vector fields we have

$$
\begin{array}{llll}
(7.4): & \mathcal{L}_{X_{7}^{(2)}} F=4 F, & \mathcal{L}_{X_{8}^{(2)}} F=0, & \mathcal{L}_{X_{9}^{(2)}} F=-4 x F, \\
(7.5): & \mathcal{L}_{X_{7}^{(2)}} F=6 F, & \mathcal{L}_{X_{8}^{(2)}} F=0, & \mathcal{L}_{X_{9}^{(2)}} F=-6 x F,
\end{array}
$$

and so the external symmetry condition is satisfied.

\subsubsection{Internal symmetries}

The symmetry generators $X_{i}^{(2)}$ are all tangent to the equation manifold $F=0$, so they induce (via the parametrization $(7.3)$ ) corresponding vector fields $Z_{i}$ on $\Sigma_{7}$. Letting $X_{i}^{(1)}=\left(\pi_{1}^{2}\right)_{*} X_{i}^{(2)}$ denote the projection onto $J^{1}\left(\mathbb{R}^{2}, \mathbb{R}\right)$, and identifying the coordinates $(x, y, z, p, q)$ on $J^{1}\left(\mathbb{R}^{2}, \mathbb{R}\right)$ with corresponding coordinates on $\Sigma_{7}$, we have

$$
\begin{aligned}
Z_{i} & =X_{i}^{(1)}, \quad i=1, \ldots, 6, & Z_{7} & =X_{7}^{(1)}+w \frac{\partial}{\partial w}+v \frac{\partial}{\partial v}, \\
Z_{8} & =X_{8}^{(1)}+\frac{\partial}{\partial v}-m \frac{\partial}{\partial w}, & Z_{9} & =X_{9}^{(1)}-(m y+x w) \frac{\partial}{\partial w}+(y-x v) \frac{\partial}{\partial v},
\end{aligned}
$$

with $u=w+m v$. One can verify directly that these vector fields satisfy the internal symmetry condition

$$
\mathcal{L}_{Z_{i}} I_{F} \subset I_{F}
$$

where $I_{F}=\left\langle\omega^{1}, \omega^{2}, \omega^{3}\right\rangle$ is given by the explicit coframing (7.1).

\subsubsection{Symmetries of the lifted coframe on $\Sigma_{7} \times H^{\prime}$, where $H^{\prime}=H^{0} \rtimes\left\langle R^{2}\right\rangle$}

The lifted coframe $\hat{\boldsymbol{\omega}}=\left\{\hat{\omega}^{1}, \ldots, \hat{\omega}^{7}, \hat{\alpha}^{1}, \hat{\alpha}^{2}\right\}$ on $\Sigma_{7} \times H^{\prime}$ is parametrized by

$$
\hat{\omega}^{1}=a_{1}{ }^{2} \omega^{1}, \quad \hat{\omega}^{2}=a_{1} \omega^{2}+a_{1} a_{2} \omega^{1}, \quad \hat{\omega}^{3}=a_{1} \omega^{3}+m a_{1} a_{2} \omega^{1},
$$




$$
\begin{aligned}
& \hat{\omega}^{4}=a_{1} \omega^{4}, \quad \hat{\omega}^{5}=\omega^{5}+\epsilon m a_{2} \omega^{4}, \quad \hat{\omega}^{6}=a_{1} \omega^{6}, \quad \hat{\omega}^{7}=\omega^{7}+a_{2} \omega^{6}, \\
& \hat{\alpha}^{1}=\frac{d a_{1}}{a_{1}}+\frac{a_{2}}{2 a_{1}}\left(\hat{\omega}^{4}+m \hat{\omega}^{6}\right), \quad \hat{\alpha}^{2}=\frac{d a_{2}}{a_{1}}+\frac{3 a_{2}}{2 a_{1}}\left(\frac{\epsilon}{m} \hat{\omega}^{5}+m \hat{\omega}^{7}\right)-\frac{a_{2}{ }^{2}}{2 a_{1}{ }^{2}}\left(\hat{\omega}^{4}+m \hat{\omega}^{6}\right),
\end{aligned}
$$

and by construction $\hat{\omega}$ is an $\{e\}$-structure on $\Sigma_{7} \times H^{\prime}$, so that a symmetry is by definition a map $\Phi: \Sigma_{7} \times H^{\prime} \rightarrow \Sigma_{7} \times H^{\prime}$ such that

$$
\Phi^{*} \hat{\boldsymbol{\omega}}^{i}=\hat{\boldsymbol{\omega}}^{i}, \quad i=1, \ldots, 9,
$$

with infinitesimal analogue

$$
\mathcal{L}_{\hat{Z}} \hat{\omega}^{i}=0, \quad i=1, \ldots, 9 .
$$

Explicitly, these lifted vector fields are given by

$$
\begin{aligned}
& \hat{Z}_{i}=Z_{i}, \quad i=1, \ldots, 5, \quad \hat{Z}_{6}=Z_{6}-a_{1} \frac{\partial}{\partial a_{1}}-a_{2} \frac{\partial}{\partial a_{2}}, \quad \hat{Z}_{7}=Z_{7}-\frac{3 a_{1}}{2} \frac{\partial}{\partial a_{1}}-\frac{3 a_{2}}{2} \frac{\partial}{\partial a_{2}}, \\
& \hat{Z}_{8}=Z_{8}, \quad \hat{Z}_{9}=Z_{9}-\frac{x a_{1}}{2} \frac{\partial}{\partial a_{1}}+\left(\frac{1}{(w+m v)^{3 / 2}}-\frac{x a_{2}}{2}\right) \frac{\partial}{\partial a_{2}} .
\end{aligned}
$$

\subsection{Ampère contact transformations and $3 z_{x x}\left(z_{y y}\right)^{3}+1=0$}

After deriving the normal form

$$
r t-s^{2}-\frac{t^{4}}{12}=0
$$

which appeared in (7.4), Vranceanu remarks that if one makes an Ampère contact transformation, then (7.7) can be reduced to the simpler form

$$
r t^{3}+\frac{1}{12}=0
$$

The notion of an Ampère contact transformation is never defined in Vranceanu's paper and does not appear to be common terminology in the literature. This terminology is, however, referred to briefly in recent work by Stormark (see page 275 in [24]). Namely, Stormark defines it as the genuine (i.e. non-point) contact transformation $\Phi$ of $J^{1}\left(\mathbb{R}^{2}, \mathbb{R}\right)$ given by

$$
(\bar{x}, \bar{y}, \bar{z}, \bar{p}, \bar{q})=(p, y, z-p x,-x, q)
$$

which is clearly contact since

$$
d \bar{z}-\bar{p} d \bar{x}-\bar{q} d \bar{y}=d(z-p x)+x d p-q d y=d z-p d x-q d y .
$$

This is essentially akin to the Legendre transformation from Hamiltonian mechanics, but only acting with respect to the $x, z, p$ variables. For our purposes, we consider the corresponding Legendre-like transformation acting with respect to $y, z, q$ variables, namely

$$
(\bar{x}, \bar{y}, \bar{z}, \bar{p}, \bar{q})=(x, q, z-q y, p,-y) .
$$

The prolongation of this transformation to $J^{2}\left(\mathbb{R}^{2}, \mathbb{R}\right)$ satisfies

$$
\begin{aligned}
d \bar{p}-\bar{r} d \bar{x}-\bar{s} d \bar{y} & =d p-\bar{r} d x-\bar{s} d q \equiv r d x+s d y-\bar{r} d x-\bar{s}(s d x+t d y) & \bmod \mathcal{C}^{(2)} \\
& \equiv(r-s \bar{s}-\bar{r}) d x+(s-t \bar{s}) d y \quad \bmod \mathcal{C}^{(2)}, & \\
d \bar{q}-\bar{s} d \bar{x}-\bar{t} d \bar{y} & =-d y-\bar{s} d x-\bar{t} d q \equiv-d y-\bar{s} d x-\bar{t}(s d x+t d y) & \bmod \mathcal{C}^{(2)}
\end{aligned}
$$




$$
\equiv-(\bar{s}+s \bar{t}) d x-(1+t \bar{t}) d y \quad \bmod \mathcal{C}^{(2)},
$$

and hence

$$
(\bar{r}, \bar{s}, \bar{t})=\left(\frac{r t-s^{2}}{t}, \frac{s}{t},-\frac{1}{t}\right) .
$$

Consequently

$$
0=r t-s^{2}-\frac{t^{4}}{12}=-\frac{\bar{r}}{\bar{t}}-\frac{1}{12 \bar{t}^{4}}=-\frac{1}{\bar{t}^{4}}\left(\bar{r} \bar{t}^{3}+\frac{1}{12}\right) \quad \Rightarrow \quad \bar{r} \bar{t}^{3}+\frac{1}{12}=0 .
$$

By applying the subsequent scaling $x=\frac{1}{2} \bar{x}$ (and hence $(r, s, t)=(4 \bar{r}, 2 \bar{s}, \bar{t})$ ), we are led to the equation

$$
3 r t^{3}+1=0,
$$

which was investigated by Goursat [12] who recognized its Darboux integrability.

Since (7.9) is contact-equivalent to (7.7), it is clear that (7.9) is hyperbolic of generic type with $\Delta_{1}=\Delta_{2}=0$ and $\epsilon=a=1$. The standard Lie algorithm to calculate symmetries can be applied for this equation in a straightforward manner. Its contact symmetry algebra consists of (projectable) point symmetries $X_{1}, \ldots, X_{6}$ as in Proposition 7.1 as well as

$$
X_{7}=x y \frac{\partial}{\partial z}, \quad X_{8}=2 y \frac{\partial}{\partial y}+3 z \frac{\partial}{\partial z}, \quad X_{9}=x^{2} \frac{\partial}{\partial x}+x z \frac{\partial}{\partial z} .
$$

We note that the vector fields $X_{7}, X_{8}, X_{9}$ have prolongations

$$
\begin{aligned}
& X_{7}^{(2)}=X_{7}+y \frac{\partial}{\partial p}+x \frac{\partial}{\partial q}+\frac{\partial}{\partial s}, \\
& X_{8}^{(2)}=X_{8}+3 p \frac{\partial}{\partial p}+q \frac{\partial}{\partial q}+3 r \frac{\partial}{\partial r}+s \frac{\partial}{\partial s}-t \frac{\partial}{\partial t}, \\
& X_{9}^{(2)}=X_{9}+(z-x p) \frac{\partial}{\partial p}+x q \frac{\partial}{\partial q}-3 x r \frac{\partial}{\partial r}+(q-x s) \frac{\partial}{\partial s}+x t \frac{\partial}{\partial t} .
\end{aligned}
$$

\subsection{Darboux integrability}

Definition 7.1. For a hyperbolic PDE $F=0, I_{F}$ is said to be Darboux-integrable (at level two) if each of $C\left(I_{F}, d M_{1}\right)$ and $C\left(I_{F}, d M_{2}\right)$ contains a completely integrable subsystem of rank two that is independent from $I_{F}$.

Recall that for our adapted coframe as in Theorem 3.4, we have

$$
C\left(I_{F}, d M_{1}\right)^{(2)}=\left\{\omega^{4}, \omega^{5}\right\} \quad \text { and } \quad C\left(I_{F}, d M_{2}\right)^{(2)}=\left\{\omega^{6}, \omega^{7}\right\} .
$$

Theorem 7.3. Given a generic hyperbolic PDE $F=0$ with (maximal) 9-dimensional symmetry group, the second derived systems $C\left(I_{F}, d M_{1}\right)^{(2)}$ and $C\left(I_{F}, d M_{2}\right)^{(2)}$ :

1) are completely integrable, and hence $I_{F}$ is Darboux integrable, and

2) contain rank one completely integrable subsystems.

Proof. Referring to the maximally symmetric structure equations (6.5), we have that

$$
d \omega^{4} \equiv d \omega^{5} \equiv 0 \quad \bmod C\left(I_{F}, d M_{1}\right)^{(2)}, \quad d \omega^{6} \equiv d \omega^{7} \equiv 0 \quad \bmod C\left(I_{F}, d M_{2}\right)^{(2)} .
$$

Hence, the rank two systems $C\left(I_{F}, d M_{i}\right)^{(2)}, i=1,2$ are complete integrable and $I_{F}=\left\{\omega^{1}, \omega^{2}\right.$, $\left.\omega^{3}\right\}$ is Darboux integrable. Moreover, since

$$
d \omega^{5}=m \omega^{5} \wedge \omega^{7}, \quad d \omega^{7}=-\frac{\epsilon}{m} \omega^{5} \wedge \omega^{7},
$$

then the rank one subsystems $\left\{\omega^{5}\right\}$ and $\left\{\omega^{7}\right\}$ are also completely integrable. 
Abstractly, Darboux's integration method for these systems proceeds as follows. Darboux integrability of $I_{F}$ implies the existence of completely integrable subsystems $J_{i} \subset C\left(I_{F}, d M_{i}\right)$. Applying the Frobenius theorem to each subsystem $J_{i}$, there exist local functions $f_{i}, g_{i}$ called Riemann invariants such that

$$
J_{1}=\left\{d f_{1}, d g_{1}\right\} \subset C\left(I_{F}, d M_{1}\right), \quad J_{2}=\left\{d f_{2}, d g_{2}\right\} \subset C\left(I_{F}, d M_{2}\right) .
$$

If $\varphi_{1}, \varphi_{2}$ are arbitrary functions, then restricting to any submanifold determined by

$$
S: \quad g_{1}=\varphi_{1}\left(f_{1}\right), \quad g_{2}=\varphi_{2}\left(f_{2}\right),
$$

the structure equations (2.7) become

$$
d \tilde{\omega}^{i} \equiv 0 \quad \bmod \tilde{I}_{F}, \quad i=1,2,3,
$$

where $\tilde{I}_{F}=\left\{\tilde{\omega}^{1}, \tilde{\omega}^{2}, \tilde{\omega}^{3}\right\}$ is the restriction of $I_{F}$ to $S$. Hence, $\tilde{I}_{F}$ is completely integrable, and so there exist local functions $h_{1}, h_{2}, h_{3}$ on $S$ such that

$$
\tilde{I}_{F}=\left\{d h_{1}, d h_{2}, d h_{3}\right\} .
$$

Hence, these functions $h_{1}, h_{2}, h_{3}$ are first integrals of $\tilde{I}_{F}$, and together with the constraint $S$ determine first integrals of $I_{F}$.

Explicitly, from our parametrization of the coframe $\left\{\omega^{i}\right\}_{i=1}^{7}$ on $\Sigma_{7}$ (c.f. (7.1)), we have:

$$
\begin{aligned}
\omega^{4} & =u^{3 / 2} d x+m \sqrt{u}(d y-v d x)=\sqrt{u}(w d x+m d y)=\sqrt{u}(d(m y+w x)-x d w), \\
\omega^{5} & =\frac{\epsilon m}{u}(d u-m d v)=\frac{\epsilon m}{u} d w, \\
\omega^{6} & =-\sqrt{u}(d y-v d x)=-\sqrt{u}(d(y-v x)+x d v), \\
\omega^{7} & =\frac{d v}{u}
\end{aligned}
$$

and so

$$
\begin{aligned}
& C\left(I_{F}, d M_{1}\right)^{(2)}=\left\{\omega^{4}, \omega^{5}\right\}=\{d w, d(m y+w x)\}, \\
& C\left(I_{F}, d M_{2}\right)^{(2)}=\left\{\omega^{6}, \omega^{7}\right\}=\{d v, d(y-v x)\},
\end{aligned}
$$

where $w=u-m v$. Thus,

$$
w, \quad m y+w x, \quad \text { and } \quad v, \quad y-v x
$$

are Riemann invariants and, in principle, Darboux's integration method may be applied to find solutions or first integrals to the original equation. In [11, Corollary 5.9] Gardner-Kamran asserted that hyperbolic equations of generic type do not have Riemann invariants. As first remarked by Eendebak [8], this statement is incorrect and the equation $3 r t^{3}+1=0$ is a counterexample. Moreover, as described above, all maximally symmetric generic hyperbolic equations have Riemann invariants.

We refer the reader to page 130 in Goursat [12] for the implementation of Darboux's method to the equation $3 r t^{3}+1=0$. The implementation of Darboux's method in the case $(\epsilon, a) \neq(1,1)$ appears to be computationally quite difficult.

Let us comment on Darboux integrability for the submaximally symmetric cases described in Table 4. Recall that the structure equations listed in Sections 6.2 and 6.3 are those for the lifted coframe. To obtain the structure equations for the corresponding base coframe, we simply set $\hat{\alpha}^{1}=0$ and remove all hats. For all these cases we have either that

$$
C\left(I_{F}, d M_{1}\right)^{(3)}=\left\{\omega^{4}, \omega^{5}\right\}, \quad C\left(I_{F}, d M_{2}\right)^{(3)}=\left\{\omega^{6}, \omega^{7}\right\}
$$


and hence $I_{F}$ is Darboux integrable, or

$$
C\left(I_{F}, d M_{1}\right)^{(3)}=\left\{\omega^{5}\right\}, \quad C\left(I_{F}, d M_{2}\right)^{(3)}=\left\{\omega^{7}\right\}
$$

and $I_{F}$ is not Darboux integrable. We list the possibilities in Table 5. Moreover, for these submaximally symmetric cases, all which are Darboux integrable have one-dimensional subsystems of $C\left(I_{F}, d M_{1}\right)^{(2)}$ and $C\left(I_{F}, d M_{2}\right)^{(2)}$ which are completely integrable (namely, $\left\{\omega^{5}\right\}$ and $\left\{\omega^{7}\right\}$ respectively). Thus, the converse of Theorem 7.3 is clearly false.

Table 5. Darboux integrability of submaximally symmetric generic hyperbolic PDE.

\begin{tabular}{|c|c|}
\hline Case & Darboux integrable? \\
\hline \hline $2 \mathrm{a}$ & no \\
$2 \mathrm{~b}$ & no in general; yes if $\left(m, \epsilon_{1}\right)=(1,-1)$ \\
\hline 3a & yes \\
$3 \mathrm{~b}$ & no in general; yes if $\left(m, \epsilon_{1}\right)=(1,-1)$ \\
\hline
\end{tabular}

\section{Concluding remarks}

Let us summarize some of the main results of this paper:

- We derived relative invariants $I_{1}, I_{2}$ (see Theorem 3.3) given parametrically in terms of an arbitrary hyperbolic equation $F\left(x, y, z, z_{x}, z_{y}, z_{x x}, z_{x y}, z_{y y}\right)=0$. Their vanishing/nonvanishing distinguishes the three types of hyperbolic equations.

- In the generic case, the $\epsilon$ contact invariant is given parametrically as $\epsilon=\operatorname{sgn}\left(I_{1} I_{2}\right)= \pm 1$.

- In the abstract analysis of the generic hyperbolic structure equations, we identified relative contact invariants $m, n, B$ and $\Delta_{1}=m n+\epsilon, \Delta_{2}=m^{2}-\epsilon n^{2}$ which played a key role in the classification of various generic hyperbolic structures admitting nine, eight, and seven-dimensional symmetry along with the corresponding complete structure equations.

- Integration of maximally symmetric structure equations, leading to normal forms for all contact-equivalence classes of maximally symmetric generic hyperbolic equations.

- Nine-dimensional symmetry algebras for these normal forms for generic hyperbolic equations are given explicitly. There are exactly two such nonisomorphic algebras.

- For any maximally symmetric generic hyperbolic equation, the second derived systems of $C\left(I_{F}, d M_{i}\right), i=1,2$ are rank 2 and completely integrable. Hence, all maximally symmetric generic hyperbolic equations are Darboux integrable.

We conclude with some possible points for future investigation:

1. Maximally symmetric equations: (1) Do "simpler" normal forms exist? (2) Implement Darboux's integration method in the general case. (3) Investigate the existence of conservation laws. (4) Study the local solvability of these equations.

2. Submaximally symmetric equations: Integrate the structure equations given in Sections 6.2 and 6.3 and find normal forms for the corresponding PDE equivalence classes. Address similar questions as above.

3. The submaximally symmetric structures that we have derived here (see Table 4 and Sections 6.2 and 6.3) share the common property that $m, n$ are constants and $K^{0}$ is a subgroup of the structure group. Are there any other reductions of the initial 3-dimensional structure group that lead to valid structures? 
4. In this article, we have carried out a detailed analysis of the generic (7-7) case. Hyperbolic equations of Goursat (6-7) type are equally poorly understood. Some preliminary results on structure equations were stated in [11], but to our knowledge, Vranceanu's student Petrescu [23] has written the only paper which has made a more detailed investigation into the contact geometry of the Goursat class. Recasting Petrescu's results for a contemporary audience and building upon his work would make for a natural sequel to our paper.

\section{A Hyperbolic structure equations}

We give here the details of the proof of Theorem 3.1 starting from the preliminary hyperbolic structure equations (2.7). The main details of this proof have appeared in [25] and [11].

Note A.1. In this section, we will define changes in the coframe basis using a "bar", e.g. $\bar{\omega}^{i}=g_{j}^{i} \omega^{j}$, but we make the convention that the bar is immediately dropped afterwards, i.e. so that $\omega^{i}$ is redefined to be $\bar{\omega}^{i}$.

Proof of Theorem 3.1. From the preliminary hyperbolic structure equations (2.7), we have

$$
d \omega^{1} \equiv \omega^{3} \wedge\left(a_{4} \omega^{4}+a_{5} \omega^{5}+a_{6} \omega^{6}+a_{7} \omega^{7}\right) \quad \bmod M_{1},
$$

but since

$$
\begin{aligned}
& 0=d^{2} \omega^{1} \wedge \omega^{1} \wedge \omega^{2} \wedge \omega^{3} \wedge \omega^{5}=a_{4} \omega^{4} \wedge \omega^{6} \wedge \omega^{7} \wedge \omega^{1} \wedge \omega^{2} \wedge \omega^{3} \wedge \omega^{5}, \\
& 0=d^{2} \omega^{1} \wedge \omega^{1} \wedge \omega^{2} \wedge \omega^{3} \wedge \omega^{4}=a_{5} \omega^{5} \wedge \omega^{6} \wedge \omega^{7} \wedge \omega^{1} \wedge \omega^{2} \wedge \omega^{3} \wedge \omega^{4},
\end{aligned}
$$

then $a_{4}=a_{5}=0$. Assuming $a_{6}=a_{7}=0$, then $d \omega^{1} \equiv 0 \bmod M_{1}$, which would imply that $d \omega^{1} \wedge d \omega^{1} \wedge \omega^{1}=0$, i.e. the Darboux rank $\rho$ of $I_{F}^{(1)}=\left\{\omega^{1}\right\}$ would be $\rho \leq 1$. However, since $\omega^{1}=i_{F}^{*}(d z-p d x-q d y) \in I_{F}^{(1)}$ and $d \omega^{1}=i_{F}^{*}(d x \wedge d p+d y \wedge d q)$, then $d \omega^{1} \wedge d \omega^{1} \wedge \omega^{1}=$ $i_{F}^{*}(d x \wedge d p \wedge d y \wedge d q \wedge d z) \neq 0$ since $i_{F}$ is a nondegenerate parametrization. Hence $\rho \geq 2$ and we get a contradiction. Thus, $a_{6} \omega^{6}+a_{7} \omega^{7} \neq 0$.

Choose $A, B$ such that $B a_{6}-A a_{7}=1$, and redefine

$$
\bar{\omega}^{6}=a_{6} \omega^{6}+a_{7} \omega^{7}, \quad \bar{\omega}^{7}=A \omega^{6}+B \omega^{7} .
$$

This preserves both the $d \omega^{2}$ and $d \omega^{3}$ structure equations (since $\bar{\omega}^{6} \wedge \bar{\omega}^{7}=\omega^{6} \wedge \omega^{7}$ ). We have

$$
d \omega^{1} \equiv \omega^{3} \wedge \omega^{6}, \quad d \omega^{2} \equiv \omega^{4} \wedge \omega^{5}+\omega^{3} \wedge\left(\sum_{k=4}^{7} b_{k} \omega^{k}\right) \quad \bmod M_{1} .
$$

Redefining $\left(\bar{\omega}^{2}, \bar{\omega}^{4}, \bar{\omega}^{5}\right)=\left(\omega^{2}-b_{6} \omega^{1}, \omega^{4}+b_{5} \omega^{3}, \omega^{5}-b_{4} \omega^{3}\right)$, we may without loss of generality take $b_{4}=b_{5}=b_{6}=0$. (Note that the $d \omega^{3}$ equation is not affected.) Setting $b_{7}=U_{1}$, we have

$$
d \omega^{1} \equiv \omega^{3} \wedge \omega^{6}, \quad d \omega^{2} \equiv \omega^{4} \wedge \omega^{5}+U_{1} \omega^{3} \wedge \omega^{7} \quad \bmod M_{1} .
$$

A similar argument modulo $\left\{\omega^{1}, \omega^{3}\right\}$ and for the $M_{2}$ characteristic system yields

$$
d \omega^{1} \equiv \omega^{2} \wedge \omega^{4}, \quad d \omega^{3} \equiv \omega^{6} \wedge \omega^{7}+U_{2} \omega^{2} \wedge \omega^{5} \quad \bmod M_{2},
$$

and we emphasize that $\omega^{i}$ in (A.2) are the same as $\omega^{i}$ in (A.3). This implies

$$
d \omega^{1} \equiv \omega^{2} \wedge \omega^{4}+\omega^{3} \wedge \omega^{6}+W \omega^{2} \wedge \omega^{3} \quad \bmod \left\{\omega^{1}\right\} .
$$

We calculate

$$
\begin{aligned}
& 0=d^{2} \omega^{2} \wedge \omega^{1} \wedge \omega^{2} \wedge \omega^{3} \wedge \omega^{4}=d \omega^{4} \wedge \omega^{5} \wedge \omega^{1} \wedge \omega^{2} \wedge \omega^{3} \wedge \omega^{4} \\
& 0=d^{2} \omega^{1} \wedge \omega^{1} \wedge \omega^{3} \wedge \omega^{4} \wedge \omega^{5}=-\omega^{2} \wedge\left(d \omega^{4}+W \omega^{6} \wedge \omega^{7}\right) \wedge \omega^{1} \wedge \omega^{3} \wedge \omega^{4} \wedge \omega^{5} .
\end{aligned}
$$

The first equation implies that $\gamma^{4}{ }_{67}=0$ (c.f. as defined in (2.8)) and hence the second equation implies $W=0$. 


\section{B Principal contact invariants for hyperbolic equations}

Here we derive the contact invariants stated in Theorem 3.3. We give a brief outline of the computations to follow. Ultimately, we are looking for a coframe satisfying the hyperbolic structure equations given in (3.1). Beginning with the pullback of the basis $\theta^{1}, \theta^{2}, \theta^{3}$ of the contact ideal on $J^{2}\left(\mathbb{R}^{2}, \mathbb{R}\right)$, we find a canonical basis $\tilde{\theta}^{1}, \tilde{\theta}^{2}, \tilde{\theta}^{3}$ whose pullback brings $\langle\cdot, \cdot\rangle_{7}$ into the Witt normal form. This yields the basis $\tilde{\omega}^{1}, \ldots, \tilde{\omega}^{7}$ in Lemma B.2. A subsequent normalization in (B.5) defines the basis $\omega^{1}, \ldots, \omega^{7}$ which satisfies (3.1) and from which (multiples of) $U_{1}$ and $U_{2}$ can be extracted in parametrized form. The final calculation of these parametrized functions is performed in the ambient space $J^{2}\left(\mathbb{R}^{2}, \mathbb{R}\right)$, essentially using the simple fact given in (2.4). Pulling these back by $i_{F}$ yields the desired invariants. We begin with the following lemma.

Lemma B.1. Given a symmetric bilinear form represented by

$$
Q=\left(\begin{array}{cc}
a & -b \\
-b & c
\end{array}\right) \quad \text { with } \quad \Delta=\operatorname{det}(Q)=a c-b^{2}<0,
$$

the change of basis matrix

$$
P=\left(\begin{array}{cc}
\lambda_{+} & c \\
a & \lambda_{+}
\end{array}\right), \quad \lambda_{+}=b+\operatorname{sgn}(b) \sqrt{|\Delta|}, \quad \operatorname{sgn}(x)=\left\{\begin{aligned}
1, & x \geq 0, \\
-1, & x<0,
\end{aligned}\right.
$$

brings $Q$, up to a nonzero scaling, into the Witt normal form, i.e.

$$
P^{t} Q P=\rho\left(\begin{array}{ll}
0 & 1 \\
1 & 0
\end{array}\right), \quad \rho \neq 0 .
$$

Proof. Let $v_{1}, v_{2}$ be the columns of $P$. Note that $\lambda_{ \pm}=b \pm \operatorname{sgn}(b) \sqrt{|\Delta|}$ are roots of the polynomial $\lambda^{2}-2 b \lambda+a c=0$ and that $\lambda_{+} \neq 0$. Hence, $v_{1}, v_{2}$ are nonzero and it is a straightforward verification that $v_{1}$ and $v_{2}$ are null vectors (i.e. $v_{1}^{t} Q v_{1}=v_{2}^{t} Q v_{2}=0$ ). They are linearly independent since $\operatorname{det}(P)=2 \sqrt{|\Delta|}(|b|+\sqrt{|\Delta|}) \neq 0$. Finally, they have nonzero scalar product $\rho=v_{1}^{t} Q v_{2}=2 \Delta \lambda_{+} \neq 0$.

We apply this elementary result to the $2 \times 2$ submatrix appearing in the matrix in (2.5) representing the bilinear form $\langle\cdot, \cdot\rangle_{7}$,

$$
\left(\begin{array}{cc}
F_{t} & -\frac{1}{2} F_{s} \\
-\frac{1}{2} F_{s} & F_{r}
\end{array}\right)
$$

with respect to the basis $\theta^{2}, \theta^{3}$, and arrive at the canonical basis

$$
\tilde{\theta}^{2}=\lambda_{+} \theta^{2}+F_{t} \theta^{3}, \quad \tilde{\theta}^{3}=F_{r} \theta^{2}+\lambda_{+} \theta^{3} .
$$

Without loss of generality, we assume that $F_{s} \geq 0$. (If not, consider the equation $-F=0$ which defines the same locus as $F=0$.) In the notation of the previous lemma, we have

$$
\Delta=F_{t} F_{r}-\frac{1}{4} F_{s}^{2}<0, \quad \lambda_{ \pm}=\frac{F_{s}}{2} \pm \sqrt{|\Delta|} .
$$

Note that $\lambda_{+}>0$, but $\lambda_{-}$may vanish. Both $\lambda_{ \pm}$are roots of the polynomial

$$
\lambda^{2}-F_{s} \lambda+F_{t} F_{r}=0 .
$$


Furthermore, let us note the identities

$$
\lambda_{+} \lambda_{-}=F_{t} F_{r}, \quad \lambda_{+}+\lambda_{-}=F_{s}, \quad \lambda_{+}^{2}-F_{t} F_{r}=2 \lambda_{+} \sqrt{|\Delta|} .
$$

We also define the total derivative operators

$$
D_{x}=\frac{\partial}{\partial x}+p \frac{\partial}{\partial z}+r \frac{\partial}{\partial p}+s \frac{\partial}{\partial q}, \quad D_{y}=\frac{\partial}{\partial y}+q \frac{\partial}{\partial z}+s \frac{\partial}{\partial p}+t \frac{\partial}{\partial q} .
$$

Lemma B.2. Suppose that $F_{s} \geq 0$. Let $\tilde{\omega}^{i}=i_{F}^{*} \tilde{\theta}^{i}$, where

$$
\begin{aligned}
& \tilde{\theta}^{1}=d z-p d x-q d y, \quad \tilde{\theta}^{2}=\lambda_{+} \theta^{2}+F_{t} \theta^{3}, \quad \tilde{\theta}^{3}=F_{r} \theta^{2}+\lambda_{+} \theta^{3}, \\
& \tilde{\theta}^{4}=d x-\frac{F_{r}}{\lambda_{+}} d y, \quad \tilde{\theta}^{5}=\lambda_{+} d r+F_{t} d s+D_{x} F d y, \\
& \tilde{\theta}^{6}=d y-\frac{F_{t}}{\lambda_{+}} d x, \quad \tilde{\theta}^{7}=\lambda_{+} d t+F_{r} d s+D_{y} F d x .
\end{aligned}
$$

Then $\tilde{\boldsymbol{\omega}}=\left\{\tilde{\omega}^{i}\right\}_{i=1}^{7}$ is a (local) coframe on $\Sigma_{7}$ satisfying the structure equations

$$
\begin{array}{rlrl}
d \tilde{\omega}^{1} & \equiv \rho\left(\tilde{\omega}^{2} \wedge \tilde{\omega}^{4}+\tilde{\omega}^{3} \wedge \tilde{\omega}^{6}\right)+\tilde{W} \tilde{\omega}^{2} \wedge \tilde{\omega}^{3} & & \bmod I_{F}^{(1)}, \\
d \tilde{\omega}^{2} \equiv \tilde{\omega}^{4} \wedge \tilde{\omega}^{5}+\tilde{\omega}^{3} \wedge \rho i_{F}^{*} \Xi_{1} & & \bmod M_{1}, \\
d \tilde{\omega}^{3} & \equiv \tilde{\omega}^{6} \wedge \tilde{\omega}^{7}+\tilde{\omega}^{2} \wedge \rho i_{F}^{*} \Xi_{2} & & \bmod M_{2},
\end{array}
$$

where

$$
\begin{aligned}
& I_{F}=\left\{\tilde{\omega}^{1}, \tilde{\omega}^{2}, \tilde{\omega}^{3}\right\}, \quad I_{F}^{(1)}=\left\{\tilde{\omega}^{1}\right\}, \quad \rho=i_{F}^{*}\left(\frac{-1}{2 \sqrt{|\Delta|}}\right), \quad \text { and } \\
& \Xi_{1}=d F_{t}-\frac{F_{t}}{\lambda_{+}} d \lambda_{+}-\left(F_{q}-\frac{F_{t} F_{p}}{\lambda_{+}}\right) d y, \quad \Xi_{2}=d F_{r}-\frac{F_{r}}{\lambda_{+}} d \lambda_{+}-\left(F_{p}-\frac{F_{r} F_{q}}{\lambda_{+}}\right) d x .
\end{aligned}
$$

Proof. We need to show that $\tilde{\boldsymbol{\omega}}$ is linearly independent, or equivalently $\tilde{\omega}^{1} \wedge \cdots \wedge \tilde{\omega}^{7} \neq 0$. By (2.4), it suffices to show that $\tilde{\theta}^{1} \wedge \cdots \wedge \tilde{\theta}^{7} \wedge d F \neq 0$. We calculate

$$
\tilde{\theta}^{1} \wedge \tilde{\theta}^{2} \wedge \tilde{\theta}^{3} \wedge \tilde{\theta}^{4} \wedge \tilde{\theta}^{6}=4|\Delta| d z \wedge d p \wedge d q \wedge d x \wedge d y
$$

and so

$$
\begin{aligned}
& \tilde{\theta}^{1} \wedge \cdots \wedge \tilde{\theta}^{7} \wedge d F \\
& =4|\Delta| d z \wedge d p \wedge d q \wedge d x \wedge d y \wedge\left(\lambda_{+} d t+F_{r} d s\right) \wedge\left(\lambda_{+} d r+F_{t} d s\right) \wedge\left(F_{r} d r+F_{s} d s+F_{t} d t\right) \\
& =4|\Delta| \operatorname{det}\left(\begin{array}{ccc}
0 & F_{r} & \lambda_{+} \\
\lambda_{+} & F_{t} & 0 \\
F_{r} & F_{s} & F_{t}
\end{array}\right) \overbrace{d z \wedge d p \wedge d q \wedge d x \wedge d y \wedge d r \wedge d s \wedge d t}^{\operatorname{Vol}_{J^{2}}\left(\mathbb{R}^{2}, \mathbb{R}\right)} \\
& =4|\Delta| \lambda_{+}\left(-2 F_{t} F_{r}+\lambda_{+} F_{s}\right) \operatorname{Vol}_{J^{2}\left(\mathbb{R}^{2}, \mathbb{R}\right)}=4|\Delta| \lambda_{+}^{2}\left(-2 \lambda_{-}+F_{s}\right) \operatorname{Vol}_{J^{2}\left(\mathbb{R}^{2}, \mathbb{R}\right)} \\
& =8|\Delta|^{3 / 2} \lambda_{+}^{2} \operatorname{Vol}_{J^{2}\left(\mathbb{R}^{2}, \mathbb{R}\right)} \neq 0 .
\end{aligned}
$$

Thus, $\tilde{\boldsymbol{\omega}}$ is a coframe on $\Sigma_{7}$. Let us verify the structure equations. Note that

$$
\begin{array}{lll}
i_{F}^{*} \theta^{2}=\frac{1}{\lambda_{+}^{2}-F_{t} F_{r}}\left(\lambda_{+} \tilde{\omega}^{2}-F_{t} \tilde{\omega}^{3}\right), & i_{F}^{*} \theta^{3}=\frac{1}{\lambda_{+}^{2}-F_{t} F_{r}}\left(-F_{r} \tilde{\omega}^{2}+\lambda_{+} \tilde{\omega}^{3}\right), \\
i_{F}^{*} d x=\frac{\lambda_{+}^{2}}{\lambda_{+}^{2}-F_{t} F_{r}}\left(\tilde{\omega}^{4}+\frac{F_{r}}{\lambda_{+}} \tilde{\omega}^{6}\right), & i_{F}^{*} d y=\frac{\lambda_{+}^{2}}{\lambda_{+}^{2}-F_{t} F_{r}}\left(\frac{F_{t}}{\lambda_{+}} \tilde{\omega}^{4}+\tilde{\omega}^{6}\right),
\end{array}
$$


where all coefficients on the right side are pulled back by $i_{F}^{*}$ to $\Sigma_{7}$ (i.e. evaluated on $F=0$ )

$$
\begin{aligned}
d \tilde{\omega}^{1} & =i_{F}^{*}(d x \wedge d p+d y \wedge d q)=i_{F}^{*}\left(d x \wedge\left(\theta^{2}+s d y\right)+d y \wedge\left(\theta^{3}+s d x\right)\right) \\
& =i_{F}^{*}\left(d x \wedge \theta^{2}+d y \wedge \theta^{3}\right) .
\end{aligned}
$$

Mod $M_{1}$, we have

$$
\begin{aligned}
d \tilde{\omega}^{1} & \equiv \frac{\lambda_{+}^{2}}{\left(\lambda_{+}^{2}-F_{t} F_{r}\right)^{2}}\left(\left(\tilde{\omega}^{4}+\frac{F_{r}}{\lambda_{+}} \tilde{\omega}^{6}\right) \wedge\left(-F_{t} \tilde{\omega}^{3}\right)+\left(\frac{F_{t}}{\lambda_{+}} \tilde{\omega}^{4}+\tilde{\omega}^{6}\right) \wedge\left(\lambda_{+} \tilde{\omega}^{3}\right)\right) \\
& \equiv \frac{\lambda_{+}^{2}}{\left(\lambda_{+}^{2}-F_{t} F_{r}\right)^{2}}\left(\lambda_{+}-\frac{F_{t} F_{r}}{\lambda_{+}}\right) \tilde{\omega}^{6} \wedge \tilde{\omega}^{3} \equiv \frac{\lambda_{+}}{\lambda_{+}^{2}-F_{t} F_{r}} \tilde{\omega}^{6} \wedge \tilde{\omega}^{3} \equiv \frac{-1}{2 \sqrt{|\Delta|}} \tilde{\omega}^{3} \wedge \tilde{\omega}^{6} .
\end{aligned}
$$

$\operatorname{Mod} M_{2}$, we have

$$
\begin{aligned}
d \tilde{\omega}^{1} & \equiv \frac{\lambda_{+}^{2}}{\left(\lambda_{+}^{2}-F_{t} F_{r}\right)^{2}}\left(\left(\tilde{\omega}^{4}+\frac{F_{r}}{\lambda_{+}} \tilde{\omega}^{6}\right) \wedge\left(\lambda_{+} \tilde{\omega}^{2}\right)+\left(\frac{F_{t}}{\lambda_{+}} \tilde{\omega}^{4}+\tilde{\omega}^{6}\right) \wedge\left(-F_{r} \tilde{\omega}^{2}\right)\right) \\
& \equiv \frac{\lambda_{+}^{2}}{\left(\lambda_{+}^{2}-F_{t} F_{r}\right)^{2}}\left(\lambda_{+}-\frac{F_{t} F_{r}}{\lambda_{+}}\right) \tilde{\omega}^{4} \wedge \tilde{\omega}^{2} \equiv \frac{\lambda_{+}}{\lambda_{+}^{2}-F_{t} F_{r}} \tilde{\omega}^{4} \wedge \tilde{\omega}^{2} \equiv \frac{-1}{2 \sqrt{|\Delta|}} \tilde{\omega}^{2} \wedge \tilde{\omega}^{4} .
\end{aligned}
$$

Now examine $d \tilde{\omega}^{2}$ and $d \tilde{\omega}^{3}$. Note the relation

$$
\begin{aligned}
0= & i_{F}^{*}(d F)=i_{F}^{*}\left(F_{x} d x+F_{y} d y+F_{z} d z+F_{p} d p+F_{q} d q+F_{r} d r+F_{s} d s+F_{t} d t\right) \\
= & i_{F}^{*}\left(F_{x} d x+F_{y} d y+F_{z}\left(\theta^{1}+p d x+q d y\right)+F_{p}\left(\theta^{2}+r d x+s d y\right)+F_{q}\left(\theta^{3}+s d x+t d y\right)\right. \\
& \left.+F_{r} d r+F_{s} d s+F_{t} d t\right) \\
= & i_{F}^{*}\left(D_{x} F d x+D_{y} F d y+F_{r} d r+F_{s} d s+F_{t} d t+F_{z} \theta^{1}+F_{p} \theta^{2}+F_{q} \theta^{3}\right)
\end{aligned}
$$

and so,

$$
\begin{array}{lr}
0 \equiv i_{F}^{*}\left(D_{x} F d x+D_{y} F d y+F_{r} d r+F_{s} d s+F_{t} d t+\left(F_{q}-\frac{F_{t} F_{p}}{\lambda_{+}}\right) \theta^{3}\right) & \bmod M_{1}, \\
0 \equiv i_{F}^{*}\left(D_{x} F d x+D_{y} F d y+F_{r} d r+F_{s} d s+F_{t} d t+\left(F_{p}-\frac{F_{r} F_{q}}{\lambda_{+}}\right) \theta^{2}\right) & \bmod M_{2} .
\end{array}
$$

Mod $M_{1}$, we have

$$
\begin{aligned}
d \tilde{\omega}^{2} & \equiv i_{F}^{*}\left(d \lambda_{+} \wedge \theta^{2}+\lambda_{+} d \theta^{2}+F_{t} d \theta^{3}+d F_{t} \wedge \theta^{3}\right) \\
& \equiv i_{F}^{*}\left(-\frac{F_{t}}{\lambda_{+}} d \lambda_{+} \wedge \theta^{3}+\lambda_{+}(d x \wedge d r+d y \wedge d s)+F_{t}(d x \wedge d s+d y \wedge d t)+d F_{t} \wedge \theta^{3}\right) \\
& \equiv i_{F}^{*}\left(\lambda_{+} d x \wedge d r-\lambda_{-} d y \wedge d s+F_{t} d x \wedge d s+D_{x} F d x \wedge d y-F_{r} d y \wedge d r+\Xi_{1} \wedge \theta^{3}\right) \\
& \equiv i_{F}^{*}\left(\left(d x-\frac{F_{r}}{\lambda_{+}} d y\right) \wedge\left(\lambda_{+} d r+F_{t} d s+D_{x} F d y\right)+\Xi_{1} \wedge \theta^{3}\right) \\
& \equiv \tilde{\omega}^{4} \wedge \tilde{\omega}^{5}+\tilde{\omega}^{3} \wedge \rho i_{F}^{*} \Xi_{1} .
\end{aligned}
$$

Mod $M_{2}$, we have

$$
\begin{aligned}
d \tilde{\omega}^{3} & \equiv i_{F}^{*}\left(d F_{r} \wedge \theta^{2}+F_{r} d \theta^{2}+\lambda_{+} d \theta^{3}+d \lambda_{+} \wedge \theta^{3}\right) \\
& \equiv i_{F}^{*}\left(d F_{r} \wedge \theta^{2}+F_{r}(d x \wedge d r+d y \wedge d s)+\lambda_{+}(d x \wedge d s+d y \wedge d t)-\frac{F_{r}}{\lambda_{+}} d \lambda_{+} \wedge \theta^{2}\right) \\
& \equiv i_{F}^{*}\left(F_{r} d y \wedge d s+\lambda_{+}(d x \wedge d s+d y \wedge d t)-d x \wedge\left(D_{y} F d y+F_{s} d s+F_{t} d t\right)+\Xi_{2} \wedge \theta^{2}\right) \\
& \equiv i_{F}^{*}\left(\left(d y-\frac{F_{t}}{\lambda_{+}} d x\right) \wedge\left(\lambda_{+} d t+F_{r} d s+D_{y} F d x\right)+\Xi_{2} \wedge \theta^{2}\right) \\
& \equiv \tilde{\omega}^{6} \wedge \tilde{\omega}^{7}+\tilde{\omega}^{2} \wedge \rho i_{F}^{*} \Xi_{2} .
\end{aligned}
$$


Let us write

$$
\rho i_{F}^{*} \Xi_{1}=b_{4} \tilde{\omega}^{4}+b_{5} \tilde{\omega}^{5}+b_{6} \tilde{\omega}^{6}+b_{7} \tilde{\omega}^{7}, \quad \rho i_{F}^{*} \Xi_{2}=c_{4} \tilde{\omega}^{4}+c_{5} \tilde{\omega}^{5}+c_{6} \tilde{\omega}^{6}+c_{7} \tilde{\omega}^{7} .
$$

We make the change of basis

$$
\begin{aligned}
& \omega^{1}=\tilde{\omega}^{1}, \quad \omega^{2}=\tilde{\omega}^{2}-\frac{b_{6}}{\rho} \tilde{\omega}^{1}, \quad \omega^{3}=\tilde{\omega}^{3}-\frac{c_{4}}{\rho} \tilde{\omega}^{1}, \quad \omega^{4}=\rho\left(\tilde{\omega}^{4}+b_{5} \tilde{\omega}^{3}\right), \\
& \omega^{5}=\frac{1}{\rho}\left(\tilde{\omega}^{5}-b_{4} \tilde{\omega}^{3}\right), \quad \omega^{6}=\rho\left(\tilde{\omega}^{6}+c_{7} \tilde{\omega}^{2}\right), \quad \omega^{7}=\frac{1}{\rho}\left(\tilde{\omega}^{7}-c_{6} \tilde{\omega}^{2}\right)
\end{aligned}
$$

to obtain the structure equations

$$
\begin{array}{rlrl}
d \omega^{1} & \equiv \omega^{2} \wedge \omega^{4}+\omega^{3} \wedge \omega^{6}+W \omega^{2} \wedge \omega^{3} & & \bmod I_{F}^{(1)}, \\
d \omega^{2} \equiv \omega^{4} \wedge \omega^{5}+b_{7} \omega^{3} \wedge \omega^{7} & & \bmod M_{1}, \\
d \omega^{3} & \equiv \omega^{6} \wedge \omega^{7}+c_{5} \omega^{2} \wedge \omega^{5} & & \bmod M_{2},
\end{array}
$$

where $W=\tilde{W}+\rho\left(b_{5}-c_{7}\right)$. Thus, in the notation of Theorem 3.1, we have that $U_{1}=b_{7}$ and $U_{2}=c_{5}$ and by the argument given at the end of Appendix A, we must have that $W=0$.

We are primarily concerned with the vanishing / nonvanishing of $U_{1}$ and $U_{2}$, though the sign will play a later role in the determination of the $\epsilon$ contact invariant. It suffices to calculate

$$
U_{1}: \tilde{\omega}^{7} \text { coefficient in } i_{F}^{*} \Xi_{1}, \quad \text { and } \quad U_{2}: \tilde{\omega}^{5} \text { coefficient in } i_{F}^{*} \Xi_{2} .
$$

Note that the sign of these coefficients is reversed for both since $\rho<0$. As a further simplification, since $i_{F}^{*}(d x), i_{F}^{*}(d y)$ depend only on $\tilde{\omega}^{4}, \tilde{\omega}^{6}$, and since $i_{F}^{*}\left(\lambda_{+}\right)>0$, it suffices to calculate

$$
U_{1}: \tilde{\omega}^{7} \text { coefficient in } i_{F}^{*} d\left(\frac{F_{t}}{\lambda_{+}}\right), \text {and } \quad U_{2}: \tilde{\omega}^{5} \text { coefficient in } i_{F}^{*} d\left(\frac{F_{r}}{\lambda_{+}}\right) .
$$

Let us illustrate how to calculate the $\tilde{\omega}^{7}$ coefficient in $i_{F}^{*} d\left(\frac{F_{t}}{\lambda_{+}}\right)$. Given $\tilde{\omega}=f_{i} \tilde{\omega}^{i}=i_{F}^{*} d\left(\frac{F_{t}}{\lambda_{+}}\right)$ on $\Sigma_{7}$, we are interested in $f_{7}$. Since $i_{F}: \Sigma_{7} \rightarrow J^{2}\left(\mathbb{R}^{2}, \mathbb{R}\right)$ is maximal rank, there exist functions $\tilde{f}_{i}$ on $J^{2}\left(\mathbb{R}^{2}, \mathbb{R}\right)$ such that $i_{F}^{*} \tilde{f}_{i}=f_{i}$, so $\tilde{\omega}=i_{F}^{*}\left(\tilde{f}_{i} \tilde{\theta}^{i}\right)$. Thus, we calculate $\tilde{f}_{7}$ using

$$
\tilde{\theta}^{1} \wedge \cdots \wedge \tilde{\theta}^{6} \wedge d\left(\frac{F_{t}}{\lambda_{+}}\right) \wedge d F=\tilde{f}_{7} \tilde{\theta}^{1} \wedge \cdots \wedge \tilde{\theta}^{7} \wedge d F
$$

and then use $i_{F}^{*}$ to obtain $f_{7}$.

Using (B.3), we have

$$
\begin{aligned}
\nu_{1} & :=\tilde{\theta}^{1} \wedge \tilde{\theta}^{2} \wedge \tilde{\theta}^{3} \wedge \tilde{\theta}^{4} \wedge \tilde{\theta}^{5} \wedge \tilde{\theta}^{6} \wedge d\left(\frac{F_{t}}{\lambda_{+}}\right) \wedge d F \\
& =-4|\Delta| d z \wedge d p \wedge d q \wedge d x \wedge d y \wedge d F \wedge\left(\lambda_{+} d r+F_{t} d s\right) \wedge d\left(\frac{F_{t}}{\lambda_{+}}\right) \\
& =-4|\Delta| \tilde{I}_{1} d z \wedge d p \wedge d q \wedge d x \wedge d y \wedge d r \wedge d s \wedge d t \\
& =\tilde{f}_{7} \tilde{\theta}^{1} \wedge \cdots \wedge \tilde{\theta}^{7} \wedge d F
\end{aligned}
$$

where

$$
\tilde{I}_{1}=\operatorname{det}\left(\begin{array}{ccc}
F_{r} & F_{s} & F_{t} \\
\lambda_{+} & F_{t} & 0 \\
\left(\frac{F_{t}}{\lambda_{+}}\right)_{r} & \left(\frac{F_{t}}{\lambda_{+}}\right)_{s} & \left(\frac{F_{t}}{\lambda_{+}}\right)_{t}
\end{array}\right), \quad \tilde{f}_{7}=\frac{-\tilde{I}_{1}}{2|\Delta|^{1 / 2} \lambda_{+}^{2}} .
$$


Let $I_{1}=i_{F}^{*} \tilde{I}_{1}$, and note $I_{1}$ and $f_{7}=i_{F}^{*} \tilde{f}_{7}$ have opposite sign. Because of the aforementioned sign reversal (i.e. $U_{1}$ and $f_{7}$ have opposite sign), we have

$$
\operatorname{sgn}\left(U_{1}\right)=\operatorname{sgn}\left(I_{1}\right) .
$$

We perform a similar computation for the $\omega^{5}$ coefficient in $i_{F}^{*} d\left(\frac{F_{r}}{\lambda_{+}}\right)$.

$$
\begin{aligned}
\nu_{2} & :=\tilde{\theta}^{1} \wedge \tilde{\theta}^{2} \wedge \tilde{\theta}^{3} \wedge \tilde{\theta}^{4} \wedge d\left(\frac{F_{r}}{\lambda_{+}}\right) \wedge \tilde{\theta}^{6} \wedge \tilde{\theta}^{7} \wedge d F \\
& =-4|\Delta| d z \wedge d p \wedge d q \wedge d x \wedge d y \wedge\left(\lambda_{+} d t+F_{r} d s\right) \wedge d F \wedge d\left(\frac{F_{r}}{\lambda_{+}}\right) \\
& =-4|\Delta| \tilde{I}_{2} d z \wedge d p \wedge d q \wedge d x \wedge d y \wedge d r \wedge d s \wedge d t \\
& =\tilde{f}_{5} \tilde{\theta}^{1} \wedge \cdots \wedge \tilde{\theta}^{7} \wedge d F
\end{aligned}
$$

where

$$
\tilde{I}_{2}=\operatorname{det}\left(\begin{array}{ccc}
0 & F_{r} & \lambda_{+} \\
F_{r} & F_{s} & F_{t} \\
\left(\frac{F_{r}}{\lambda_{+}}\right)_{r} & \left(\frac{F_{r}}{\lambda_{+}}\right)_{s} & \left(\frac{F_{r}}{\lambda_{+}}\right)_{t}
\end{array}\right), \quad \tilde{f}_{5}=\frac{-\tilde{I}_{2}}{2|\Delta|^{1 / 2} \lambda_{+}^{2}} .
$$

Let $I_{2}=i_{F}^{*} \tilde{I}_{2}$, and note $I_{2}$ and $f_{5}=i_{F}^{*} \tilde{f}_{5}$ have opposite sign. Because of the aforementioned sign reversal (i.e. $U_{2}$ and $f_{5}$ have opposite sign), we have

$$
\operatorname{sgn}\left(U_{2}\right)=\operatorname{sgn}\left(I_{2}\right) .
$$

Since class 6-6, 6-7, and 7-7 hyperbolic equations are determined by the vanishing/nonvanishing of $U_{1}, U_{2}$, the classification in Theorem 3.3 follows.

For the scaling property, suppose that $\hat{F}=\phi F$ with $i_{F}^{*} \phi>0$. We may without loss of generality suppose that $\hat{\Sigma}_{7}=\Sigma_{7}$ and $i_{\hat{F}}=i_{F}$. Hence,

$$
\begin{aligned}
& i_{\hat{F}}^{*} \hat{F}_{r}=i_{F}^{*}\left(\phi_{r} F+\phi F_{r}\right)=i_{F}^{*}\left(\phi F_{r}\right), \quad i_{\hat{F}}^{*} \hat{F}_{s}=i_{F}^{*}\left(\phi F_{s}\right), \quad i_{\hat{F}}^{*} \hat{F}_{t}=i_{F}^{*}\left(\phi F_{t}\right), \\
& i_{\hat{F}}^{*} \hat{\Delta}=i_{F}^{*}\left(\phi^{2} \Delta\right), \quad i_{\hat{F}}^{*} \hat{\lambda}_{+}=i_{F}^{*}\left(\phi \lambda_{+}\right) .
\end{aligned}
$$

Since $i_{F}^{*}$ commutes with $d$,

$$
i_{\hat{F}^{*}}^{*} d\left(\frac{\hat{F}_{t}}{\hat{\lambda}_{+}}\right)=i_{F}^{*} d\left(\frac{F_{t}}{\lambda_{+}}\right), \quad i_{\hat{F}}^{*} d\left(\frac{\hat{F}_{r}}{\hat{\lambda}_{+}}\right)=i_{F}^{*} d\left(\frac{F_{r}}{\lambda_{+}}\right) .
$$

From the coframe definition in Lemma B.2, we see that

$$
\left(\hat{\tilde{\omega}}^{1}, \ldots, \hat{\tilde{\omega}}^{7}\right)=\left(\tilde{\omega}^{1},\left(i_{F}^{*} \phi\right) \tilde{\omega}^{2},\left(i_{F}^{*} \phi\right) \tilde{\omega}^{3}, \tilde{\omega}^{4},\left(i_{F}^{*} \phi\right) \tilde{\omega}^{5}, \tilde{\omega}^{6},\left(i_{F}^{*} \phi\right) \tilde{\omega}^{7}\right) .
$$

Hence, from the expression for $\nu_{1}$ in (B.6),

$$
i_{\hat{F}}^{*} \hat{\nu}_{1}=\left(i_{F}^{*} \phi\right)^{4} i_{F}^{*} \nu_{1}
$$

Since $i_{\hat{F}}^{*} \hat{\Delta}=i_{F}^{*}\left(\phi^{2} \Delta\right)$, then by (B.6) and (B.9) we see that necessarily

$$
\hat{I}_{1}=\left(i_{F}^{*} \phi\right)^{2} I_{1} \text {. }
$$

Similarly, we find that

$$
\hat{I}_{2}=\left(i_{F}^{*} \phi\right)^{2} I_{2} .
$$




\section{Generic hyperbolic structure equations}

Starting with the hyperbolic structure equations (c.f. Theorem 3.1), we specialize here to the generic case and prove Theorem 3.4 and Corollaries 3.3 and 3.4.

Proof of Theorem 3.4. Suppose $\operatorname{class}\left(M_{1}\right)=\operatorname{class}\left(M_{2}\right)=7$ so that $U_{1} U_{2} \neq 0$. Let $\left(\bar{\omega}^{1}, \ldots\right.$, $\left.\bar{\omega}^{7}\right)=\left(\lambda_{1} \omega^{1}, \ldots, \lambda_{7} \omega^{7}\right)$, where

$$
\begin{array}{ll}
\lambda_{1}=\left|U_{1}\right|^{-\frac{3}{4}}\left|U_{2}\right|^{-\frac{1}{4}}, & \lambda_{2}=\left|U_{1}\right|^{-\frac{1}{2}}\left|U_{2}\right|^{-\frac{1}{2}}, \quad \lambda_{3}=\left|U_{1}\right|^{-\frac{3}{4}}\left|U_{2}\right|^{-\frac{1}{4}}, \quad \lambda_{4}=\left|U_{1}\right|^{-\frac{1}{4}}\left|U_{2}\right|^{\frac{1}{4}}, \\
\lambda_{5}=\left|U_{1}\right|^{-\frac{1}{4}}\left|U_{2}\right|^{-\frac{3}{4}}, & \lambda_{6}=1, \quad \lambda_{7}=\left|U_{1}\right|^{-\frac{3}{4}}\left|U_{2}\right|^{-\frac{1}{4}} .
\end{array}
$$

This normalizes $\left(U_{1}, U_{2}\right)$ to $\left(\epsilon_{1}, \epsilon_{2}\right)=\left(\operatorname{sgn}\left(U_{1}\right), \operatorname{sgn}\left(U_{2}\right)\right)=( \pm 1, \pm 1)$. Dropping bars and making the further coframe change $\bar{\omega}^{3}=\epsilon_{1} \omega^{3}, \bar{\omega}^{6}=\epsilon_{1} \omega^{6}$ normalizes $\left(\epsilon_{1}, \epsilon_{2}\right)$ to $(1, \epsilon)$, where $\epsilon=\epsilon_{1} \epsilon_{2}=$ \pm 1 . Thus, we have obtained the structure equations (3.2) in Theorem 3.4.

Using the structure equations (3.2), let us now establish explicit generators for $C\left(I_{F}, d M_{1}\right)$ and its derived systems. Let $\left\{\frac{\partial}{\partial \omega^{1}}, \ldots, \frac{\partial}{\partial \omega^{7}}\right\}$ be the dual basis to $\left\{\omega^{1}, \ldots, \omega^{7}\right\}$. From the structure equations, we have

$$
\operatorname{Char}\left(I_{F}, d M_{1}\right)=\left\{\frac{\partial}{\partial \omega^{6}}, \frac{\partial}{\partial \omega^{7}}\right\} \Rightarrow C\left(I_{F}, d M_{1}\right)=\left\{\omega^{1}, \omega^{2}, \omega^{3}, \omega^{4}, \omega^{5}\right\} .
$$

Next, we clearly have $d \omega^{1} \equiv 0, d \omega^{2} \equiv 0, d \omega^{3} \not \equiv 0 \quad \bmod C\left(I_{F}, d M_{1}\right)$ while

$$
\begin{aligned}
& 0=d^{2} \omega^{2} \wedge \omega^{1} \wedge \omega^{2} \wedge \omega^{3} \wedge \omega^{4}=d \omega^{4} \wedge \omega^{5} \wedge \omega^{1} \wedge \omega^{2} \wedge \omega^{3} \wedge \omega^{4}, \\
& 0,=d^{2} \omega^{2} \wedge \omega^{1} \wedge \omega^{2} \wedge \omega^{3} \wedge \omega^{5}=d \omega^{5} \wedge \omega^{4} \wedge \omega^{1} \wedge \omega^{2} \wedge \omega^{3} \wedge \omega^{5}
\end{aligned}
$$

implies that $d \omega^{4} \equiv 0, d \omega^{5} \equiv 0 \bmod C\left(I_{F}, d M_{1}\right)$. Thus, $C\left(I_{F}, d M_{1}\right)^{(1)}=\left\{\omega^{1}, \omega^{2}, \omega^{4}, \omega^{5}\right\}$ and

$$
\begin{array}{rlrl}
d \omega^{1} \equiv \omega^{3} \wedge \omega^{6}, & d \omega^{4} \equiv I \omega^{3} \wedge \omega^{6}+J \omega^{3} \wedge \omega^{7}, \\
d \omega^{2} \equiv \omega^{3} \wedge \omega^{7}, & d \omega^{5} \equiv K \omega^{3} \wedge \omega^{6}+L \omega^{3} \wedge \omega^{7} & \bmod \left\{\omega^{1}, \omega^{2}, \omega^{4}, \omega^{5}\right\} .
\end{array}
$$

We have $d \omega^{1} \not \equiv 0, d \omega^{2} \not \equiv 0 \bmod C\left(I_{F}, d M_{1}\right)^{(1)}$. Redefining

$$
\bar{\omega}^{4}=\omega^{4}-I \omega^{1}-J \omega^{2}, \quad \bar{\omega}^{5}=\omega^{5}-K \omega^{1}-L \omega^{2}
$$

preserves the structure equations (3.2). Dropping bars, we have

$$
d \omega^{4} \equiv 0, \quad d \omega^{5} \equiv 0 \quad \bmod C\left(I_{F}, d M_{1}\right)^{(1)} .
$$

Hence, $C\left(I_{F}, d M_{1}\right)^{(2)}=\left\{\omega^{4}, \omega^{5}\right\}$. Similarly, we describe the derived flag of $C\left(I_{F}, d M_{2}\right)$.

Proof of Corollary 3.3. Using the integrability condition $d^{2} \omega^{1}=0$, we have

$$
\begin{aligned}
& 0=d^{2} \omega^{1} \wedge \omega^{1} \wedge \omega^{2} \wedge \omega^{6}=\left(\omega^{7} \wedge \omega^{4}-d \omega^{6}\right) \wedge \omega^{3} \wedge \omega^{1} \wedge \omega^{2} \wedge \omega^{6}, \\
& 0=d^{2} \omega^{1} \wedge \omega^{1} \wedge \omega^{3} \wedge \omega^{4}=\left(\epsilon \omega^{5} \wedge \omega^{6}-d \omega^{4}\right) \wedge \omega^{2} \wedge \omega^{1} \wedge \omega^{3} \wedge \omega^{4}, \\
& 0=d^{2} \omega^{1} \wedge \omega^{1} \wedge \omega^{4} \wedge \omega^{6}=-\left(\omega^{3} \wedge d \omega^{6}+\omega^{2} \wedge d \omega^{4}\right) \wedge \omega^{1} \wedge \omega^{4} \wedge \omega^{6} .
\end{aligned}
$$

Equation (C.1) implies $\gamma^{6}{ }_{47}=-1$ and $\gamma^{6}{ }_{57}=0$; (C.2) implies $\gamma^{4}{ }_{56}=\epsilon$ and $\gamma^{4}{ }_{57}=0$; (C.3) implies $\gamma^{4}{ }_{35}=\gamma^{6}{ }_{25}$ and $\gamma^{4}{ }_{37}=\gamma^{6}{ }_{27}$. Referring to $C\left(I_{F}, d M_{i}\right)^{(2)}$, in (3.3), we know $\gamma^{6}{ }_{25}=$ $\gamma^{4}{ }_{37}=0$, so we obtain $\gamma^{4}{ }_{35}=\gamma^{6}{ }_{27}=0$. Using these values, we have

$$
0=d^{2} \omega^{4} \wedge \omega^{1} \wedge \omega^{2} \wedge \omega^{4} \wedge \omega^{6}=\left(\gamma^{4}{ }_{25}-\epsilon \gamma^{6}{ }_{37}\right) \wedge \omega^{5} \wedge \omega^{3} \wedge \omega^{7} \wedge \omega^{1} \wedge \omega^{2} \wedge \omega^{4} \wedge \omega^{6} .
$$


Proof of Corollary 3.4. We are interested in all coframe changes which preserve the form of the structure equations (3.2) and (3.4) with the exception of possibly changing the value of the $\epsilon$ coefficient to its negative. If necessarily the value of the $\epsilon$ coefficient is preserved under these coframe changes, then it is a contact invariant. The change of coframe $\bar{\omega}^{i}=R_{j}^{i} \omega^{j}$, where

$$
R=\left(\begin{array}{ccccccc}
-\epsilon & 0 & 0 & 0 & 0 & 0 & 0 \\
0 & 0 & \epsilon & 0 & 0 & 0 & 0 \\
0 & -\epsilon & 0 & 0 & 0 & 0 & 0 \\
0 & 0 & 0 & 0 & 0 & -1 & 0 \\
0 & 0 & 0 & 0 & 0 & 0 & -\epsilon \\
0 & 0 & 0 & 1 & 0 & 0 & 0 \\
0 & 0 & 0 & 0 & -\epsilon & 0 & 0
\end{array}\right)
$$

interchanges the labelling of $M_{1}$ and $M_{2}$ but preserves the structure equations, including the value of $\epsilon$. Thus, without loss of generality, we may restrict to change of coframe which preserve $M_{1}$ and $M_{2}$ and hence each $C\left(I_{F}, d M_{i}\right)^{(k)}$. Thus, we have

$$
\left(\begin{array}{c}
\bar{\omega}^{1} \\
\bar{\omega}^{2} \\
\bar{\omega}^{3} \\
\bar{\omega}^{4} \\
\bar{\omega}^{5} \\
\bar{\omega}^{6} \\
\bar{\omega}^{7}
\end{array}\right)=\left(\begin{array}{ccccccc}
\lambda_{1} & 0 & 0 & 0 & 0 & 0 & 0 \\
\mu_{1} & \lambda_{2} & 0 & 0 & 0 & 0 & 0 \\
\mu_{2} & 0 & \lambda_{3} & 0 & 0 & 0 & 0 \\
0 & 0 & 0 & \lambda_{4} & \nu_{1} & 0 & 0 \\
0 & 0 & 0 & \mu_{3} & \lambda_{5} & 0 & 0 \\
0 & 0 & 0 & 0 & 0 & \lambda_{6} & \nu_{2} \\
0 & 0 & 0 & 0 & 0 & \mu_{4} & \lambda_{7}
\end{array}\right)\left(\begin{array}{c}
\omega^{1} \\
\omega^{2} \\
\omega^{3} \\
\omega^{4} \\
\omega^{5} \\
\omega^{6} \\
\omega^{7}
\end{array}\right) .
$$

Using (3.2) yields

$$
d \bar{\omega}^{1}=d\left(\lambda_{1} \omega^{1}\right) \equiv \lambda_{1}\left(\omega^{3} \wedge \omega^{6}+\omega^{2} \wedge \omega^{4}\right) \quad \bmod I_{F}^{(1)}
$$

and also

$$
d \bar{\omega}^{1} \equiv \bar{\omega}^{3} \wedge \bar{\omega}^{6}+\bar{\omega}^{2} \wedge \bar{\omega}^{4} \equiv \lambda_{3} \omega^{3} \wedge\left(\lambda_{6} \omega^{6}+\nu_{2} \omega^{7}\right)+\lambda_{2} \omega^{2} \wedge\left(\lambda_{4} \omega^{4}+\nu_{1} \omega^{5}\right) \quad \bmod I_{F}^{(1)},
$$

which implies $\nu_{1}=\nu_{2}=0, \lambda_{1}=\lambda_{3} \lambda_{6}=\lambda_{2} \lambda_{4}$. We also have

$$
\begin{aligned}
& d \bar{\omega}^{2}=d\left(\mu_{1} \omega^{1}+\lambda_{2} \omega^{2}\right) \equiv \mu_{1} \omega^{3} \wedge \omega^{6}+\lambda_{2}\left(\omega^{4} \wedge \omega^{5}+\omega^{3} \wedge \omega^{7}\right) \quad \bmod M_{1}, \\
& d \bar{\omega}^{3}=d\left(\mu_{2} \omega^{1}+\lambda_{3} \omega^{3}\right) \equiv \mu_{2} \omega^{2} \wedge \omega^{4}+\lambda_{3}\left(\omega^{6} \wedge \omega^{7}+\epsilon \omega^{2} \wedge \omega^{5}\right) \quad \bmod M_{2}
\end{aligned}
$$

and

$$
\begin{aligned}
& d \bar{\omega}^{2} \equiv \bar{\omega}^{4} \wedge \bar{\omega}^{5}+\bar{\omega}^{3} \wedge \bar{\omega}^{7} \equiv \lambda_{4} \omega^{4} \wedge \lambda_{5} \omega^{5}+\lambda_{3} \omega^{3} \wedge\left(\mu_{4} \omega^{6}+\lambda_{7} \omega^{7}\right) \quad \bmod M_{1}, \\
& d \bar{\omega}^{3} \equiv \bar{\omega}^{6} \wedge \bar{\omega}^{7}+\delta \bar{\omega}^{2} \wedge \bar{\omega}^{5} \equiv \lambda_{6} \omega^{6} \wedge \lambda_{7} \omega^{7}+\delta \lambda_{2} \omega^{2} \wedge\left(\mu_{3} \omega^{4}+\lambda_{5} \omega^{5}\right) \quad \bmod M_{2},
\end{aligned}
$$

where $\delta= \pm \epsilon$. Consequently, we have the system of equations

$$
\begin{array}{ll}
\lambda_{1}=\lambda_{3} \lambda_{6}=\lambda_{2} \lambda_{4}, & \nu_{1}=\nu_{2}=0, \\
\lambda_{2}=\lambda_{4} \lambda_{5}=\lambda_{3} \lambda_{7}, & \mu_{1}=\lambda_{3} \mu_{4}, \\
\lambda_{3}=\lambda_{6} \lambda_{7}=\delta \epsilon \lambda_{2} \lambda_{5}, & \mu_{2}=\delta \lambda_{2} \mu_{3} .
\end{array}
$$

Then

$$
\beta:=\frac{\lambda_{6}}{\lambda_{4}}=\frac{\lambda_{2}}{\lambda_{3}}=\frac{\delta \epsilon}{\lambda_{5}}=\frac{\delta \epsilon \lambda_{4}}{\lambda_{2}}, \quad \beta=\frac{\lambda_{2}}{\lambda_{3}}=\lambda_{7}=\frac{\lambda_{3}}{\lambda_{6}},
$$


and so

$$
0 \leq \beta^{4}=\left(\frac{\lambda_{6}}{\lambda_{4}}\right)\left(\frac{\lambda_{2}}{\lambda_{3}}\right)\left(\frac{\delta \epsilon \lambda_{4}}{\lambda_{2}}\right)\left(\frac{\lambda_{3}}{\lambda_{6}}\right)=\delta \epsilon .
$$

Consequently, we must have $\delta=\epsilon$ and so $\epsilon$ is a contact invariant.

Finally, from (B.7), (B.8), and the proof of Theorem 3.4 given in Appendix C, we have

$$
\epsilon=\operatorname{sgn}\left(U_{1} U_{2}\right)=\operatorname{sgn}\left(I_{1} I_{2}\right) .
$$

Remark C.1. Vranceanu [25] incorrectly asserts that $\epsilon$ can be normalized to 1 . In particular, in the formulas preceding his equation (4) he writes $\nu=\left(B C^{3}\right)^{\frac{1}{4}}$. This is invalid if $B C<0$.

\section{Isolating maximally and submaximally symmetric structures}

Starting with the structure equations (3.2), (3.4) (and not assuming $\gamma^{5}{ }_{56}=0$ ) we clarify Vranceanu's method of isolating all structures admitting maximal symmetry as well as several structures admitting submaximal symmetry. The key to understanding Vranceanu's method occurs in a single rather cryptic paragraph on pages 367-368 of [25]. In our notation, Vranceanu writes:

In effect, we can remark that if $a_{1}$ were equal to 1 we could reduce $a_{2}$, and consequently $a_{3}$, to zero, by cancelling in the covariant $d \omega^{6}$ the term $\gamma^{6}{ }_{46}$ with the help of the coefficient $a_{2}$. This indicates that for $a_{2}, a_{3}$ different than zero, we must have $a_{1} \neq 1$, and consequently the systems for which we cannot reduce $a_{2}$ and $a_{3}$ to zero are found amongst those for which $a_{1} \neq 1$, $a_{2}=a_{3}=0$, and such that we cannot reduce $a_{1}$ to 1 .

Let us refer back to the structure equations (5.2) for the lifted coframe on $\Sigma_{7} \times G \rightarrow \Sigma_{7}$. Let $H^{0}$ denote the subgroup obtained by setting $a_{1}=1$ in $G^{0}$. Since Vranceanu did not consider discrete symmetries, let us consider the corresponding lifted coframe on $\Sigma_{7} \times H^{0} \rightarrow \Sigma_{7}$. We would have structure equations as in (5.2) except no $\alpha^{1}$ terms would appear (and $d \alpha^{2}=d \alpha^{3}=0$ ). Thus, using Lie algebra valued compatible absorption using $\alpha^{2}$ and $\alpha^{3}$ only, $\gamma^{6} 46$ would be a torsion coefficient. With respect to the group $H^{0}$, we have the transformation laws

$$
\hat{\gamma}_{46}^{6}=\gamma^{6}{ }_{46}-\gamma^{6}{ }_{56} a_{3}+a_{2}, \quad \hat{\gamma}^{6}{ }_{56}=\gamma^{6}{ }_{56} .
$$

By setting $a_{2}=\gamma^{6}{ }_{56} a_{3}-\gamma^{6}{ }_{46}$, we can normalize $\hat{\gamma}_{46}^{6}=0$. In the group reduced from $H^{0}$, we only obtain $a_{2}=\gamma^{6}{ }_{56} a_{3}$, so it is unclear whether $a_{2}, a_{3}$ can both be reduced to 0 . Thus, Vranceanu's claim may be true, but if so it is certainly not obvious. Are there any structures for which $a_{1}$ can be reduced to 1 , but at least one of $a_{2}, a_{3}$ are nonzero?

The second sentence in the paragraph appears to be quite cryptic and at first sight appears even self-contradictory. One can make sense of this as follows: Vranceanu sets out to find all structures for which $K^{0}=\left\{\operatorname{diag}\left(a_{1}^{2}, a_{1}, a_{1}, a_{1}, 1, a_{1}, 1\right): a_{1}>0\right\} \subset G$ cannot be reduced to the identity. Thus, he is considering the restricted equivalence problem with respect to the subgroup $K^{0}$, and a lifted coframe on the bundle $\Sigma_{7} \times K^{0} \rightarrow \Sigma_{7}$ satisfying the structure equations

$$
\begin{aligned}
& d \hat{\omega}^{1}=2 \alpha^{1} \wedge \hat{\omega}^{1}+\hat{\omega}^{3} \wedge \hat{\omega}^{6}+\hat{\omega}^{2} \wedge \hat{\omega}^{4}+\eta_{1} \wedge \hat{\omega}^{1}, \\
& d \hat{\omega}^{2}=\alpha^{1} \wedge \hat{\omega}^{2}+\hat{\omega}^{4} \wedge \hat{\omega}^{5}+\hat{\omega}^{3} \wedge \hat{\omega}^{7}+\eta_{21} \wedge \hat{\omega}^{1}+\eta_{22} \wedge \hat{\omega}^{2}, \\
& d \hat{\omega}^{3}=\alpha^{1} \wedge \hat{\omega}^{3}+\hat{\omega}^{6} \wedge \hat{\omega}^{7}+\epsilon \hat{\omega}^{2} \wedge \hat{\omega}^{5}+\eta_{31} \wedge \hat{\omega}^{1}+\eta_{33} \wedge \hat{\omega}^{3}, \\
& d \hat{\omega}^{4}=\alpha^{1} \wedge \hat{\omega}^{4}+\eta_{41} \wedge \hat{\omega}^{1}+\eta_{42} \wedge \hat{\omega}^{2}+\eta_{44} \wedge \hat{\omega}^{4}+\epsilon \hat{\omega}^{5} \wedge \hat{\omega}^{6}, \\
& d \hat{\omega}^{5}=\eta_{51} \wedge \hat{\omega}^{1}+\eta_{52} \wedge \hat{\omega}^{2}+\eta_{54} \wedge \hat{\omega}^{4}+\eta_{55} \wedge \hat{\omega}^{5}, \\
& d \hat{\omega}^{6}=\alpha^{1} \wedge \hat{\omega}^{6}+\eta_{61} \wedge \hat{\omega}^{1}+\eta_{63} \wedge \hat{\omega}^{3}+\eta_{66} \wedge \hat{\omega}^{6}-\hat{\omega}^{4} \wedge \hat{\omega}^{7},
\end{aligned}
$$




$$
\begin{aligned}
& d \hat{\omega}^{7}=\eta_{71} \wedge \hat{\omega}^{1}+\eta_{73} \wedge \hat{\omega}^{3}+\eta_{76} \wedge \hat{\omega}^{6}+\eta_{77} \wedge \hat{\omega}^{7}, \\
& d \alpha^{1}=0 .
\end{aligned}
$$

where $\eta_{i}$ are semibasic with respect to the canonical projection $\Sigma_{7} \times K^{0} \rightarrow \Sigma_{7}$. Note that under the $K^{0}$-action, all coefficients transform by a scaling action:

$$
\hat{\gamma}_{j k}^{i}=\left(a_{1}\right)^{p} \gamma_{j k}^{i}
$$

for some scaling weight $p \in \mathbb{R}$. (In other words, by setting $a_{2}=a_{3}=0$ in the general transformation formulas under $G^{0}$, c.f. last sentence in Vranceanu's paragraph above.) For specific $i, j, k$, if $\gamma^{i}{ }_{j k} \neq 0$, then we can normalize to $\hat{\gamma}^{i}{ }_{j k}= \pm 1$ by setting $a_{1}=\left|\gamma_{j k}^{i}\right|^{-1 / p}$, thereby reducing $K^{0}$ to the identity. Since we are assuming that $K^{0}$ cannot be reduced, Vranceanu's conclusion would be that $\gamma^{i}{ }_{j k}=0$. A technical assumption that should be made here is that if $p \neq 0$, we are considering $\gamma^{i}{ }_{j k}$ to be a constant torsion coefficient. For example, if $\gamma_{j k}^{i} \neq 0$ (as functions) but vanishes at a point, then $K$ cannot be normalized since $a_{1}$ must be nonzero. In summary, we have:

Lemma D.1. Suppose $\hat{\kappa}=\left(a_{1}\right)^{p} \kappa$ is a torsion coefficient with respect to $K^{0}$. If (1) $K^{0}$ cannot be reduced to the identity, (2) $p \neq 0$, and (3) $\kappa$ is a constant, then $\kappa=0$.

Note D.1. We do not need to assume that all torsion terms are constant. Only those torsion terms with a nontrivial scaling action by $K^{0}$ are assumed to be constant.

Vranceanu finishes the paragraph with the consequences of this simplification:

However, it is easy to see that for these systems we must have in the formulas (3.4), all null coefficients, except for $\gamma^{4}{ }_{25}, \gamma^{4}{ }_{27}, \gamma^{4}{ }_{53}, \gamma^{4}{ }_{56}=1, \gamma_{57}^{5}, \gamma^{6}{ }_{35}, \gamma^{6}{ }_{37}, \gamma_{72}^{6}, \gamma^{6}{ }_{74}=1, \gamma^{7}{ }_{75}$ and likewise one must have $\gamma^{2}{ }_{3 \alpha}=0(\alpha \neq 2), \gamma^{3}{ }_{2 \alpha}=0(\alpha \neq 3)$.

Note D.2. The values $\gamma^{4}{ }_{53}=\gamma^{6}{ }_{72}=0, \gamma^{4}{ }_{56}=\epsilon, \gamma^{6}{ }_{47}=-1$ were established in Corollary 3.3. Also, the final assertions in Vranceanu's sentence above should read $\gamma^{2}{ }_{1 \alpha}$ and $\gamma^{3}{ }_{1 \alpha}$ respectively see explanations below.

We give an outline of these details. With respect to the subgroup $K^{0}$, the following are torsion terms (c.f. (D.2)):

$$
\begin{array}{lcccccc}
\hat{\gamma}^{2}{ }_{1 k} & (k \neq 2), \quad \hat{\gamma}^{3}{ }_{1 k} \quad(k \neq 3), & \hat{\gamma}^{4}{ }_{1 k}, \hat{\gamma}_{2 k}^{4} & (k \neq 4), & \hat{\gamma}^{6}{ }_{1 k}, \hat{\gamma}^{6}{ }_{3 k} & (k \neq 6), \\
\hat{\gamma}^{5}{ }_{1 k}, & \hat{\gamma}^{5}{ }_{2 k}, \hat{\gamma}^{5}{ }_{4 k}, & \hat{\gamma}^{7}{ }_{1 k}, \hat{\gamma}^{7}{ }_{3 k}, \hat{\gamma}_{6 k}^{7} & (k \text { arbitrary }), & \hat{\gamma}^{5}{ }_{35}, \hat{\gamma}_{27}^{7}, \hat{\gamma}^{7}{ }_{47} .
\end{array}
$$

The scaling weight for all these terms is nonzero with the exception of $\hat{\gamma}^{4}{ }_{25}, \hat{\gamma}^{4}{ }_{27}, \hat{\gamma}^{6}{ }_{35}, \hat{\gamma}^{6}{ }_{37}$ for which $p=0$. By Lemma D.1, all of the corresponding coefficients must vanish with the exception of $\gamma^{4}{ }_{25}, \gamma^{4}{ }_{27}, \gamma^{6}{ }_{35}, \gamma^{6}{ }_{37}$. Vranceanu did not make use of the following torsion terms:

$$
\begin{array}{ccccc}
2 \hat{\gamma}_{k 2}^{2}-\hat{\gamma}_{k 1}^{1} & (k \neq 1,2), & 2 \hat{\gamma}_{k 3}^{3}-\hat{\gamma}_{k 1}^{1} & (k \neq 1,3), & 2 \hat{\gamma}_{k 4}^{4}-\hat{\gamma}_{k 1} \quad(k \neq 1,4), \\
2 \hat{\gamma}_{k 6}^{6}-\hat{\gamma}_{k 1}^{1} & (k \neq 1,6), & \hat{\gamma}^{3}{ }_{13}-\hat{\gamma}_{12}^{2}, & \hat{\gamma}^{4}{ }_{14}-\hat{\gamma}_{12}^{2}, & \hat{\gamma}_{16}^{6}-\hat{\gamma}_{12}^{2} .
\end{array}
$$

All of these terms have nonzero scaling weight (and hence must vanish) with the exception of

$$
2 \hat{\gamma}_{k i}^{i}-\hat{\gamma}_{k 1}^{1} \quad \text { (no sum), } \quad i=2,3,4,6, \quad k=5,7 .
$$

Thus, the structure equations take the form

$$
\begin{aligned}
& d \omega^{1}=\omega^{3} \wedge \omega^{6}+\omega^{2} \wedge \omega^{4}+\omega^{1} \wedge \eta_{1}, \\
& d \omega^{2}=\omega^{4} \wedge \omega^{5}+\omega^{3} \wedge \omega^{7}+\omega^{2} \wedge \eta_{2},
\end{aligned}
$$




$$
\begin{aligned}
& d \omega^{3}=\omega^{6} \wedge \omega^{7}+\epsilon \omega^{2} \wedge \omega^{5}+\omega^{3} \wedge \eta_{3}, \\
& d \omega^{4}=\epsilon \omega^{5} \wedge \omega^{6}+\omega^{2} \wedge\left(\gamma^{4}{ }_{25} \omega^{5}+\gamma^{4}{ }_{27} \omega^{7}\right)+\omega^{4} \wedge \eta_{4}, \\
& d \omega^{5}=m \omega^{5} \wedge \omega^{7}, \\
& d \omega^{6}=-\omega^{4} \wedge \omega^{7}+\omega^{3} \wedge\left(\gamma^{6}{ }_{35} \omega^{5}+\gamma^{6}{ }_{37} \omega^{7}\right)+\omega^{6} \wedge \eta_{6}, \\
& d \omega^{7}=n \omega^{5} \wedge \omega^{7},
\end{aligned}
$$

where $\eta_{k}=\gamma_{k \ell}^{k} \omega^{\ell}$ (no sum on $k$ ), and

$$
\begin{array}{ll}
2 \gamma^{4}{ }_{46}=2 \gamma^{3}{ }_{36}=2 \gamma^{2}{ }_{26}=\gamma_{16}^{1}, & 2 \gamma_{63}^{6}=2 \gamma^{4}{ }_{43}=2 \gamma^{2}{ }_{23}=\gamma_{13}^{1}, \\
2 \gamma^{6}{ }_{64}=2 \gamma^{3}{ }_{34}=2 \gamma^{2}{ }_{24}=\gamma_{14}^{1}, & 2 \gamma^{6}{ }_{62}=2 \gamma^{4}{ }_{42}=2 \gamma^{3}{ }_{32}=\gamma_{12}^{1}, \\
\gamma^{6}{ }_{16}=\gamma^{4}{ }_{14}=\gamma_{13}^{3}=\gamma_{12}^{2}, &
\end{array}
$$

or more succinctly,

$$
\begin{aligned}
\gamma_{k \ell}^{k} & =\frac{1}{2} \gamma_{1 \ell}^{1} \quad(\text { no sum }), \quad k, \ell=2,3,4,6, \\
\gamma^{k}{ }_{1 k} & =\gamma^{2}{ }_{12} \quad(\text { no sum }), \quad k=3,4,6 .
\end{aligned}
$$

Lemma D.2. There exists an admissible change of coframe so that $\eta_{1}=0$ and $\gamma^{2}{ }_{12}=0$.

Proof. Let us evaluate some integrability conditions.

$$
0=d^{2} \omega^{1}=\left(\eta_{1}-\eta_{2}-\eta_{4}\right) \wedge \omega^{2} \wedge \omega^{4}+\left(\eta_{1}-\eta_{3}-\eta_{6}\right) \wedge \omega^{3} \wedge \omega^{6}-\omega^{1} \wedge d \eta_{1} .
$$

Isolating terms that are in the ideal generated by $\omega^{1}$ and using (D.5), we have

$$
0=\omega^{1} \wedge\left(2 \gamma^{2}{ }_{12} d \omega^{1}-d \eta_{1}\right)
$$

Note that for $k=2,3,4,6$,

$$
\begin{aligned}
0 & \left.=d^{2} \omega^{k} \wedge \omega^{5} \wedge \omega^{7}=-\omega^{k} \wedge d \eta_{k} \wedge \omega^{5} \wedge \omega^{7} \quad \text { (no sum on } k\right) \\
& =-\omega^{k} \wedge d\left(\gamma_{k \ell}^{k} \omega^{\ell}\right) \wedge \omega^{5} \wedge \omega^{7}=-\omega^{k} \wedge d\left(\gamma^{2}{ }_{21} \omega^{1}+\frac{1}{2} \eta_{1}\right) \wedge \omega^{5} \wedge \omega^{7} \\
& =-\omega^{k} \wedge\left(d \gamma_{21}^{2} \wedge \omega^{1}+\gamma_{21}^{2} d \omega^{1}+\frac{1}{2} d \eta_{1}\right) \wedge \omega^{5} \wedge \omega^{7}
\end{aligned}
$$

Thus, (D.7) and (D.8) imply

$$
d \eta_{1}=d\left(2 \gamma^{2}{ }_{12} \omega^{1}\right)+\omega^{1} \wedge\left(f_{1} \omega^{5}+f_{2} \omega^{7}\right)
$$

for some functions $f, g$. Applying $d$ and wedging with $\omega^{1}$ yields

$$
0=\omega^{1} \wedge d \omega^{1} \wedge\left(f_{1} \omega^{5}+f_{2} \omega^{7}\right)=\omega^{1} \wedge\left(\omega^{2} \wedge \omega^{4}+\omega^{3} \wedge \omega^{6}\right) \wedge\left(f_{1} \omega^{5}+f_{2} \omega^{7}\right)
$$

and so we must have $f_{1}=f_{2}=0$. By Poincaré's lemma, we have

$$
\eta_{1}=2 \gamma^{2}{ }_{12} \omega^{1}+2 d h
$$

for some function $h$. Define the change of coframe

$$
\left(\bar{\omega}^{1}, \bar{\omega}^{2}, \bar{\omega}^{3}, \bar{\omega}^{4}, \bar{\omega}^{6}\right)=\left(a_{1}{ }^{2} \omega^{1}, a_{1} \omega^{2}, a_{1} \omega^{3}, a_{1} \omega^{4}, a_{1} \omega^{6}\right), \quad \text { where } \quad a_{1}=e^{h} .
$$


Then

$$
\begin{aligned}
d \bar{\omega}^{1} & =2 e^{2 h} d h \wedge \omega^{1}+e^{2 h} d \omega^{1}=2 e^{2 h} d h \wedge \omega^{1}+e^{2 h}\left(\omega^{2} \wedge \omega^{4}+\omega^{3} \wedge \omega^{6}+\omega^{1} \wedge \eta_{1}\right) \\
& =2 d h \wedge \bar{\omega}^{1}+\bar{\omega}^{2} \wedge \bar{\omega}^{4}+\bar{\omega}^{3} \wedge \bar{\omega}^{6}+\bar{\omega}^{1} \wedge \eta_{1} \\
& =\bar{\omega}^{2} \wedge \bar{\omega}^{4}+\bar{\omega}^{3} \wedge \bar{\omega}^{6}
\end{aligned}
$$

Dropping bars, we have a new coframe satisfying (D.3) with $\eta_{1}=0$. Moreover, using (D.7), we have that $\gamma_{12}^{2}=0$.

From (D.6), we have that

$$
\gamma_{45}^{4}=-\gamma_{25}^{2}, \quad \gamma_{47}^{4}=-\gamma_{27}^{2}, \quad \gamma_{65}^{6}=-\gamma^{3}{ }_{35}, \quad \gamma_{67}^{6}=-\gamma^{3}{ }_{37},
$$

and using (D.4), (D.5), the structure equations take the form

$$
\begin{aligned}
& d \omega^{1}=\omega^{3} \wedge \omega^{6}+\omega^{2} \wedge \omega^{4}, \\
& d \omega^{2}=\omega^{4} \wedge \omega^{5}+\omega^{3} \wedge \omega^{7}+\omega^{2} \wedge\left(\gamma^{2}{ }_{25} \omega^{5}+\gamma^{2}{ }_{27} \omega^{7}\right), \\
& d \omega^{3}=\omega^{6} \wedge \omega^{7}+\epsilon \omega^{2} \wedge \omega^{5}+\omega^{3} \wedge\left(\gamma^{3}{ }_{35} \omega^{5}+\gamma^{3}{ }_{37} \omega^{7}\right), \\
& d \omega^{4}=\epsilon \omega^{5} \wedge \omega^{6}+\omega^{2} \wedge\left(\gamma^{4}{ }_{25} \omega^{5}+\gamma^{4}{ }_{27} \omega^{7}\right)-\omega^{4} \wedge\left(\gamma^{2}{ }_{25} \omega^{5}+\gamma^{2}{ }_{27} \omega^{7}\right), \\
& d \omega^{5}=m \omega^{5} \wedge \omega^{7}, \\
& d \omega^{6}=-\omega^{4} \wedge \omega^{7}+\omega^{3} \wedge\left(\gamma^{6}{ }_{35} \omega^{5}+\gamma^{6}{ }_{37} \omega^{7}\right)-\omega^{6} \wedge\left(\gamma^{3}{ }_{35} \omega^{5}+\gamma^{3}{ }_{37} \omega^{7}\right), \\
& d \omega^{7}=n \omega^{5} \wedge \omega^{7} .
\end{aligned}
$$

Further integrability conditions reveal

$$
\begin{aligned}
& 0=d^{2} \omega^{5}=d m \wedge \omega^{5} \wedge \omega^{7} \quad \Rightarrow \quad d m=m_{5} \omega^{5}+m_{7} \omega^{7}, \\
& 0=d^{2} \omega^{7}=d n \wedge \omega^{5} \wedge \omega^{7} \quad \Rightarrow \quad d n=n_{5} \omega^{5}+n_{7} \omega^{7} .
\end{aligned}
$$

Next,

$$
\begin{aligned}
& 0=d^{2} \omega^{2} \wedge \omega^{2}=\left(n-\gamma_{35}^{3}+\gamma_{25}^{2}\right) \omega^{2} \wedge \omega^{3} \wedge \omega^{5} \wedge \omega^{7}+\left(m-2 \gamma^{2}{ }_{27}\right) \omega^{2} \wedge \omega^{4} \wedge \omega^{5} \wedge \omega^{7}, \\
& 0=d^{2} \omega^{3} \wedge \omega^{3}=\epsilon\left(-m+\gamma^{3}{ }_{37}-\gamma^{2}{ }_{27}\right) \omega^{2} \wedge \omega^{3} \wedge \omega^{5} \wedge \omega^{7}-\left(n+2 \gamma^{3} 35\right) \omega^{3} \wedge \omega^{5} \wedge \omega^{6} \wedge \omega^{7}, \\
\Rightarrow & \gamma^{2}{ }_{25}=-\frac{3 n}{2}, \quad \gamma_{27}^{2}=\frac{m}{2}, \quad \gamma^{3}{ }_{35}=-\frac{n}{2}, \quad \gamma^{3} 37=\frac{3 m}{2}, \\
& 0=d^{2} \omega^{2}=\left(m n+\epsilon-\gamma^{4}{ }_{27}\right) \omega^{2} \wedge \omega^{5} \wedge \omega^{7}+\frac{1}{2} \omega^{2} \wedge\left(3 d n \wedge \omega^{5}-d m \wedge \omega^{7}\right), \\
& 0=d^{2} \omega^{3}=\left(\gamma^{6}{ }_{35}-m n-\epsilon\right) \omega^{3} \wedge \omega^{5} \wedge \omega^{7}+\frac{1}{2} \omega^{3} \wedge\left(d n \wedge \omega^{5}-3 d m \wedge \omega^{7}\right), \\
& 0=d^{2} \omega^{4} \wedge \omega^{1} \wedge \omega^{2} \wedge \omega^{4} \wedge \omega^{6}=\left(\epsilon \gamma^{6}{ }_{37}-\gamma^{4}{ }_{25}\right) \omega^{3} \wedge \omega^{5} \wedge \omega^{7} \wedge \omega^{1} \wedge \omega^{2} \wedge \omega^{4} \wedge \omega^{6}, \\
\Rightarrow & \gamma_{25}^{4}=B=\epsilon \gamma_{37}^{6}, \quad \gamma^{4}{ }_{27}=m n+\epsilon-\frac{3}{2} n_{7}-\frac{1}{2} m_{5}, \quad \gamma_{35}^{6}=m n+\epsilon+\frac{1}{2} n_{7}+\frac{3}{2} m_{5} .
\end{aligned}
$$

The final two integrability conditions are

$$
\begin{aligned}
0=d^{2} \omega^{4}= & \left(-4 n \Delta_{1}-2 m B+6 n n_{7}+n m_{5}-m n_{5}\right) \omega^{2} \wedge \omega^{5} \wedge \omega^{7}+\omega^{2} \wedge \omega^{5} \wedge d B \\
& -\frac{1}{2} \omega^{2} \wedge \omega^{7} \wedge\left(d m_{5}+3 d n_{7}\right), \\
0=d^{2} \omega^{6}= & \left(-4 m \Delta_{1}-2 n \epsilon B-6 m m_{5}-m n_{7}+n m_{7}\right) \omega^{3} \wedge \omega^{5} \wedge \omega^{7}+\epsilon \omega^{3} \wedge \omega^{7} \wedge d B \\
& +\frac{1}{2} \omega^{3} \wedge \omega^{5} \wedge\left(3 d m_{5}+d n_{7}\right),
\end{aligned}
$$


which implies

$$
\begin{aligned}
& 0=d^{2} \omega^{4} \wedge \omega^{7}=\omega^{2} \wedge \omega^{5} \wedge d B \wedge \omega^{7}, \\
& 0=d^{2} \omega^{6} \wedge \omega^{5}=\epsilon \omega^{3} \wedge \omega^{7} \wedge d B \wedge \omega^{5},
\end{aligned}
$$

and hence $d^{2} \omega^{4}=d^{2} \omega^{6}=0$ is equivalent to

$$
\begin{aligned}
d B= & \epsilon\left(-4 m \Delta_{1}-2 n \epsilon B-6 m m_{5}-m n_{7}+n m_{7}+\frac{3}{2} m_{57}+\frac{1}{2} n_{77}\right) \omega^{5} \\
& +\left(4 n \Delta_{1}+2 m B-6 n n_{7}-n m_{5}+m n_{5}-\frac{1}{2} m_{55}-\frac{3}{2} n_{75}\right) \omega^{7} .
\end{aligned}
$$

This reduces the structure equations to those given in Theorem 6.1.

\section{E Parametrization of maximally symmetric structures}

While in general it is very difficult to go from structure equations specified in an abstract coframe to a coframe described parametrically (i.e. in local coordinates), Vranceanu succeeded in doing this for the maximally symmetric structure equations (6.5). Certain steps in his computation were unjustified or contained errors (e.g. Vranceanu missed the contact invariant $\epsilon$ in his structure equations and hence his normal forms), and so we provide a more complete outline here.

Let us consider a coframe $\left\{\omega^{i}\right\}_{i=1}^{7}$ on $\Sigma_{7}$ satisfying (6.5), and let us consider $\omega^{1}$ in its canonical form

$$
\omega^{1}=d z-p d x-q d y
$$

\section{$\omega^{5}$ and $\omega^{7}$}

The subsystem $\left\{\omega^{5}, \omega^{7}\right\}$ is completely integrable, so by the Frobenius theorem there exist local functions $u, v$ on $\Sigma_{7}$ such that $\{d u, d v\}$ generate the same subsystem. We introduce $u, v$ as follows. By using the $d \omega^{5}$ and $d \omega^{7}$ equations,

$$
0=d\left(\frac{\epsilon}{m} \omega^{5}+m \omega^{7}\right) \Rightarrow \frac{\epsilon}{m} \omega^{5}+m \omega^{7}=\frac{d u}{u} .
$$

Consequently,

$$
d \omega^{7}=-\frac{d u}{u} \wedge \omega^{7} \Rightarrow d\left(u \omega^{7}\right)=0
$$

and hence

$$
\omega^{7}=\frac{d v}{u}, \quad \omega^{5}=\frac{\epsilon m(d u-m d v)}{u} .
$$

An ansatz for $\omega^{2}, \omega^{3}, \omega^{4}, \omega^{6}$

We can now determine an ansatz for $\omega^{2}, \omega^{3}, \omega^{4}, \omega^{6}$. Plugging (E.1), into the $d \omega^{1}$ equation, we obtain

$$
d x \wedge d p+d y \wedge d q=\omega^{2} \wedge \omega^{4}+\omega^{3} \wedge \omega^{6} .
$$

Taking the interior product with $\frac{\partial}{\partial u}$, we find that

$$
0=\left(i_{\partial_{u}} \omega^{2}\right) \omega^{4}-\left(i_{\partial_{u}} \omega^{4}\right) \omega^{2}+\left(i_{\partial_{u}} \omega^{3}\right) \omega^{6}-\left(i_{\partial_{u}} \omega^{6}\right) \omega^{3}
$$


and hence $i_{\partial_{u}} \omega^{k}=0$ and similarly $i_{\partial_{v}} \omega^{k}=i_{\partial_{z}} \omega^{k}=0$ for $k=2,3,4,6$. Thus,

$$
\omega^{k}=A^{k} d p+B^{k} d q+C^{k} d x+D^{k} d y
$$

Vranceanu asserts without justification that the coefficients $A^{k}, B^{k}, C^{k}, D^{k}$ can be taken to only depend on $u, v$. In Lemma E.1, we show that we can reduce to dependence on $x, y, u, v$, but it is unclear why a further simplification is a priori necessary.

\section{$\omega^{4}$ and $\omega^{6}$}

We observe from the $d \omega^{4}$ and $d \omega^{6}$ equations, that

$$
d\left(\omega^{4}+m \omega^{6}\right)=-\frac{3}{2}\left(\omega^{4}+m \omega^{6}\right) \wedge \frac{d u}{u} \Rightarrow 0=d\left(u^{-3 / 2}\left(\omega^{4}+m \omega^{6}\right)\right),
$$

and we make the choice of introducing the $x$-coordinate into the coframe via

$$
\omega^{4}+m \omega^{6}=u^{3 / 2} d x .
$$

Now substitute into the $d \omega^{6}$ equation and simplify to get

$$
d\left(\frac{\omega^{6}}{\sqrt{u}}\right)=d(v d x)
$$

and we make the choice of introducing the $y$-coordinate into the coframe via

$$
\omega^{6}=-\sqrt{u}(d y-v d x), \quad \omega^{4}=u^{3 / 2} d x+m \sqrt{u}(d y-v d x) .
$$

\section{$\omega^{2}$ and $\omega^{3}$}

Using the ansatz (E.3), we write

$$
\begin{aligned}
& \omega^{2}=\sigma_{1} \omega^{6}+\mu \omega^{4}+\alpha_{1} d p+\beta_{1} d q, \\
& \omega^{3}=\sigma_{2} \omega^{4}+\rho \omega^{6}+\alpha_{2} d p+\beta_{2} d q .
\end{aligned}
$$

Plugging these and (E.1) into $d \omega^{1}$, we obtain $\sigma_{1}=\sigma_{2}=: \sigma$ and

$$
\begin{aligned}
d x \wedge d p+d y \wedge d q= & -\left(\sqrt{u}(u-m v) \alpha_{1}+v \sqrt{u} \alpha_{2}\right) d x \wedge d p+\sqrt{u}\left(m \alpha_{1}-\alpha_{2}\right) d p \wedge d y \\
& +\left(\sqrt{u}(u-m v) \beta_{1}+v \sqrt{u} \beta_{2}\right) d q \wedge d x-\sqrt{u}\left(m \beta_{1}-\beta_{2}\right) d y \wedge d q
\end{aligned}
$$

which can be solved to obtain

$$
\begin{aligned}
& \omega^{2}=\sigma \omega^{6}+\mu \omega^{4}-u^{-3 / 2}(d p+v d q), \\
& \omega^{3}=\sigma \omega^{4}+\rho \omega^{6}-m u^{-3 / 2}(d p+v d q)+u^{-1 / 2} d q,
\end{aligned}
$$

for some functions $\sigma, \mu, \rho$.

Lemma E.1. $\sigma, \mu, \rho$ only depend on $x, y, u, v$.

Proof. Since $\omega^{4}, \omega^{6}$ depend only on $d x, d y$ and $\omega^{5}, \omega^{7}$ depend only on $d u, d v$, it suffices to show that $d \sigma, d \mu, d \rho$ depend only on $\omega^{4}, \omega^{5}, \omega^{6}, \omega^{7}$. The structure equations (6.5) indicate that $d \omega^{k} \equiv 0 \bmod \left\{\omega^{5}, \omega^{7}\right\}$ for $k=2,3,4,6$. Applying $d$ to (E.4), (E.5), we obtain

$$
\begin{array}{ll}
d \omega^{2} \equiv d \sigma \wedge \omega^{6}+d \mu \wedge \omega^{4} & \bmod \left\{\omega^{5}, \omega^{7}\right\}, \\
d \omega^{3} \equiv d \sigma \wedge \omega^{4}+d \rho \wedge \omega^{6} & \bmod \left\{\omega^{5}, \omega^{7}\right\},
\end{array}
$$


so necessarily,

$$
\begin{array}{ll}
d \sigma \equiv \sigma_{4} \omega^{4}+\sigma_{6} \omega^{6} & \bmod \left\{\omega^{5}, \omega^{7}\right\}, \\
d \mu \equiv \mu_{4} \omega^{4}+\sigma_{4} \omega^{6} & \bmod \left\{\omega^{5}, \omega^{7}\right\}, \\
d \rho \equiv \sigma_{6} \omega^{4}+\rho_{6} \omega^{6} & \bmod \left\{\omega^{5}, \omega^{7}\right\} .
\end{array}
$$

We now make the simplifying search for solutions where $\sigma, \mu, \rho$ are functions of $u, v$ only. Thus,

$$
d \sigma=\frac{\partial \sigma}{\partial \omega^{5}} \omega^{5}+\frac{\partial \sigma}{\partial \omega^{7}} \omega^{7}, \quad d \mu=\frac{\partial \mu}{\partial \omega^{5}} \omega^{5}+\frac{\partial \mu}{\partial \omega^{7}} \omega^{7}, \quad d \rho=\frac{\partial \rho}{\partial \omega^{5}} \omega^{5}+\frac{\partial \rho}{\partial \omega^{7}} \omega^{7} .
$$

Then substitution of (E.4), (E.5) into the structure equations for $\omega^{2}$ and $\omega^{3}$ yields

$$
\begin{aligned}
d \omega^{2}= & \left(\frac{\partial \sigma}{\partial \omega^{5}}+\frac{2 \epsilon \sigma}{m}+\epsilon \mu\right) \omega^{5} \wedge \omega^{6}+\left(\frac{\partial \sigma}{\partial \omega^{7}}+2 m \sigma+\rho\right) \omega^{7} \wedge \omega^{6}+\left(\frac{\partial \mu}{\partial \omega^{5}}+\frac{3 \epsilon \mu}{m}\right) \omega^{5} \wedge \omega^{4} \\
& +\left(\frac{\partial \mu}{\partial \omega^{7}}+2 \sigma+m \mu\right) \omega^{7} \wedge \omega^{4}+\omega^{3} \wedge \omega^{7}-\left(\frac{3 \epsilon}{2 m} \omega^{5}+\frac{m}{2} \omega^{7}\right) \wedge \omega^{2}, \\
d \omega^{3}= & \left(\frac{\partial \sigma}{\partial \omega^{5}}+\frac{2 \epsilon \sigma}{m}+\epsilon \mu\right) \omega^{5} \wedge \omega^{4}+\left(\frac{\partial \sigma}{\partial \omega^{7}}+2 m \sigma+\rho\right) \omega^{7} \wedge \omega^{4} \\
& +\left(\frac{\partial \rho}{\partial \omega^{5}}+2 \epsilon \sigma+\frac{\epsilon \rho}{m}\right) \omega^{5} \wedge \omega^{6}+\left(\frac{\partial \rho}{\partial \omega^{7}}+3 m \rho\right) \omega^{7} \wedge \omega^{6}+\epsilon \omega^{2} \wedge \omega^{5} \\
& -\left(\frac{\epsilon}{2 m} \omega^{5}+\frac{3 m}{2} \omega^{7}\right) \wedge \omega^{3} .
\end{aligned}
$$

Comparison with the structure equations yields

$$
\begin{aligned}
\frac{\partial \mu}{\partial \omega^{5}} & =-\frac{3 \epsilon \mu}{m}-1, & \frac{\partial \sigma}{\partial \omega^{5}} & =-\frac{2 \epsilon \sigma}{m}-\epsilon \mu, & \frac{\partial \rho}{\partial \omega^{5}} & =-2 \epsilon \sigma-\frac{\epsilon}{m} \rho, \\
\frac{\partial \mu}{\partial \omega^{7}} & =-2 \sigma-m \mu, & \frac{\partial \sigma}{\partial \omega^{7}} & =-2 m \sigma-\rho, & \frac{\partial \rho}{\partial \omega^{7}} & =-3 m \rho-1 .
\end{aligned}
$$

For $\gamma^{i}{ }_{j k}$ defined as in (2.8), we have

$$
\left[\frac{\partial}{\partial \omega^{j}}, \frac{\partial}{\partial \omega^{k}}\right]=-\gamma_{j k}^{i} \frac{\partial}{\partial \omega^{i}} \Rightarrow\left[\frac{\partial}{\partial \omega^{5}}, \frac{\partial}{\partial \omega^{7}}\right]=-m \frac{\partial}{\partial \omega^{5}}+\frac{\epsilon}{m} \frac{\partial}{\partial \omega^{7}} .
$$

Using this commutator relation, we can verify that all integrability conditions for the system (E.6), (E.7) are satisfied. Using (E.2), we have for $F=F(u, v)$,

$$
d F=F_{u} d u+F_{v} d v=\frac{u F_{u}}{\epsilon m} \omega^{5}+u\left(m F_{u}+F_{v}\right) \omega^{7},
$$

and so

$$
\frac{\partial F}{\partial \omega^{5}}=\frac{u F_{u}}{\epsilon m}, \quad \frac{\partial F}{\partial \omega^{7}}=\left(m F_{u}+F_{v}\right) u=u F_{v}+\epsilon m^{2} \frac{\partial F}{\partial \omega^{5}} .
$$

We use these expressions for the coframe derivatives to rewrite the system (E.6), (E.7) and obtain expressions for $\sigma, \mu, \rho$. The three equations in (E.6) are respectively equivalent to

$$
\begin{aligned}
& \left(u^{3} \mu\right)_{u}=-\epsilon m u^{2} \quad \Rightarrow \quad \mu=-\frac{\epsilon m}{3}+\frac{a_{1}(v)}{u^{3}} \\
& \left(u^{2} \sigma\right)_{u}=-m u \mu \quad \Rightarrow \quad \sigma=\frac{\epsilon m^{2}}{6}+\frac{m a_{1}(v)}{u^{3}}+\frac{a_{2}(v)}{u^{2}}
\end{aligned}
$$




$$
(u \rho)_{u}=-2 m \sigma \Rightarrow \rho=-\frac{\epsilon m^{3}}{3}+\frac{m^{2} a_{1}(v)}{u^{3}}+\frac{2 m a_{2}(v)}{u^{2}}+\frac{a_{3}(v)}{u} .
$$

Using these expressions for $\sigma, \mu, \rho$, the three equations in (E.7) are respectively equivalent to

$$
a_{1}^{\prime}(v)=-2 a_{2}(v), \quad a_{2}^{\prime}(v)=-a_{3}(v), \quad a_{3}^{\prime}(v)=-\alpha,
$$

where $\alpha=1-\epsilon m^{4}$. The general solution of (E.8) is

$$
a_{3}(v)=-\alpha v+c_{3}, \quad a_{2}(v)=\frac{1}{2} \alpha v^{2}-c_{3} v+c_{2}, \quad a_{1}(v)=-\frac{1}{3} \alpha v^{3}+c_{3} v^{2}-2 c_{2} v+c_{1},
$$

for arbitrary constants $c_{1}, c_{2}, c_{3}$. Taking the simplest choice $c_{1}=c_{2}=c_{3}=0$ yields

$$
\mu=-\frac{\epsilon m}{3}-\frac{\alpha v^{3}}{3 u^{3}}, \quad \sigma=\frac{\epsilon m^{2}}{6}-\frac{m \alpha v^{3}}{3 u^{3}}+\frac{\alpha v^{2}}{2 u^{2}}, \quad \rho=-\frac{\epsilon m^{3}}{3}-\frac{m^{2} \alpha v^{3}}{3 u^{3}}+\frac{m \alpha v^{2}}{u^{2}}-\frac{\alpha v}{u},
$$

and these can be substituted into our ansatz for $\omega^{2}, \omega^{3}$. Finally, our explicit parametrization for the coframe on $\Sigma_{7}$ satisfying the structure equations (6.5) is given by (7.1).

\section{F Calculation of the nine-dimensional symmetry algebras}

We provide here the calculation of the (contact) symmetries of the maximally symmetric generic hyperbolic PDE's $F=0$ corresponding to the normal forms (7.4) and (7.5). A direct approach by computing one of:

1) internal symmetries of $I_{F}$,

2) external symmetries of $F$,

3) symmetries of the lifted coframe on $\Sigma_{7} \times H$,

without any prior simplifications is highly impractical owing to the complexity of the equation. We first make several observations which allow us to simplify the calculation dramatically.

Any equation of the form $F(r, s, t)=0$ admits a six-parameter family of symmetries

$$
\tilde{x}=c_{1}+c_{6} x, \quad \tilde{y}=c_{2}+c_{6} y, \quad \tilde{z}=c_{3}+c_{4} x+c_{5} y+c_{6}{ }^{2} z
$$

corresponding to the symmetry generators

$$
\frac{\partial}{\partial x}, \quad \frac{\partial}{\partial y}, \quad \frac{\partial}{\partial z}, \quad x \frac{\partial}{\partial z}, \quad y \frac{\partial}{\partial z}, \quad x \frac{\partial}{\partial x}+y \frac{\partial}{\partial y}+2 z \frac{\partial}{\partial z} .
$$

These are clearly symmetries because the induced action on the second derivative coordinates $r$, $s, t$ is trivial. (Equivalently, the prolongations of the vector fields (F.1) to $J^{2}\left(\mathbb{R}^{2}, \mathbb{R}\right.$ ) have no components along $\partial_{r}, \partial_{s}, \partial_{t}$.)

We will treat both cases (7.4) and (7.5) simultaneously by using the parametric form of the equation. Using (7.3), we have the following vector fields tangent to the equation manifold in $J^{2}\left(\mathbb{R}^{2}, \mathbb{R}\right)$ :

$$
\begin{aligned}
& \frac{\partial}{\partial w}=-\epsilon m w^{2} \frac{\partial}{\partial r}-\epsilon m^{2} w \frac{\partial}{\partial s}-\epsilon m^{3} \frac{\partial}{\partial t}, \\
& \frac{\partial}{\partial v}=-v^{2} \frac{\partial}{\partial r}+v \frac{\partial}{\partial s}-\frac{\partial}{\partial t} .
\end{aligned}
$$

The key is noticing the following $C^{\infty}\left(\Sigma_{7}\right)$-linear combinations of $\frac{\partial}{\partial w}, \frac{\partial}{\partial v}$ that can be expressed purely in terms of the 2 -jet variables $x, y, z, p, q, r, s, t$ :

$$
w \frac{\partial}{\partial w}+v \frac{\partial}{\partial v}=3 r \frac{\partial}{\partial r}+2 s \frac{\partial}{\partial s}+t \frac{\partial}{\partial t},
$$




$$
\begin{aligned}
& m \frac{\partial}{\partial w}-\frac{\partial}{\partial v}=2 s \frac{\partial}{\partial r}+t \frac{\partial}{\partial s}+\alpha \frac{\partial}{\partial t}, \\
& (m y+w x) \frac{\partial}{\partial w}-(y-v x) \frac{\partial}{\partial v}=(2 y s+3 x r) \frac{\partial}{\partial r}+(y t+2 x s) \frac{\partial}{\partial s}+(\alpha y+x t) \frac{\partial}{\partial t} .
\end{aligned}
$$

These three vector fields are also tangent to the equation manifold, but they are clearly not contact vector fields since: (1) they do not preserve the contact ideal, and (2) they do not arise as prolongations of vector fields on $J^{1}\left(\mathbb{R}^{2}, \mathbb{R}\right)$ (c.f. Bäcklund's theorem). However, this leads us to the following problem: Do (F.2)-(F.4) describe the $r, s, t$ components of contact vector fields on $J^{2}\left(\mathbb{R}^{2}, \mathbb{R}\right)$ ? If so, then those contact vector fields would also be tangent to the equation manifold and hence would correspond to contact symmetries of the equation. The search is greatly simplified by the observation that the components in (F.2)-(F.4) are linear in $x, y, r$, $s, t$ and independent of $z, p, q$.

By Bäcklund's theorem, any contact vector field on $J^{2}\left(\mathbb{R}^{2}, \mathbb{R}\right)$ is the prolongation of a contact vector field on $J^{1}\left(\mathbb{R}^{2}, \mathbb{R}\right)$. Hence, we look at a generalized vector field of order one on $J^{0}\left(\mathbb{R}^{2}, \mathbb{R}\right)$

$$
X=\xi^{1}(x, y, z, p, q) \frac{\partial}{\partial x}+\xi^{2}(x, y, z, p, q) \frac{\partial}{\partial y}+\eta(x, y, z, p, q) \frac{\partial}{\partial z}
$$

and examine its prolongation $X^{(2)}=p r^{(2)}(X)$ on $J^{2}\left(\mathbb{R}^{2}, \mathbb{R}\right)$. If we write

$$
X^{(2)}=X+\eta^{x} \frac{\partial}{\partial p}+\eta^{y} \frac{\partial}{\partial q}+\eta^{x x} \frac{\partial}{\partial r}+\eta^{x y} \frac{\partial}{\partial s}+\eta^{y y} \frac{\partial}{\partial t},
$$

then the standard prolongation formula [22] is

$$
\eta^{J, i}=D_{i} \eta^{J}-\left(D_{i} \xi^{j}\right) z_{J, j},
$$

where $D_{i}$ are total derivative operators and we have used the notation $x^{1}=x, x^{2}=y . J$ is an unordered (symmetric) multi-index, so that for example $z_{1}=p, z_{12}=z_{21}=s$, etc. Ian Anderson's JetCalculus package in Maple v.11 was very useful for computing and manipulating these prolongations. We give an outline of the calculation here.

In order that the components of $X^{(2)}$ in the directions $\frac{\partial}{\partial r}, \frac{\partial}{\partial s}, \frac{\partial}{\partial t}$, have: (1) no dependence on third-order terms, and (2) no quadratic dependence on $r, s, t$, we must have (using (F.5)),

$$
\begin{aligned}
& 0=\eta_{p}-p\left(\xi^{1}\right)_{p}-q\left(\xi^{2}\right)_{p} \\
& 0=\eta_{q}-p\left(\xi^{1}\right)_{q}-q\left(\xi^{2}\right)_{q} \\
& 0=\eta_{p p}-p\left(\xi^{1}\right)_{p p}-q\left(\xi^{2}\right)_{p p}-2\left(\xi^{1}\right)_{p} \\
& 0=\eta_{q q}-p\left(\xi^{1}\right)_{q q}-q\left(\xi^{2}\right)_{q q}-2\left(\xi^{2}\right)_{q} \\
& 0=\eta_{p q}-p\left(\xi^{1}\right)_{p q}-q\left(\xi^{2}\right)_{p q}-\left(\xi^{1}\right)_{q}-\left(\xi^{2}\right)_{p} .
\end{aligned}
$$

Differentiating (F.6), (F.7) with respect to $p$ or $q$ and comparing with (F.8)-(F.10) leads to the conclusion that $\xi^{1}, \xi^{2}, \eta$ are all independent of $p, q$. Consequently, the vector fields we derive are necessarily infinitesimal point transformations, i.e. they project to vector fields on $J^{0}\left(\mathbb{R}^{2}, \mathbb{R}\right)$.

We first examine the coefficient of $\frac{\partial}{\partial s}$ in $X^{(2)}$, or equivalently $\mathcal{L}_{X^{(2)}}(s)$. Using (F.5),

$$
\begin{aligned}
\mathcal{L}_{X^{(2)}}(s)= & -p^{2} q\left(\xi^{1}\right)_{z z}-p q^{2}\left(\xi^{2}\right)_{z z}-p^{2}\left(\xi^{1}\right)_{y z}-2 p s\left(\xi^{1}\right)_{z}-p t\left(\xi^{2}\right)_{z}-q^{2}\left(\xi^{2}\right)_{x z} \\
& -q r\left(\xi^{1}\right)_{z}-2 q s\left(\xi^{2}\right)_{z}+\left(\eta_{z z}-\left(\xi^{1}\right)_{x z}-\left(\xi^{2}\right)_{y z}\right) p q-r\left(\xi^{1}\right)_{y}-t\left(\xi^{2}\right)_{x} \\
& +\left(\eta_{y z}-\left(\xi^{1}\right)_{x y}\right) p+\left(\eta_{x z}-\left(\xi^{2}\right)_{x y}\right) q+\left(\eta_{z}-\left(\xi^{1}\right)_{x}-\left(\xi^{2}\right)_{y}\right) s+\eta_{x y} .
\end{aligned}
$$

Referring back to (F.2)-(F.4), we require $\mathcal{L}_{X^{(2)}}(s) \in \operatorname{span}_{\mathbb{R}}\{s, t, y t, x s\}$. From (F.11), we must have $\xi^{1}=\xi^{1}(x), \xi^{2}=\xi^{2}(x, y)$, and $\eta$ is linear in $z$ with $\eta_{y z}=\eta_{x y}=0$. Thus, $\eta=C_{1}(x)+$ $C_{2}(y)+C_{3}(x) z$. Recalculating $X^{(2)}$, we have

$$
\mathcal{L}_{X^{(2)}}(r)=\eta_{x x}+\left(C_{3}(x)-2\left(\xi^{1}\right)_{x}\right) r-2 s\left(\xi^{2}\right)_{x}+\left(2 C_{3}^{\prime}(x)-\left(\xi^{1}\right)_{x x}\right) p-q\left(\xi^{2}\right)_{x x},
$$




$$
\begin{aligned}
& \mathcal{L}_{X^{(2)}}(s)=-t\left(\xi^{2}\right)_{x}+\left(C_{3}^{\prime}(x)-\left(\xi^{2}\right)_{x y}\right) q+\left(C_{3}(x)-\left(\xi^{1}\right)_{x}-\left(\xi^{2}\right)_{y}\right) s, \\
& \mathcal{L}_{X^{(2)}}(t)=C_{2}^{\prime \prime}(y)+\left(C_{3}(x)-2\left(\xi^{2}\right)_{y}\right) t-\left(\xi^{2}\right)_{y y} q .
\end{aligned}
$$

From (F.2)-(F.4), we require $\mathcal{L}_{X^{(2)}}(r) \in \operatorname{span}_{\mathbb{R}}\{r, s, x r, y s\}$ and $\mathcal{L}_{X^{(2)}}(t) \in \operatorname{span}_{\mathbb{R}}\{1, t, y, t x\}$. Thus, $\eta_{x x}=\left(\xi^{2}\right)_{x x}=\left(\xi^{2}\right)_{y y}=0, C_{2}(y)$ is at most cubic in $y,\left(\xi^{1}\right)_{x}$ is linear in $x$, and $C_{3}^{\prime}(x)=$ $\left(\xi^{2}\right)_{x y}$. Referring to (F.1), we have six symmetries with trivial action on $r, s, t$ and so without loss of generality we can require $\xi^{1}$ to have no constant or linear term, $\xi^{2}$ to have no constant term, and $\xi^{3}$ to have no constant term or terms purely linear in $x, y$. Thus,

$$
\xi^{1}=a_{2} x^{2}, \quad \xi^{2}=b_{01} x+b_{10} y+a_{2} x y, \quad \eta=c_{020} y^{2}+c_{030} y^{3}+c_{001} z+a_{2} x z,
$$

and we have

$$
\begin{aligned}
& \mathcal{L}_{X^{(2)}}(r)=\left(-3 a_{2} x-2 a_{1}+c_{001}\right) r-2\left(b_{01}-a_{2} y\right) s \\
& \mathcal{L}_{X^{(2)}}(s)=\left(-2 a_{2} x-a_{1}-b_{10}+c_{001}\right) s+\left(b_{01}-a_{2} y\right) t \\
& \mathcal{L}_{X^{(2)}}(t)=2 c_{020}+6 c_{030} y+\left(-x a_{2}-2 b_{10}+c_{001}\right) t
\end{aligned}
$$

Comparing (F.12)-(F.14) to each of the desired expressions arising in (F.2)-(F.4), we obtain the remaining symmetry generators

$$
X_{7}=y \frac{\partial}{\partial y}+3 z \frac{\partial}{\partial z}, \quad X_{8}=x \frac{\partial}{\partial y}-\frac{\alpha}{2} y^{2} \frac{\partial}{\partial z}, \quad X_{9}=x^{2} \frac{\partial}{\partial x}+x y \frac{\partial}{\partial y}+\left(x z-\frac{\alpha}{6} y^{3}\right) \frac{\partial}{\partial z} .
$$

\section{Acknowledgements}

It is my pleasure to thank Niky Kamran for his lucid explanations of exterior differential systems and the Cartan equivalence method, his guidance while studying [11], and for bringing to my attention Vranceanu's work [25]. Many of the calculations in this paper were either facilitated by or rechecked using the DifferentialGeometry, LieAlgebras, and JetCalculus packages (in Maple v.11) written by Ian Anderson. I would also like to thank Thomas Ivey and the three anonymous referees for their comments and corrections to help improve the exposition of this article. This work was supported by funding from NSERC and McGill University.

\section{References}

[1] Anderson I.M., Kamran N., Olver P.J., Internal, external, and generalized symmetries, Adv. Math. 100 (1993), 53-100.

[2] Biesecker M., Geometric studies in hyperbolic systems in the plane, Ph.D. Thesis, Utah State University, 2003.

[3] Bryant R.L., Chern S.S., Gardner R.B., Goldschmidt H.L., Griffiths, P.A., Exterior differential systems, Mathematical Sciences Research Institute Publications, Vol. 18, Springer-Verlag, New York, 1991.

[4] Bryant R., Griffiths P., Hsu L., Hyperbolic exterior differential systems and their conservation laws. I, Selecta Math. (N.S.) 1 (1995), 21-112.

[5] Bryant R., Griffiths P., Hsu L., Hyperbolic exterior differential systems and their conservation laws. II, Selecta Math. (N.S.) 1 (1995), 265-323.

[6] Cartan E., Les problèmes d'équivalence, Oeuvres complètes, Vol. 2, Gauthier-Villars, Paris, 1955, 1311-1334.

[7] Courant R., Hilbert D., Methods of mathematical physics, Vol. II: Partial differential equations, Interscience Publishers, New York - London, 1962.

[8] Eendebak P.T., Contact structures of partial differential equations, Ph.D. Thesis, Utrecht University, 2006.

[9] Gardner R.B., A differential geometric generalization of characteristics, Comm. Pure Appl. Math. 22 (1969), $597-626$ 
[10] Gardner R.B., The method of equivalence and its applications, CBMS-NSF Regional Conference Series in Applied Mathematics, Vol. 58, Society for Industrial and Applied Mathematics (SIAM), Philadelphia, PA, 1989.

[11] Gardner R.B., Kamran N., Characteristics and the geometry of hyperbolic equations in the plane, J. Differential Equations 104 (1993), 60-116.

[12] Goursat E., Leçons sur l'intégration des équations aux dérivées partielles du second ordre à deux variables indépendantes, Tome II, Paris, Librairie Scientifique: A. Hermann, 1898.

[13] Juráš M., Geometric aspects of second-order scalar hyperbolic partial differential equations in the plane, Ph.D. Thesis, Utah State University, 1997.

[14] Kushner A., Lychagin V., Rubtsov V., Contact geometry and nonlinear differential equations, Cambridge University Press, 2007.

[15] Kruglikov B., Classification of Monge-Ampère equations with two variables, in Geometry and Topology of Caustics - CAUSTICS '98 (Warsaw), Banach Center Publ., Vol. 50, Polish Acad. Sci., Warsaw, 1999, 179-194.

[16] Lepage T., Sur certaines formes différentielles associées aux équations du type de Monge-Ampère provenant du "Calcul des Variations", Bulletin Acad. Bruxelles 15 (1929), 829-848.

[17] Lychagin V.V., Contact geometry and second-order nonlinear differential equations, Russian Math. Surveys 34 (1979), 137-165.

[18] Marvan M., Vinogradov A.M., Yumaguzhin V.A., Differential invariants of generic hyperbolic MongeAmpère equations, Cent. Eur. J. Math. 5 (2007), 105-133, nlin.SI/0604038.

[19] Morimoto T., La géométrie des équations de Monge-Ampère, C. R. Acad. Sci. Paris, Sér. A-B 289 (1979), A25-A28.

[20] Mubarakzjanov G.M., The classification of the real structure of five-dimensional Lie algebras, Izv. Vyš̌. Učebn. Zaved. Matematika (1963), no. 3 (34), 99-106.

[21] Mubarakzjanov G.M., Classification of solvable Lie algebras of sixth order with a non-nilpotent basis element, Izv. Vysš. Učebn. Zaved. Matematika (1963), no. 4 (35), 104-116.

[22] Olver P.J., Equivalence, invariants, and symmetry, Cambridge University Press, Cambridge, 1995.

[23] Petrescu S., Sur les invariants des équations de Goursat, Ann. Sci. Univ. Jassy I 24 (1938), 232-256.

[24] Stormark O., Lie's structural approach to PDE systems, Encyclopedia of Mathematics and its Applications, Vol. 80, Cambridge University Press, Cambridge, 2000.

[25] Vranceanu G., La géométrisation des équations aux dérivées partielles du second ordre, J. Math. Pures Appl. 6 (1937), 361-374.

[26] Vranceanu G., Sur les invariants des équations aux dérivées partielles du second ordre, Bull. Math. Soc. Roumaine Sci. 42 (1940), 91-105. 